NATIONAL LABORATORY

\title{
Embedded Sensors and Controls to Improve Component Performance and Reliability - System Dynamics Modeling and Control System Design
}

September 2013

Prepared by

Alexander M. Melin

Roger Kisner

David L. Fugate

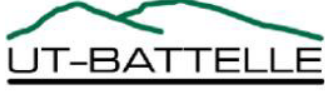




\section{DOCUMENT AVAILABILITY}

Reports produced after January 1, 1996, are generally available free via the U.S. Department of Energy (DOE) Information Bridge:

Web Site: http://www.osti.gov/bridge

Reports produced before January 1, 1996, may be purchased by members of the public from the following source:

National Technical Information Service

5285 Port Royal Road

Springfield, VA 22161

Telephone: 703-605-6000 (1-800-553-6847)

TDD: $703-487-4639$

Fax: 703-605-6900

E-mail: info@ntis.fedworld.gov

Web site: http://www.ntis.gov/support/ordernowabout.htm

Reports are available to DOE employees, DOE contractors, Energy Technology Data Exchange (ETDE), and International Nuclear Information System (INIS) representatives from the following sources:

Office of Scientific and Technical Information

P.O. Box 62

Oak Ridge, TN 37831

Telephone: 865-576-8401

Fax: $865-576-5728$

E-mail: report@osti.gov

Web site:http://www.osti.gov/contact.html

This report was prepared as an account of work sponsored by an agency of the United States Government. Neither the United States nor any agency thereof, nor any of their employees, makes any warranty, express or implied, or assumes any legal liability or responsibility for the accuracy, completeness, or usefulness of any information, apparatus, product, or process disclosed, or represents that its use would not infringe privately owned rights. Reference herein to any specific commercial product, process, or service by trade name, trademark, manufacturer, or otherwise, does not necessarily constitute or imply its endorsement, recommendation, or favoring by the United States Government or any agency thereof. The views and opinions of authors expressed herein do not necessarily state or reflect those of the United States Government or any agency thereof. 


\title{
EMBEDDED SENSORS AND CONTROLS TO IMPROVE COMPONENT PERFORMANCE AND RELIABILITY - SYSTEM DYNAMICS MODELING AND CONTROL SYSTEM DESIGN
}

\author{
Alexander M. Melin \\ Roger Kisner \\ David L. Fugate
}

Date Published: September 2013

\author{
Prepared by \\ OAK RIDGE NATIONAL LABORATORY \\ P.O. Box 2008 \\ Oak Ridge, Tennessee 37831-6285 \\ managed by \\ UT-BATTELLE, LLC \\ for the \\ U.S. DEPARTMENT OF ENERGY \\ under contract DE-AC05-00OR22725
}





\section{Contents}

LIST OF FIGURES $\ldots \ldots \ldots \ldots \ldots \ldots \ldots \ldots \ldots \ldots \ldots \ldots$

LIST OF TABLES $\ldots \ldots \ldots \ldots \ldots \ldots \ldots \ldots \ldots \ldots \ldots$ vii

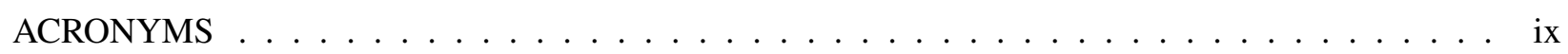

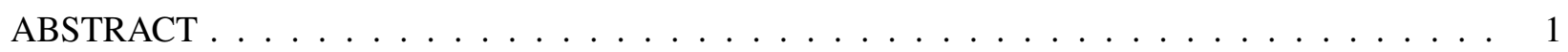

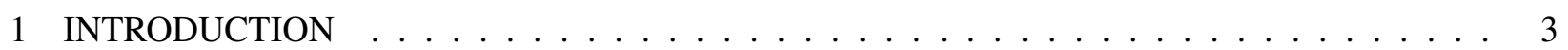

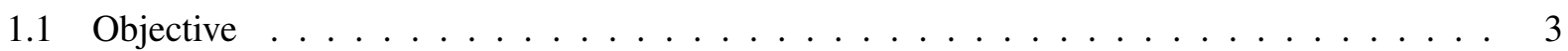

1.2 Overview of Pump Concept $\ldots \ldots \ldots \ldots \ldots \ldots \ldots$

1.3 Overview of Modeling, Simulation, and Control Design . . . . . . . . . . . . 5

2 PUMP SECTION MODELS $\ldots \ldots \ldots \ldots \ldots \ldots \ldots \ldots \ldots$

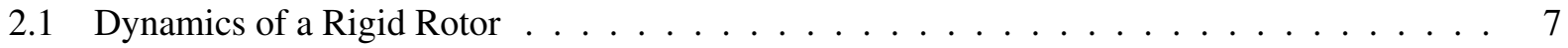

2.1 .1 Equations of Motion $\ldots \ldots \ldots \ldots \ldots$

2.1 .2 Inertial Properties . . . . . . . . . . . . . . . . . . . . 9

2.1 .3 Fluid Effects . . . . . . . . . . . . . . . . . . . . . . 12

2.1 .4 Unforced Response . . . . . . . . . . . . . . . . . . . . . . . . . 16

2.2 Active Magnetic Bearing Model . . . . . . . . . . . . . . . . . 18

2.2 .1 Magnetic Circuit . . . . . . . . . . . . . . . . . . . . . 20

2.2 .2 Linearization . . . . . . . . . . . . . . . . . . . 20

2.2 .3 Operational Boundary Conditions . . . . . . . . . . . . . . . . 21

2.3 Effects of the Rotor and Stator Cans . . . . . . . . . . . . . . . . . . 22

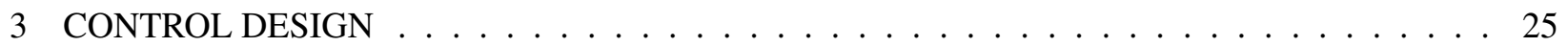

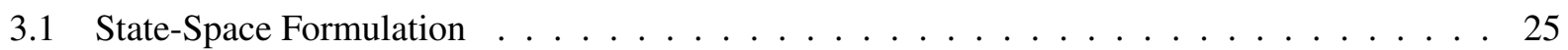

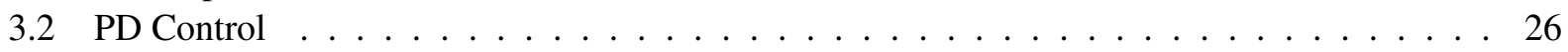

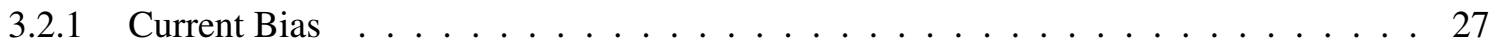

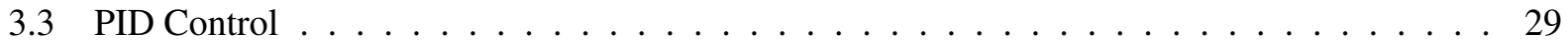

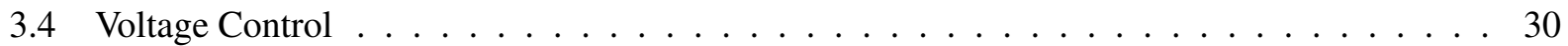

3.5 Linear Quadratic Regulator . . . . . . . . . . . . . . . . . . . . . . 31

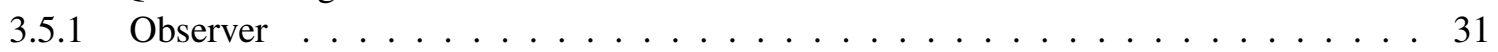

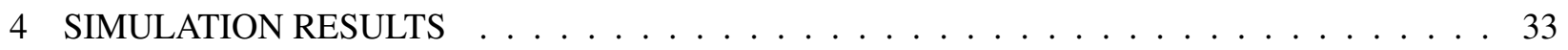

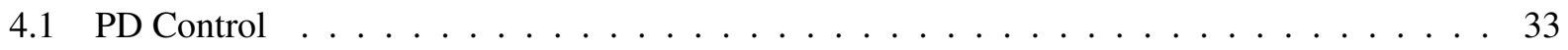

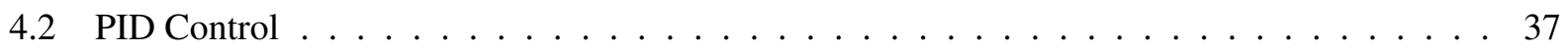

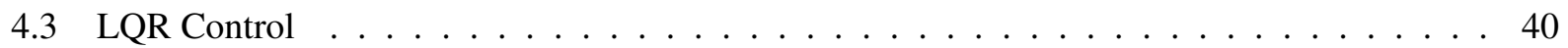

4.4 LQR Control with Integrator $\ldots \ldots \ldots$. . . . . . . . . . . . . . . . 43

5 OPTIMIZATION AND EMBEDDED DESIGN $\ldots \ldots \ldots \ldots$

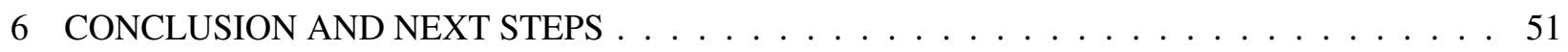

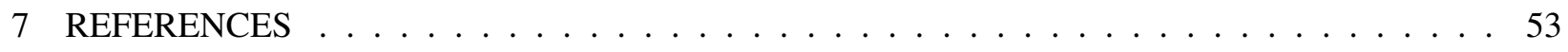





\section{List of Figures}

1 A cross-sectional view of the initial mechanical design of the canned rotor pump with the major systems labeled. . . . . . . . . . . . . . . . . . . 5

2 A cross section of the initial mechanical design of the rotor and associated dimensions in

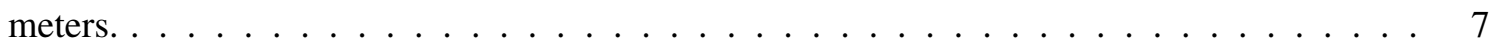

3 The location of the magnetic bearings in relationship to the COM. . . . . . . . . . 9

4 Principal axes corresponding to the principal moments of inertia for the rotor. . . . . . . . 11

5 Dimensionalized stiffness coefficients for the current rotor design. . . . . . . . . . . . . 14

6 Dimensionalized damping coefficients for the current rotor design. . . . . . . . . . . . . . 14

7 The eccentricity ratio as a function of shaft speed in $\mathrm{rad} / \mathrm{s}$. The assumed shaft mass is $25.7539 \mathrm{~kg}$, length is $0.418 \mathrm{~m}$, radius is $0.129 \mathrm{~m}$, and the nominal gap $s_{0}$ is $0.001 \mathrm{~m}$. . . . 15

8 Eccentricity error due to the quantization of the search space used in the numerical method for calculation eccentricity as a function of shaft speed. . . . . . . . . . . . . 16

9 Eigenvalue variation of the unforced rotor system with fluid damping as a function of rotor angular velocity. . . . . . . . . . . . . . . . . . . . . 17

10 Locations of the magnetic laminations for the AMBs and the switched reluctance motor. . . 18

11 Representation of the axial bearing stator configuration and some selected variables. . . . . 19

12 Magnetic properties of Vacodur50 at $750^{\circ} \mathrm{C} \ldots \ldots \ldots \ldots \ldots \ldots$. . . . . . . . . . 22

13 Maximum achievable bandwidth of a Hastelloy $\mathrm{N}$ can as a function of the can thickness. . . 23

14 Closed-loop eigenvalues with a PD controller as a function of rotor speed. Darker points

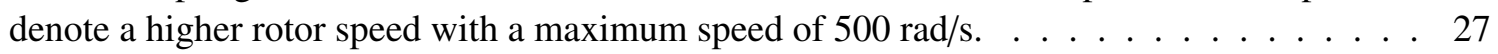

15 Rotor COM movement of the closed-loop system to an impact on the impeller. . . . . . . . . 28

16 Rotor COM angular disturbance to an impact on the impeller using PD control . . . . . . . . 28

17 Variations in the closed-loop eigenvalues for the PD controller as a function of rotor speed. The lighter points denote lower speeds, and conversely, the darker points denote higher rotor speeds. The rotor speed range is $10 \mathrm{rad} / \mathrm{s}$ to $500 \mathrm{rad} / \mathrm{s} \ldots \ldots \ldots$. . . . . . . . . . 33

18 Rotor COM translational disturbance to an impulse impact on the rotor with a PD controller. 34

19 Rotor COM translational movement to a constant disturbance on the rotor with a PD controller. 35

20 Rotor rotational movement about the COM in response to a constant disturbance on the rotor with a PD controller. . . . . . . . . . . . . . . . . . . 35

21 Coil input currents in amps for the AMB denoted by $A$ using a PD controller. Physically, the $A$ bearing is on the opposite end of the shaft from the impeller. . . . . . . . . . . 36

22 Coil input currents in amps for the AMB denoted by $B$ using a PD controller. Physically, the $B$ bearing is on the end of the shaft nearest to the impeller. . . . . . . . . . . . . 36

23 Variations in the closed-loop eigenvalues for the PID controller as a function of rotor speed. The lighter points denote lower speeds, and conversely, the darker points denote higher rotor speeds. The rotor speed range is $10 \mathrm{rad} / \mathrm{s}$ to $500 \mathrm{rad} / \mathrm{s} \ldots \ldots \ldots \ldots \ldots$. . . . . . . . 37

24 Rotor COM translational movement to a constant disturbance on the rotor with a PID con-

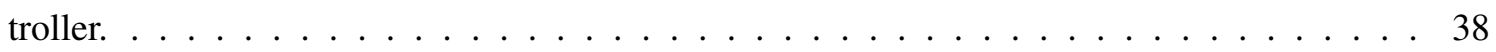

25 Rotor rotational movement about the COM in response to a constant disturbance on the rotor with a PID controller. . . . . . . . . . . . . . . . . . . 38

26 Coil input currents in amps for the AMB denoted by $A$ using a PID controller. Physically, the $A$ bearing is on the opposite end of the shaft from the impeller. . . . . . . . . . . . 39 
27 Coil input currents in amps for the AMB denoted by $B$ using a PID controller. Physically, the $B$ bearing is on the end of the shaft nearest to the impeller. . . . . . . . . . . . . 39

28 The closed-loop eigenvalues for the LQR controller. . . . . . . . . . . . . . . . . . 40

29 Rotor COM translational movement to a constant disturbance on the rotor with an LQR

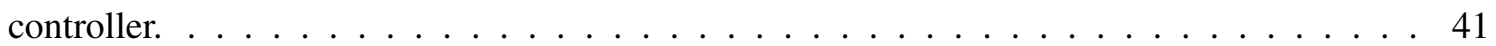

30 Rotor rotational movement about the COM in response to a constant disturbance on the rotor with an LQR controller. . . . . . . . . . . . . . . . . . . . . . . . 41

31 Coil input currents in amps for the AMB denoted by $A$ using an LQR controller. Physically, the $A$ bearing is on the opposite end of the shaft from the impeller. . . . . . . . . . . . . . 42

32 Coil input currents in amps for the AMB denoted by $B$ using an LQR controller. Physically, the $B$ bearing is on the end of the shaft nearest to the impeller. . . . . . . . . . . 42

33 The closed-loop eigenvalues for the LQR controller with an integrator. . . . . . . . . . . . . 43

34 Rotor COM translational movement to a constant disturbance on the rotor with an LQR controller with an integrator. . . . . . . . . . . . . . . . . 44 4

35 Rotor rotational movement about the COM in response to a constant disturbance on the rotor with an LQR controller with an integrator. . . . . . . . . . . . . . . . . . . . . . 44

36 Coil input currents in amps for the AMB denoted by $A$ using an LQR controller with an integrator. Physically, the $A$ bearing is on the opposite end of the shaft from the impeller. . . 45

37 Coil input currents in amps for the AMB denoted by $B$ using an LQR controller with an integrator. Physically, the $B$ bearing is on the end of the shaft nearest to the impeller. . . . . 45

38 Cost function to optimize the magnetic bearing design. None of the boundary conditions are included in this figure . . . . . . . . . . . . . . . . . . . . . 49 


\section{List of Tables}

Page

1 Model parameters used to calculate the fluid stiffness and damping . . . . . . . . . . 13

2 Model parameters used to calculate the fluid stiffness and damping $\ldots \ldots \ldots \ldots$

3 Magnetic parameters for the model . . . . . . . . . . . . . . . . . . . 21 



\title{
ACRONYMS
}

\author{
A/D analog to digital \\ ac alternating current \\ AMB active magnetic bearing \\ AHTR Advanced high-temperature Reactor \\ ASI Advanced Sensors and Instrumentation \\ COM center of mass \\ dc direct current \\ DOF degrees of freedom \\ I\&C instrumentation and controls \\ LQG linear quadratic Gaussian \\ LQR linear quadratic regulator \\ NEET Nuclear Energy Enabling Technologies \\ ODE ordinary differential equation \\ ORNL Oak Ridge National Laboratory \\ PD proportional derivative \\ PID proportional integral derivative \\ PWM pulse width modulation
}





\begin{abstract}
This report documents the current status of the modeling, control design, and embedded control research for the active magnetic bearing (AMB) canned rotor pump being used as a demonstration platform for deeply integrating instrumentation and controls (I\&C) into nuclear power plant components. This pump is a highly interconnected thermo/electro/mechanical system that requires an active control system to operate. Magnetic bearings are an inherently unstable system, and without active, moment-by-moment control, the rotor would contact fixed surfaces in the pump, causing physical damage.

This report details the modeling of the pump rotordynamics, fluid forces, electromagnetic properties of the protective cans, AMBs, power electronics, and interactions between different dynamical models. The system stability of the AMB rotor in both the unforced and controlled configurations is investigated analytically. Additionally, controllers are designed using proportional derivative (PD) control, proportional integral derivative (PID) control, voltage control, and linear quadratic regulator (LQR) control. Finally, a design optimization problem that joins the electrical, mechanical, magnetic, and control system design into one problem to balance the opposing needs of various design criteria using the embedded system approach is presented.
\end{abstract}





\section{INTRODUCTION}

Having embedded sensors and controls in nuclear power plant components is expected to increase their performance and reliability. A transition of component design and functionality from a static mechanical design to a flexible dynamic electromechanical system with embedded sensors and controls can realize components that can adapt in real-time to changing environmental conditions. The device performance can be optimized over a wide range of operating conditions, while the sensors provide diagnostic and prognostic capabilities that can increase component lifetime and reduce operating costs. Design margins of components can be reduced because of tight coupling between sensors, control, and the controller device, resulting for example in lower mass and hence lower costs.

Embedded sensors and controls can also enable features, performance, and reliability that are not possible with legacy approaches. Future nuclear power plant reactor concepts include elevated temperatures and other extreme environmental factors that challenge materials and component designs. For example, some reactor concepts include operating temperatures of around $700^{\circ} \mathrm{C}$ with difficult material compatibility requirements that exclude most materials from use in component design. These environmentally driven constraints on materials result in mechanical designs that are not achievable with current material technology. By deeply embedding sensors and controls into the system, functions that cannot be realized by pure mechanical design approaches can be realized as an actively controlled electro-mechanical system that can now operate in this extreme environment. Of course, sensors that work in this extreme environment are required, without which it would be impossible to develop the feedback control system necessary for functionality of the device.

\subsection{OBJECTIVE}

There are many challenges to developing embedded sensors and controls that can operate in extreme environments. In addition to the new environmental challenges of high-temperatures and material incompatibility, there are the traditional challenges of high-pressure and high-radiation environments. Developing sensors and electrical actuators that can operate in these environments presents many new challenges. For example, high-temperatures exclude standard permanent magnet materials, which reduces options for sensors and electrical actuation. The sealed canned rotor configuration, which is desirable for eliminating rotating seals, drives the requirement for non-contact sensor techniques.

The objective of this report is to present research directly related to the modeling and control design effort performed by Oak Ridge National Laboratory (ORNL) for the Department of Energy's Advanced Sensors and Instrumentation program (ASI), a technology crosscutting initiative under the Nuclear Energy Enabling Technologies (NEET) program. Specific objectives for the embedded I\&C project are as follows.

- Explore and quantify the potential gains from embedded I\&C - improved component reliability, increased performance, and reduced cost.

- Identify practical control, sensing, and measurement techniques for the extreme environments found in high-temperature reactors.

- Design and fabricate a functional prototype high-temperature pump for liquid fluoride salts represents target demonstration of improved performance and reliability and has great potential for widespread usage beyond salt reactor applications. 
By its nature, embedded sensors and controls require tight coupling between their mechanical and electrical systems to derive full advantage of the embedded concept; hence, the embedded concept needs to be studied in the context of a physical device to gain the full benefit of the research. For this demonstration technology, a high-temperature canned rotor pump was chosen. The choice of a reactor coolant pump was made because this system is not only a major source of potential failures, maintenance cost, and downtime for nuclear power plants but also when applied to a high-temperature liquid fluoride salt reactor design it exhibits virtually all of the environmental difficulties that are encountered in the new reactor designs. This makes the technologies for embedded sensors and controls developed under this research program applicable to a wide variety of components beside coolant pumps.

The ORNL report Embedded Sensors and Controls to Improve Component Performance and Reliability: Conceptual Design Report details development of the initial system design requirements, the interactions between the various systems, material considerations, and basic system architecture and develops sensor technologies that are required for operation that can survive in the extreme environment. ${ }^{1}$ Additional information is given in the ORNL report Evaluation of Manufacturability of Embedded Sensors and Controls with Canned Rotor Pump System on the machining, materials, and manufacturing technologies that are suitable for high-temperatures, corrosive environments, and operational stresses. ${ }^{2}$ This document also details assembly issues and the design of the drive electronics.

This technical report documents the results of the modeling, simulation, and control system design research for the canned rotor pump. The results include modeling the rotor dynamics, fluid bearing effects, AMBs, electromagnetic effects of the protective cans, and disturbance forces. The development of a control system similar to those used for industrial AMBs is presented along with the application of some more advanced control system design techniques that are being deployed in actual applications. Finally, optimization is presented using the embedded concept to perform a multidisciplinary design.

\subsection{OVERVIEW OF PUMP CONCEPT}

In this section, a conceptual reactor cooling pump using a canned rotor design is introduced and the operating environment is described. The functional requirement of this cooling pump design is to pump liquid fluoride salt at $700^{\circ} \mathrm{C}$ for reactor cooling. The required power for the demonstration pump is $10 \mathrm{~kW}$. The pump will be prototyped and tested using a high-temperature salt loop at ORNL, which provides a realistic environment. The testing will include the embedded sensors and controls technologies developed during this project and characterization of performance improvements over the existing system.

The liquid fluoride salts are corrosive to most metals that are not nickel based and create problems with shaft seal design and reduce useful lifetime. The use of mechanical bearings in this harsh environment is also problematic. Because of the desire to avoid rotating seals and mechanical bearings, the rotor will be suspended by AMBs and sealed in a protective can. The current design revision is shown in Fig. 1.

The rotor design is based on the following:

- the rotor design consists of a central shaft that provides an attachment to the fluid impeller (mechanical structure),

- the shaft contains two sections of radial bearing lamination components that interact with the stator radial bearing windings (magnetic materials),

- the shaft contains one section of switched reluctance lamination components that interact with the stator motor windings (magnetic materials), 


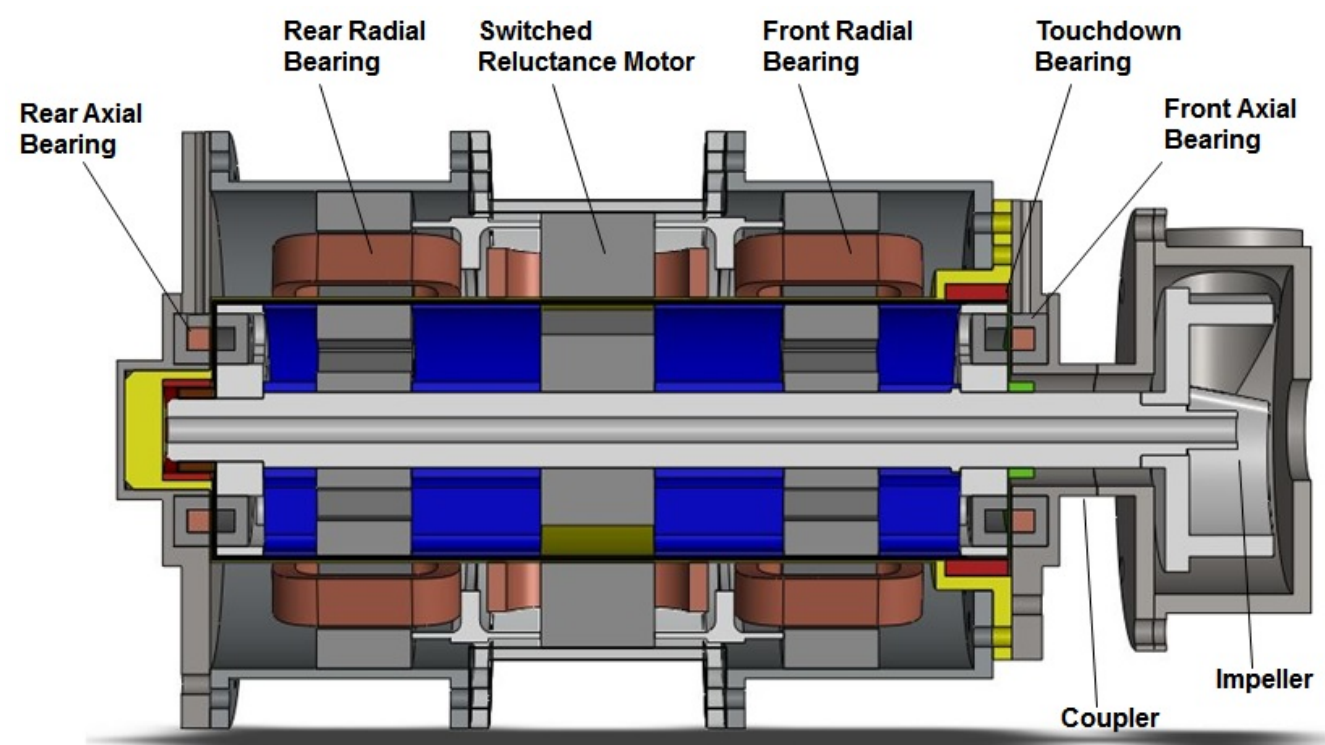

Fig. 1. A cross-sectional view of the initial mechanical design of the canned rotor pump with the major systems labeled.

- the shaft contains various spacer components that provide the proper geometric locations for other shaft components (mechanical structure),

- the shaft contains sections of axial bearing lamination components on each end that interact with stator windings for axial bearing functions (magnetic materials), and

- the entire shaft assembly is sealed in a can to provide a barrier from the molten salt material (mechanical structural).

As an additional safety feature, touch-down bearings will be included in case of a power loss to the controller, although the fluid bearing effects of the liquid fluoride salt between the rotor and stator will provide sufficient rotor stability and damping to prevent any catastrophic damage.

\subsection{OVERVIEW OF MODELING, SIMULATION, AND CONTROL DESIGN}

This report documents the modeling, simulation, and control system design for the rotor AMB suspension system. The sensors and controls are coupled to the mechanical and electrical design and the functionality of the system so that an embedded approach to designing the sensors and controller is required to fully optimize the system.

To aid in the analytical analysis of the magnetic bearing system, the following assumptions are used:

- the switched reluctance motor operation is not directly coupled to the bearing dynamics and can be modeled as an external angular velocity dependent disturbance,

- the impeller forces are not dependent on the rotor position and can be treated as external disturbances,

- the axial magnetic bearing design is functionally identical to the design of the radial magnetic bearings with the exception of being virtually independent of shaft angular movement, and details will not be included in this report for brevity, and 
- the shaft will be considered a rigid body because of the large diameter-to-length ratio and the high shaft material stiffness compared to the effective magnetic bearing stiffness.

These simplifying assumptions allow the development of analytical models of the system which will be used to analyze the behavior over a wide range of operating conditions and parameters. In future work, the fully nonlinear models will be implemented to verify the embedded sensor and control design.

The first step in the development of the embedded sensors and controls is the creation of models describing the dynamic behavior of the subsystems and their interactions. The rotordynamics are described in Sect. 2.1, the fluid bearing effect of the spinning rotor is detailed in Sect. 2.1.3, the dynamics of the AMBs are given in Sect. 2.2, the bandwidth limitations imposed by the rotor and stator cans in the magnetic circuit are given in Sect. 2.3, the combined dynamic model is given in Sect. 3, and the dynamics of the power electronics are described in Sect. 3.4. The switched reluctance motor torque ripple can be treated as an external disturbance on the system that is a function of rotor speed.

Fully parameterized simulations were created in Simulink ${ }^{\complement}$ in both linearized and nonlinear versions. The nonlinear simulation includes continuous nonlinearities from the magnetic properties of the materials in addition to non-continuous nonlinearities arising from contact of the rotor and stator and current limitations.

The development of control systems based on the linearized models is detailed in Sect. 3. An unsophisticated approach to designing a control system for AMBs that is currently used in a large number of industrial AMBs is to use the controller to emulate a spring-damper system. ${ }^{3}$ This has been applied successfully in many applications and has the advantage of being intuitively satisfying. This approach is applied to the canned rotor pump in Sect. 3.2. The addition of an integral term to the controller is outlined in Sect. 3.3. The integral term allows the controller to follow reference commands by the operator and removes the steady state error due to uncertainties in the system parameters. A more advanced control design is presented in Sect. 3.4 that incorporates the power amplifier dynamics into the system model. This allows the controller to use voltage-based actuation instead of current-based actuation. While this requires a more complex control strategy that is not physically intuitive, a voltage controller can be implemented in simpler hardware than a current following power amplifier and take advantage of modern pulse width modulation (PWM) technologies. In Sect. 3.5, an optimal control strategy is applied to the system that utilizes the off-diagonal elements of the control gain matrices to compensate for the cross coupling of the rotordynamics due to gyroscopic effects and fluid bearing effects. This control design provided the best performance of any of the methods investigated. Section 4 compares the response of four control designs, while Sect. 5 outlines the use of the embedded systems concept to perform a holistic multidisciplinary design optimization. Finally, in Sect. 6 conclusions and future work are discussed. 


\section{PUMP SECTION MODELS}

The difficult physical and electrical operating environment of the pump and the reliance on the control system for stable operation necessitate a detailed understanding of the pump behavior over the range of operating conditions. High-fidelity dynamic models of the pump system are useful for understanding the physical behavior and sensing requirements and designing the controller. They will also provide insight into the system time constants, input-output sensitivities, and degree of coupled behavior. These models need to capture the physics of the dynamic behavior of the pump and AMBs during operation and contain parametric features for design optimization and feasibility studies. Because the impeller and switched reluctance motor forces can be decoupled from the motion of the rotor, these forces will be included as an external disturbance and not explicitly modeled. The analysis of the axial bearings is functionally similar to the analysis of the radial bearings with the simplification that the axial bearings do not contend with the rotordynamic forces. For brevity, the analysis of the axial bearings will not be explicitly included. In this section, models are developed for the rigid body motion of the rotor, the electromagnetics of the AMBs, and the fluid forces acting on the rotor. Finally, the interaction between the different dynamical systems is analyzed.

\subsection{DYNAMICS OF A RIGID ROTOR}

In this subsection, the rigid rotor dynamics of the canned rotor pump system are analyzed. All numerical values used in the specific examples and simulations are based on the current system design concept. The rotor dimensions are depicted in Fig. 2.

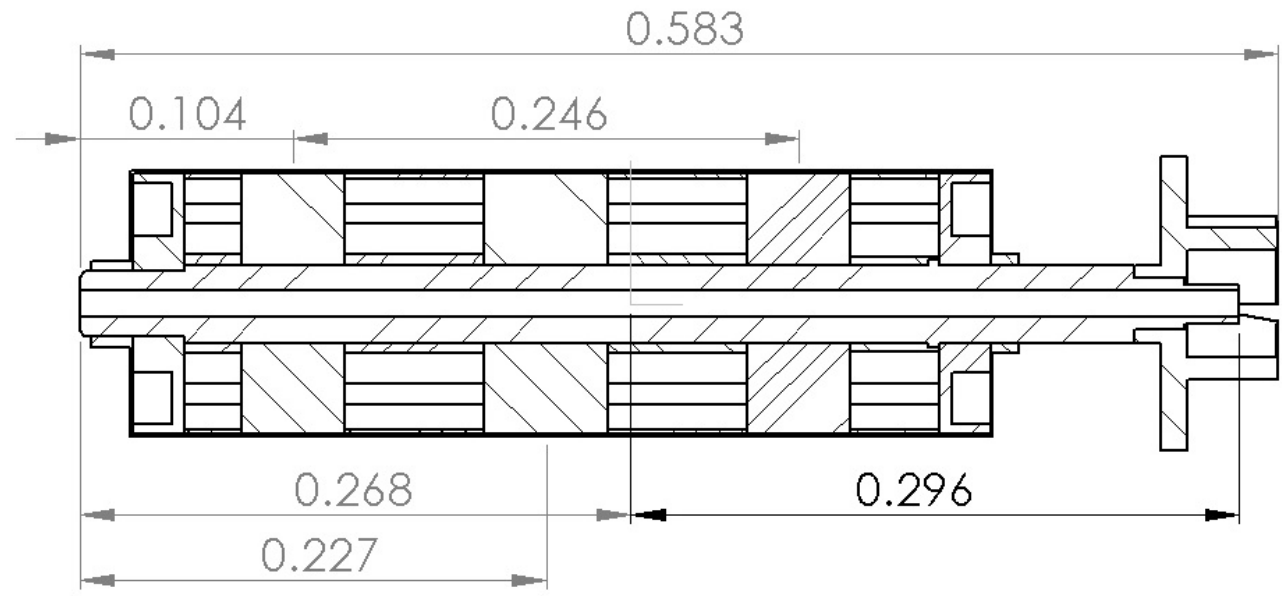

Fig. 2. A cross section of the initial mechanical design of the rotor and associated dimensions in meters.

For the conceptual design, the rigid body model instead of the flexible rotor model will be used. The diameter to length ratio of the conceptual rotor coupled with the high flexural stiffness of the rotor compared to the effective stiffness of the AMBs make the effects of rotor flexure minimal compared to the rotordynamics. For other AMB systems with low diameter-to-length ratios, this assumption is no longer 
valid, and the first flexible modes of the rotor have frequencies near or below the operating frequency of the rotor and can no longer be ignored. The rotordynamics model used is the standard Euclidean rotation and translation model about the center of mass (COM) using lumped mass and inertia parameters.

\subsubsection{Equations of Motion}

The rigid rotor equations of motion will be implemented in a 4 degree of freedom (DOF) system. The 4 DOF model only includes axial translation and rotation and assumes a constant rotor speed $\Omega$. The rotor position is given by Euclidean coordinates $z=\left[x, y, \theta_{x}, \theta_{y}\right]$ for the rotor COM, where $x$ and $y$ are the two radial translations and $\theta_{x}$ and $\theta_{y}$ are the rotations around the $x$ and $y$ axes, respectively. The equation of motion is given by

$$
M \ddot{z}+\left(G+C_{b}\right) \dot{z}+\left(K+K_{b}\right) z=u(t),
$$

where $M$ is the symmetric mass/inertia matrix, $G$ is the skew-symmetric gyroscopic matrix, $C_{b}=C_{b}^{T} \geq 0$ is the symmetric positive definite damping matrix, $K$ is the symmetric stiffness matrix, $K_{b}=-K_{b}^{T}$ is the nonconservative stiffness matrix, and $u(t)$ are the external forces including the AMB suspension forces. The damping term $C_{b}$ and the stiffness term $K_{b}$ are due to fluid film effects between the rotor and the stator. These matrices will be calculated in Sect. 2.1.3. Finally, most rotational machinery utilizes physical bearings that have a stiffness matrix $K$ due to the bearing material properties; for AMBs, the matrix $K=0$ because there is no physical contact between the rotor and a stationary mechanical bearing. To maintain stable operation, the stiffness matrix $K$ has to be implemented virtually by the control system. This shows how the embedded instrumentation and control is fundamentally linked to the dynamic behavior of the system.

Based on the structure of the Euclidean position tensor $x(t)$, the lumped parameter mass/inertia matrix is defined as

$$
M=\left[\begin{array}{cccc}
m & 0 & 0 & 0 \\
0 & m & 0 & 0 \\
0 & 0 & I_{x x} & 0 \\
0 & 0 & 0 & I_{y y}
\end{array}\right],
$$

where $m$ is the mass of the rotor and $I_{x x}$ and $I_{y y}$ are the rotational inertias about the $x$ axis and $y$ axis, respectively. This formulation of the mass/inertia matrix assumes that the rotor has no imbalances and that the physical and inertial axes are perfectly aligned. In the case when imbalances exist, specifics of the necessary modifications to the mass/inertia matrix are given in Sect. 2.1.2.

The conical modes of the rotor motion are due to coupling between the $x$-axis and $y$-axis motion due to gyroscopic effects. This coupling is captured in the gyroscopic matrix given by

$$
G=\left[\begin{array}{cccc}
0 & 0 & 0 & 0 \\
0 & 0 & 0 & 0 \\
0 & 0 & 0 & I_{z z} \omega \\
0 & 0 & -I_{z z} \omega & 0
\end{array}\right],
$$

where $I_{z} z$ is the rotational inertia around the axis of rotation of the rotor and $\omega$ is the angular velocity of the rotor in $\mathrm{rad} / \mathrm{s}$.

While defining the rotor movement and position at the COM simplifies the specification of the equations of motion, the AMBs operate away from the COM. The coordinates of the rotor centerline located at the AMBs are given by $z_{b}=\left[x_{A}, y_{A}, x_{B}, y_{B}\right]^{T}$. Defining a coordinate transform from the COM to $\mathrm{AMB}$ coordinate system and from the AMB to COM coordinate system allows the AMB forces to be 
expressed in the AMB coordinate system, which simplifies the equation structure. These equations will then be transformed to the COM coordinate system and the AMB forces translated to the equivalent forces and moments about the COM. The nonlinear transformations from the COM and AMB coordinates are given by

$$
\begin{aligned}
& x_{A}=x-L_{1} \sin \left(\theta_{x}\right), \\
& x_{B}=x+L_{2} \sin \left(\theta_{x}\right), \\
& y_{A}=y-L_{1} \sin \left(\theta_{y}\right), \text { and } \\
& y_{B}=y+L_{2} \sin \left(\theta_{y}\right),
\end{aligned}
$$

The small motions of the rotor allow the use of the small angle theorem to linearize the coordinate transformation. This linear transformation matrix $B^{T}$ from the COM coordinates to the bearing reference frame is

$$
B^{T}=\left[\begin{array}{cccc}
1 & 0 & -L_{1} & 0 \\
0 & 1 & 0 & -L_{1} \\
1 & 0 & L_{2} & 0 \\
0 & 1 & 0 & L_{2}
\end{array}\right],
$$

where $L_{1} \geq 0$ and $L_{2} \geq 0$ are shown in Fig. 3 and $(\cdot)^{T}$ denotes a matrix transpose. In matrix form, the linear transformation of the COM to AMB coordinate transform is

$$
z_{b}=B^{T} z
$$

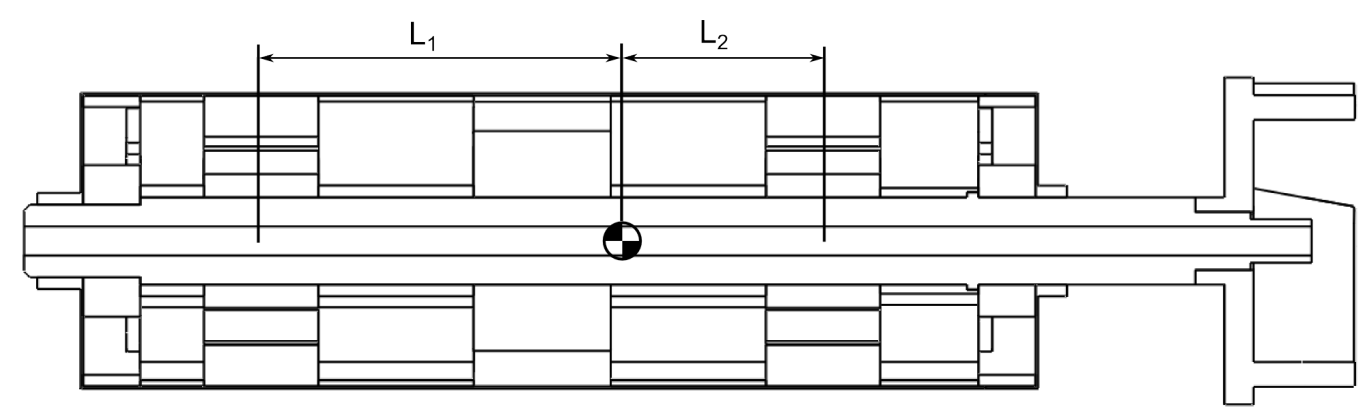

Fig. 3. The location of the magnetic bearings in relationship to the COM.

The transformation from the AMB coordinates to the COM coordinates is given by

$$
z=B z_{b}
$$

\subsubsection{Inertial Properties}

While the simple mass and inertia matrix given in Eg. (2) are sufficient for many situations, the effects of rotor imbalance on the rotordynamics and AMB stability can be large. To study the effects of mass imbalance on the system behavior, a more detailed description of the inertial properties of the rotor and a parameterized description of mass imbalance effects on the mass/inertia matrix are derived. To simplify the development of the inertial properties, the standard method of describing the motion about the COM will be utilized. 
The inertial properties of a rigid body undergoing rotation are described by six mass moments of inertia. In a fixed-body reference frame $P-x y z$ where $P$ is the origin and $[x, y, z]^{T}$ are three mutually orthogonal axes, the inertial properties are given by

$$
\begin{aligned}
I_{x x} & =\int\left(y^{2}+z^{2}\right) \mathrm{d} m, & & I_{y z}=\int y z \mathrm{~d} m, \\
I_{y y} & =\int\left(z^{2}+x^{2}\right) \mathrm{d} m, & & I_{z x}=\int z x \mathrm{~d} m, \text { and } \\
I_{z z} & =\int\left(x^{2}+y^{2}\right) \mathrm{d} m, & I_{x y} & =\int x y \mathrm{~d} m .
\end{aligned}
$$

The inertial properties of the system can be combined into a single symmetric matrix given by

$$
I_{P}=\left[\begin{array}{ccc}
I_{x x} & -I_{x y} & -I_{z x} \\
-I_{x y} & I_{y y} & -I_{y z} \\
-I_{z x} & -I_{y z} & I_{z z}
\end{array}\right]
$$

This inertia matrix can be rotated about the origin $P-x y z$ by a transformation matrix $T$ to a new coordinate system $P^{\prime}-x^{\prime} y^{\prime} z^{\prime}$ where the new inertia matrix is given by

$$
I_{P^{\prime}}=T I_{P} T^{T}
$$

and the axes transformation is given by

$$
[x, y, z]^{T}=T\left[x^{\prime}, y^{\prime}, z^{\prime}\right]^{T} .
$$

It is well understood that there always exists a transformation $T_{0}$ such that the inertia matrix is diagonal and given by

$$
I_{O}=\left[\begin{array}{ccc}
I_{x 0} & 0 & 0 \\
0 & I_{y 0} & 0 \\
0 & 0 & I_{z 0}
\end{array}\right]
$$

and the diagonal elements $I_{x 0}, I_{y 0}, I_{z 0}$ are known as the principal moments of inertia. In the specific case of the rotor shown in Fig. 2 the symmetry of the rotor design allows the principal axes of inertia to be defined as shown in Fig. 4.

Analyzing the conceptual rotor design shown in Fig. 2. The rotor has a mass $M=25.75 \mathrm{~kg}$. The COM is located at $x=0 \mathrm{~m}, y=0 \mathrm{~m}, z=0.268 \mathrm{~m}$. With principal moments of inertia $I_{x}=0.6175 \mathrm{~kg} \cdot \mathrm{m}^{2}$, $I_{y}=0.6175 \mathrm{~kg} \cdot \mathrm{m}^{2}$, and $I_{z}=0.05153 \mathrm{~kg} \cdot \mathrm{m}^{2}$, the inertia matrix for the rotor is

$$
I=\left[\begin{array}{ccc}
I_{x x} & -I_{x y} & -I_{z x} \\
-I_{x y} & I_{y y} & -I_{y z} \\
-I_{z x} & -I_{y z} & I_{z z}
\end{array}\right]=\left[\begin{array}{ccc}
0.6175 & 0 & 0 \\
0 & 0.6175 & 0 \\
0 & 0 & 0.05153
\end{array}\right] \mathrm{kg} \cdot \mathrm{m}^{2}
$$

Imbalances in the rotor will cause the COM to shift slightly, which will cause coupling between the $x$ and $y$ axes during rotation. This can be modeled as a small mass $\Delta m$ attached to the rotor at location $(a, b, c)$. When translating the origin of the body reference frame $P-x y z$ by an amount $(a, b, c)$ to a new origin $S-x y z$, the inertial properties change according to the following

$$
\begin{aligned}
I_{x} & =I_{P x}+m\left(b^{2}+c^{2}\right), & & I_{y z}=I_{P y z}+m b c, \\
I_{y} & =I_{P y}+m\left(c^{2}+a^{2}\right), & & I_{z x}=I_{P z x}+m c a, \text { and } \\
I_{z} & =I_{P z}+m\left(a^{2}+b^{2}\right), & I_{x y} & =I_{P x y}+m a b .
\end{aligned}
$$




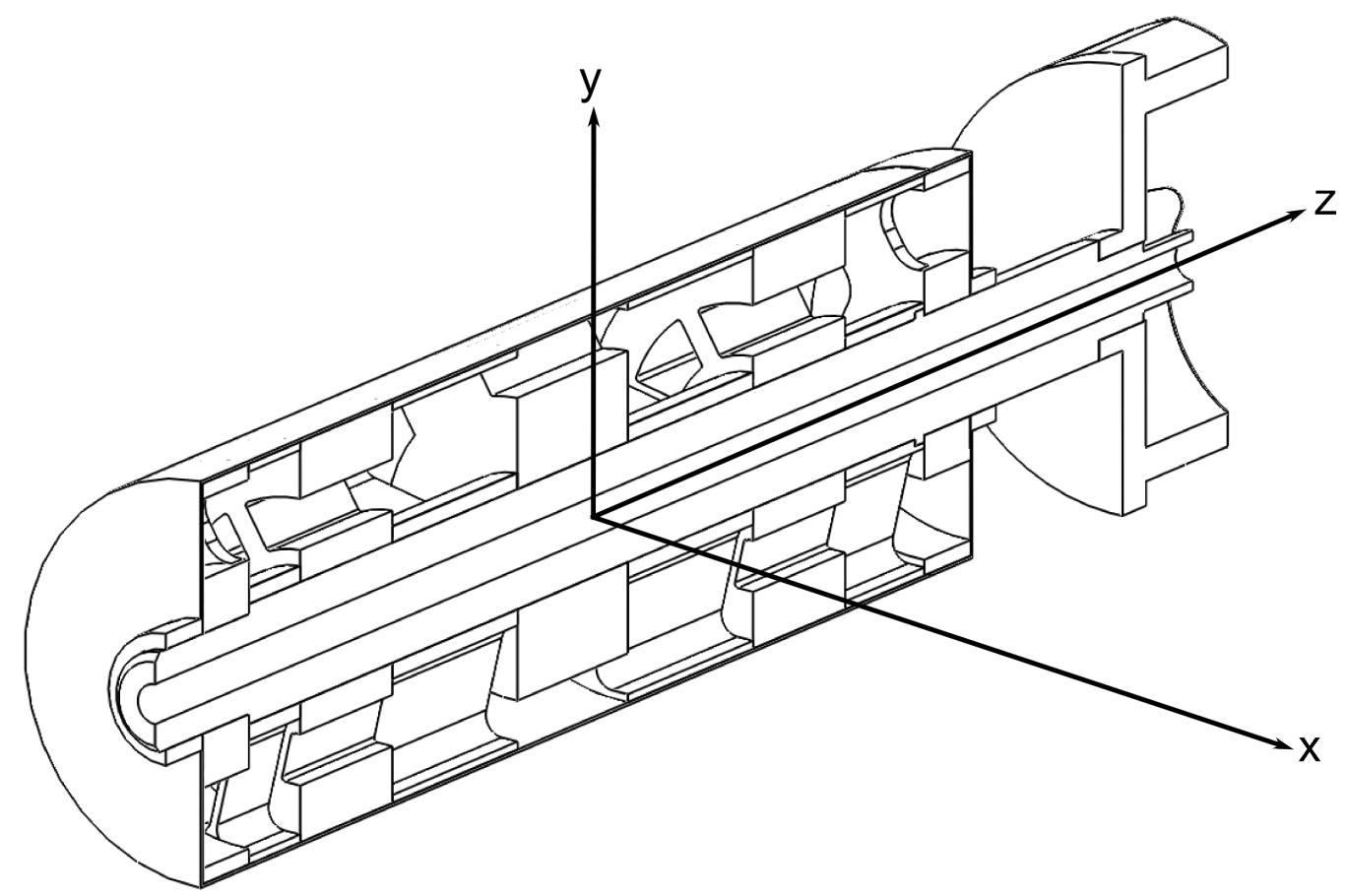

Fig. 4. Principal axes corresponding to the principal moments of inertia for the rotor.

Applying the eccentricity mass $\Delta m$ at the point $(a, b, c)$ and applying Eg. (14) to the principal inertial matrix $I_{O}$ given by Eg. (12) yield the inertial matrix of the disturbed rotor:

$$
I_{S}=I_{O}+\Delta I=\left[\begin{array}{ccc}
I_{x 0} & 0 & 0 \\
0 & I_{y 0} & 0 \\
0 & 0 & I_{z 0}
\end{array}\right]+\left[\begin{array}{ccc}
b^{2}+c^{2} & -a b & -c a \\
-a b & c^{2}+a^{2} & -b c \\
-c a & -b c & a^{2}+b^{2}
\end{array}\right] \Delta m .
$$

Applying this result to the 4 DOF model results in the new mass matrix given by

$$
M=\left[\begin{array}{cccc}
m+\Delta m & 0 & 0 & 0 \\
0 & m+\Delta m & 0 & 0 \\
0 & 0 & I_{x x}+\Delta m\left(b^{2}+c^{2}\right) & -\Delta m(a b) \\
0 & 0 & -\Delta m(a b) & I_{y y}+\Delta m\left(c^{2}+a^{2}\right)
\end{array}\right],
$$

and the new gyroscopic matrix given by

$$
G=\left[\begin{array}{cccc}
0 & 0 & 0 & 0 \\
0 & 0 & 0 & 0 \\
0 & 0 & 0 & \left(I_{z z}+\Delta m\left(a^{2}+b^{2}\right)\right) \omega \\
0 & 0 & -\left(I_{z z}+\Delta m\left(a^{2}+b^{2}\right)\right) \omega & 0
\end{array}\right] .
$$

It can be seen from the cross-coupling terms $-\Delta m(a b)$ in the mass matrix that an imbalance in the rotor will cause a steady state circular motion of the geometric COM.

As an example, from the ISO 1940/1 standard for balance, the maximum permissible unbalance for a balance grade of G100, the maximum allowable unbalance for the conceptual design rotor at $377 \mathrm{rad} / \mathrm{s}$ is $0.715 \mathrm{~g}-\mathrm{mm}$. With a rotor mass of $25.7539 \mathrm{~kg}$, using a mass offset at the location $(0.001,0.001,0) \mathrm{m}$, the corresponding allowable eccentricity mass is $\Delta m=0.506 \mathrm{~g}$. 


\subsubsection{Fluid Effects}

The rotor for the conceptual pump design is fully immersed in the fluid being pumped. The rotor fluid interactions are a major source of external forces on the rotor. Understanding this interaction is important to developing the control system because the fluid interactions will generate cross-coupling rotor instabilities with significant dynamic forces on the rotor.

The first source of fluid force on the rotor arises from the impeller. The impeller design used is identical to the existing impeller used on the high-temperature salt loop at ORNL that will be used to test the prototype system. This impeller is a two-blade design. The impeller forces are independent of the radial movement of the rotor so they will be expressed as an external disturbance force on the shaft end. This disturbance force consists of two components - an axial component that is related to the pump suction head and consequently the rotor angular speed and a radial sinusoidal component due to pressure pulses from the discrete impeller blades.

The second main source of fluid force on the rotor is due to interactions between the liquid fluoride salt and the rotor and stator can. The small fluid-filled gap between the rotor and stator cans acts like a fluid bearing. During normal operation, the rotor and stator geometric centers are aligned by the control system and these fluid forces are purely tangential to the surface of the rotor can due to the velocity differential between the rotor and stator cans. Any small movement of the rotor from geometric center will immediately develop fluid forces on the rotor. These forces are functions of rotor movement and the rotational speed.

The model of the tangential forces on the rotor due to viscous effects from the angular velocity differential is equivalent to the standard model of two large plates moving relative to each other. For large identical flat plates moving relative to each other, the shear force due to fluid viscosity is given by

$$
F=\frac{\mu A v}{y}
$$

where $\mu$ is the fluid viscosity, $A$ is the area of one plate, $v$ is the plate relative velocity of the plates, and $y$ is the distance between the two plates. If the plates are infinite, this is analogous to the cylindrical Couette flow under the assumption that the edge effects are insignificant compared to the surface effects ${ }^{4}$. The cylindrical version of Eg. (18) is given by

$$
T=\left(\frac{2 \pi \mu r^{3} L}{s_{0}}\right) \dot{\theta}_{z}=d_{z} \dot{\theta}_{z}
$$

where $r$ is the radius of the rotor, $L$ is the length of the rotor, $s_{0}$ is the nominal gap between the rotor and stator, and $\dot{\theta}_{z}$ is the axial rotational velocity in $\mathrm{rad} / \mathrm{s} .{ }^{5}$ The torque given in equation 19 only describes the resistance to rotation; to determine the effect of transverse rotor motion, a different approach is needed.

Under dynamic loading conditions, the rotor and stator will not remain concentric which leads to changes in the rotational fluid damping and the addition of damping in the $x$ and $y$ directions. The forces due to the dynamic motion of the rotor about the origin due to fluid bearing effects is given by

$$
\left[\begin{array}{l}
F_{x} \\
F_{y}
\end{array}\right]=\left[\begin{array}{ll}
C_{11} & C_{12} \\
C_{21} & C_{22}
\end{array}\right]\left[\begin{array}{l}
\dot{x} \\
\dot{y}
\end{array}\right]+\left[\begin{array}{ll}
K_{11} & K_{12} \\
K_{21} & K_{22}
\end{array}\right]\left[\begin{array}{l}
x \\
y
\end{array}\right] .
$$

The relationships between the dimensionalized coefficients of the stiffness matrix, damping matrix, and non-dimensional stiffness and damping matrices coefficients are given by

$$
K_{i j}=\frac{F_{0}}{s_{0}} K_{i j}^{*}, \quad C_{i j}=\frac{F_{0}}{\omega s_{0}} C_{i j}^{*}, \quad i, j=1,2,
$$


where $s_{0}$ is the nominal gap between the rotor and stator cans and

$$
F_{0}=F_{\mu} S^{*}=\frac{\mu L^{3} \omega r_{i}}{2 s_{0}^{2}} S^{*}=\frac{\mu L^{3} \omega r_{i}}{2 s_{0}^{2}} \frac{\varepsilon \sqrt{\pi^{2}-\pi^{2} \varepsilon^{2}+16 \varepsilon^{2}}}{2\left(1-\varepsilon^{2}\right)^{2}},
$$

is the static load at a given deflection $e=\varepsilon s_{0}=\sqrt{x^{2}+y^{2}}$, where $0 \leq \varepsilon \leq 1$ is the eccentricity ratio. ${ }^{6}$

The values of the non-dimensional stiffness matrix are given by ${ }^{6}$

$$
\begin{aligned}
& K_{11}^{*}=\left[2 \pi^{2}+\left(16-\pi^{2}\right) \varepsilon^{2}\right] \Psi(\varepsilon), \\
& K_{12}^{*}=\frac{\pi}{4}\left(\frac{\pi^{2}-2 \pi^{2} \varepsilon^{2}-\left(16-\pi^{2}\right) \varepsilon^{4}}{\varepsilon\left(1-\varepsilon^{2}\right)^{1 / 2}}\right) \Psi(\varepsilon), \\
& K_{21}^{*}=-\frac{\pi}{4}\left(\frac{\pi^{2}+\left(32+\pi^{2}\right) \varepsilon^{2}+\left(32-2 \pi^{2}\right) \varepsilon^{4}}{\varepsilon\left(1-\varepsilon^{2}\right)^{1 / 2}}\right) \Psi(\varepsilon), \text { and } \\
& K_{22}^{*}=\frac{\pi^{2}+\left(32+\pi^{2}\right) \varepsilon^{2}+\left(32-2 \pi^{2}\right) \varepsilon^{4}}{1-\varepsilon^{2}} \Psi(\varepsilon),
\end{aligned}
$$

and the values of the non-dimensional damping matrix are given by ${ }^{6}$

$$
\begin{aligned}
& C_{11}^{*}=\frac{\pi\left(1-\varepsilon^{2}\right)^{1 / 2}}{2 \varepsilon}\left[\pi^{2}+\left(2 \pi^{2}-16\right) \varepsilon^{2}\right] \Psi(\varepsilon), \\
& C_{12}^{*}=C_{21}^{*}=-\left[2 \pi^{2}+\left(4 \pi^{2}-32\right) \varepsilon^{2}\right] \Psi(\varepsilon), \text { and } \\
& C_{22}^{*}=\frac{\pi}{2}\left(\frac{\pi^{2}+\left(48-2 \pi^{2}\right) \varepsilon^{2}+\pi^{2} \varepsilon^{4}}{\varepsilon\left(1-\varepsilon^{2}\right)^{1 / 2}}\right) \Psi(\varepsilon),
\end{aligned}
$$

where

$$
\Psi(\varepsilon)=\frac{4}{\left(\pi^{2}+\left(16-\pi^{2}\right) \varepsilon^{2}\right)^{3 / 2}} .
$$

This analysis in standard journal bearings is utilized at nominal rotor positions of $\varepsilon>0$ due to the constant loading on the bearing. In the case of the canned rotor with AMBs levitating the shaft, the eccentricity ratio $\varepsilon \rightarrow 0$ as $t \rightarrow \infty$. For small perturbations around the origin, the damping is given by $\lim _{\varepsilon \rightarrow \infty} C_{i j}(\varepsilon, \omega)$ and the stiffness by $\lim _{e \rightarrow \infty} K_{i j}(\varepsilon, \omega)$ for $i, j=1,2$.

For the conceptual design, Table 1 gives the relevant physical parameters, the variation in the stiffness

Table 1. Model parameters used to calculate the fluid stiffness and damping

\begin{tabular}{ccc}
\hline Parameter & Value & Units \\
\hline$L$ & 0.418 & meters \\
$r_{i}$ & 0.0645 & meters \\
$\omega$ & 377 & rad/s \\
$s_{0}$ & 0.001 & meters \\
$\mu$ & 0.001 & Pa-s \\
\hline
\end{tabular}

coefficients due to changes in the nominal eccentricity ratio are shown in Fig. 5, and the variation in damping coefficients due to changes in the nominal eccentricity ratio are shown in Fig. 6. 


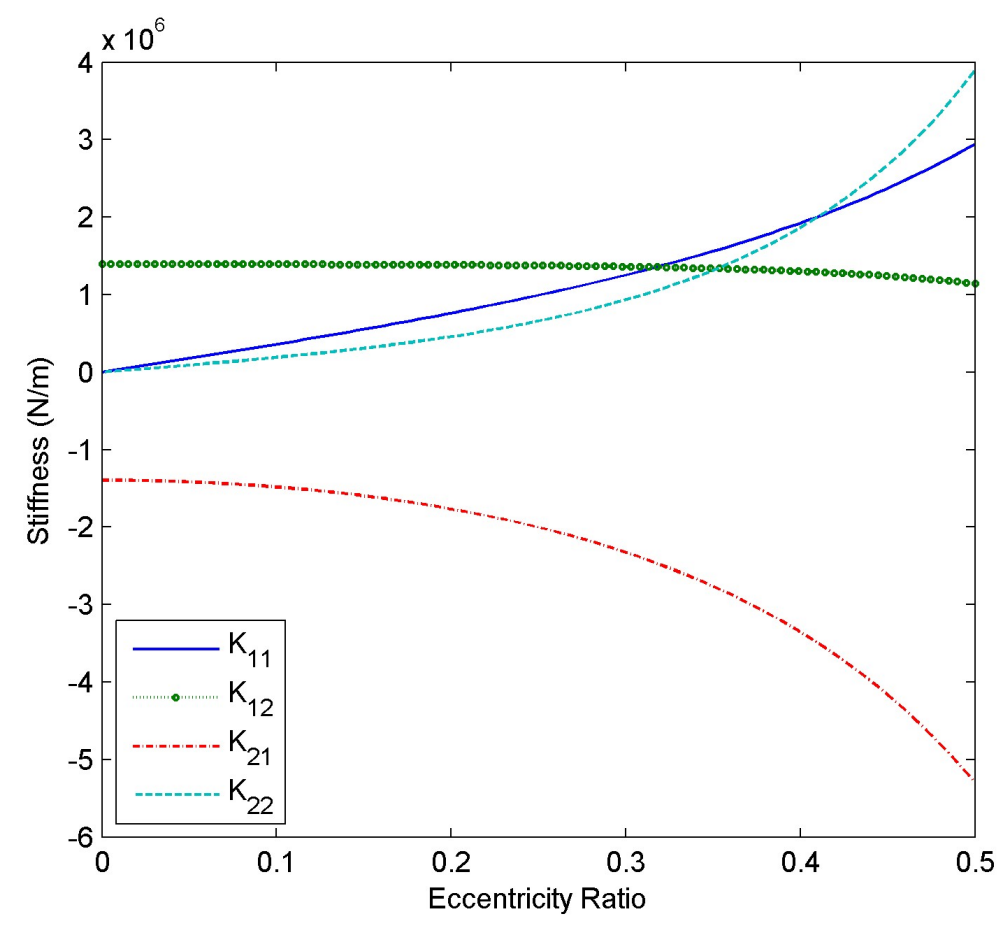

Fig. 5. Dimensionalized stiffness coefficients for the current rotor design.

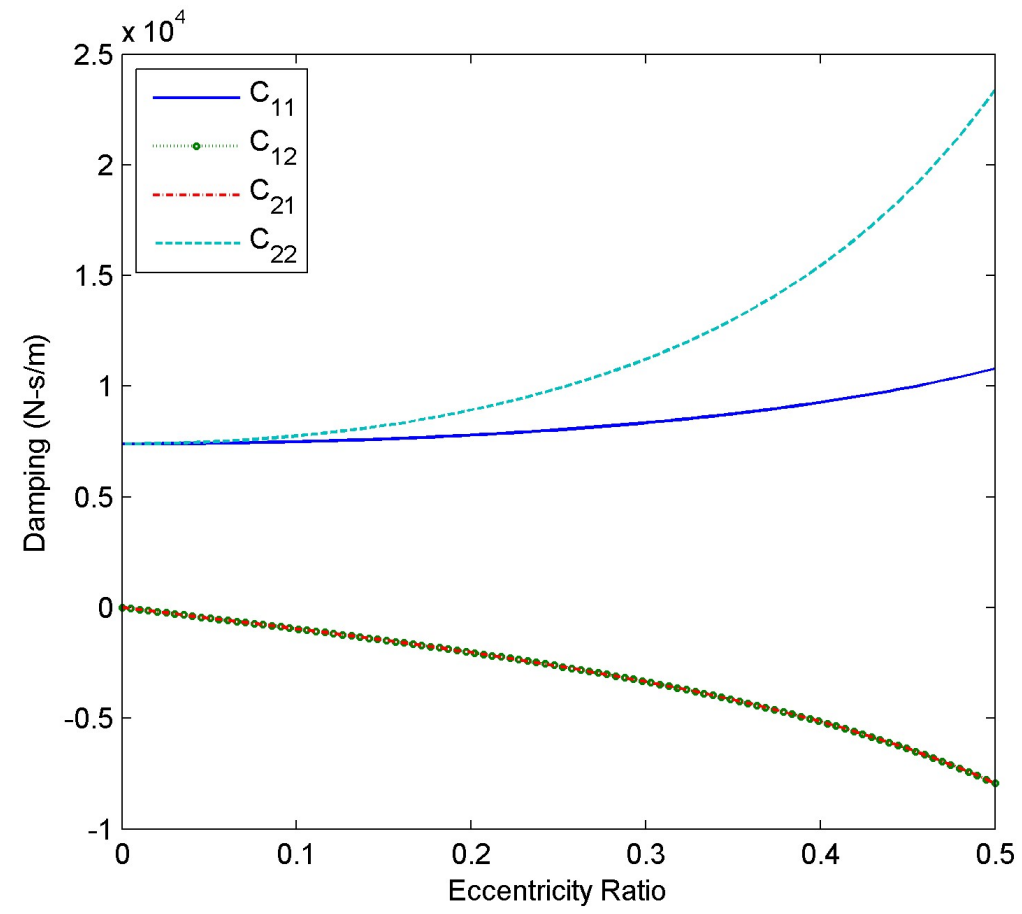

Fig. 6. Dimensionalized damping coefficients for the current rotor design. 
For small movements of the rotor about the origin when the rotor angular velocity $\omega$ is $377 \mathrm{rad} / \mathrm{s}$, the nonconservative fluid stiffness matrix in $\mathrm{N} / \mathrm{m}$ for the current design is

$$
\left[\begin{array}{ll}
K_{11} & K_{12} \\
K_{21} & K_{22}
\end{array}\right]=\left[\begin{array}{cc}
0 & 1.395 \times 10^{6} \\
-1.395 \times 10^{6} & 0
\end{array}\right]
$$

Likewise, the fluid damping matrix in $\mathrm{N}-\mathrm{s} / \mathrm{m}$ is

$$
\left[\begin{array}{ll}
C_{11} & C_{12} \\
C_{21} & C_{22}
\end{array}\right]=\left[\begin{array}{cc}
7,400 & 0 \\
0 & 7,400
\end{array}\right] .
$$

During startup, the rotation will cause a vertical force on the shaft that counteracts the gravitational force. Equation 22 can be used to calculate these forces. A closed-form solution for the eccentricity ratio as a function of the shaft speed does not exist, so numerical methods will be used to calculate the shaft position as a function of rotational speed. As Fig. 7 shows, the rotor moves closer to the geometric center as the shaft speed increases. The numerical error in the calculation is shown in Fig. 8.

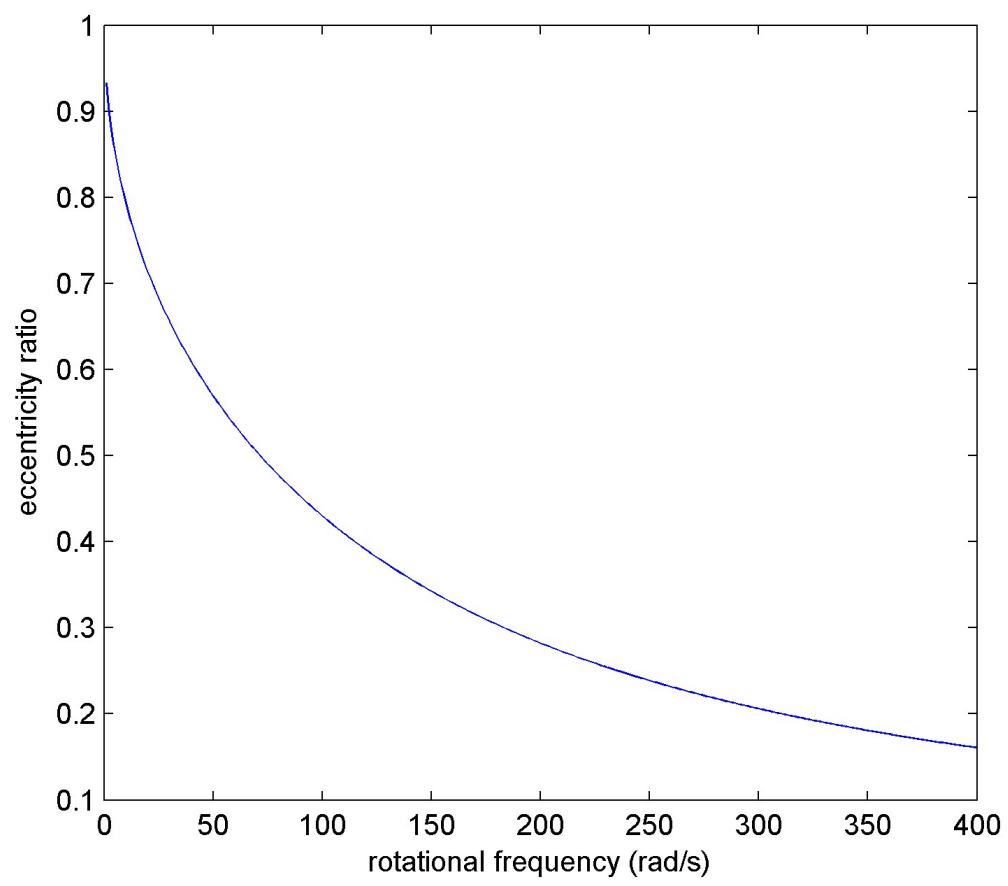

Fig. 7. The eccentricity ratio as a function of shaft speed in rad/s. The assumed shaft mass is $25.7539 \mathrm{~kg}$, length is $0.418 \mathrm{~m}$, radius is $0.129 \mathrm{~m}$, and the nominal gap $s_{0}$ is $0.001 \mathrm{~m}$.

Note that the fluid stiffness and damping matrices only assume radial motion of the rotor and do not take into account rotation of the rotor axis about the COM. This type of motion is expected in the conceptual design. The effect of rotor axis rotational movement can be approximated by applying the fluid stiffness and damping matrices in the AMB coordinate system and transforming the matrices to the COM 


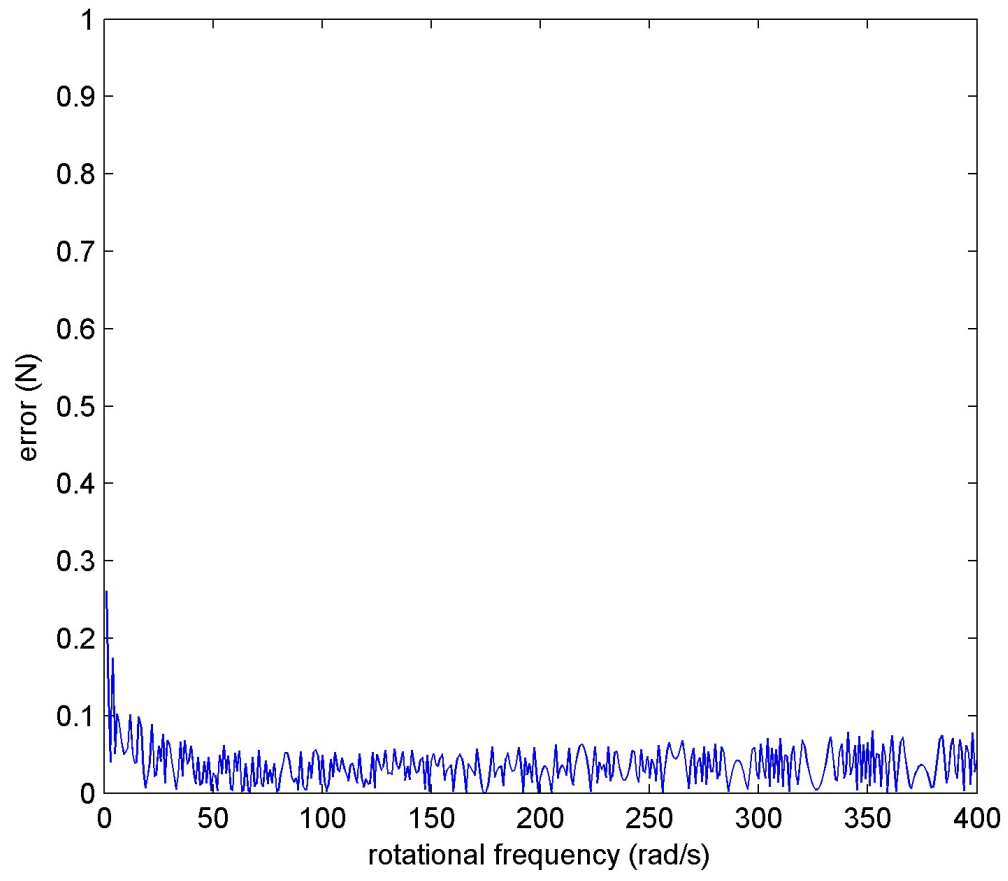

Fig. 8. Eccentricity error due to the quantization of the search space used in the numerical method for calculation eccentricity as a function of shaft speed.

coordinate system. The fluid force equations in the AMB coordinate system are

$$
\begin{aligned}
{\left[\begin{array}{c}
F_{x A} \\
F_{y A} \\
F_{x B} \\
F_{y B}
\end{array}\right] } & =\left[\begin{array}{cccc}
K_{11} & K_{12} & 0 & 0 \\
K_{21} & K_{22} & 0 & 0 \\
0 & 0 & K_{11} & K_{12} \\
0 & 0 & K_{21} & K_{22}
\end{array}\right]\left[\begin{array}{c}
x_{A} \\
y_{A} \\
x_{B} \\
y_{B}
\end{array}\right]+\left[\begin{array}{cccc}
C_{11} & C_{12} & 0 & 0 \\
C_{21} & C_{22} & 0 & 0 \\
0 & 0 & C_{11} & C_{12} \\
0 & 0 & C_{21} & C_{22}
\end{array}\right]\left[\begin{array}{c}
\dot{x}_{A} \\
\dot{y}_{A} \\
\dot{x}_{B} \\
\dot{y}_{B}
\end{array}\right] \text { and } \\
F_{b} & =K_{b} z_{b}+C_{b} \dot{z}_{b} .
\end{aligned}
$$

This can be transformed to the COM coordinate system by

$$
F=F b B^{T}=K_{b} B^{T} z+C_{b} B^{T} \dot{z} .
$$

\subsubsection{Unforced Response}

In the case of the unforced system, we will analyze the eigenvalues using the parameters from Table 2, which includes the values from Table 1.

Due to the lack of physical bearings, in this case, the stiffness matrix $K$ is identical to zero; however, the fluid bearing effect is still present. Combining Eg. (1),Eg. (2), Eg. (3), Eg. (27), and Eg. (26) calculated at the values given in Table 2 yields the second-order matrix differential equation given by

$$
M \ddot{z}+\left(G+C_{b} B^{T}\right) \dot{z}+K_{b} B^{T} z=0 .
$$

Transforming this into state-space form yields

$$
\dot{z}=A z=\left[\begin{array}{cc}
0 & I \\
-M^{-1} K_{b} B^{T} & -M^{-1}\left(G+C_{b} B^{T}\right)
\end{array}\right] z,
$$


Table 2. Model parameters used to calculate the fluid stiffness and damping

\begin{tabular}{ccc}
\hline Parameter & Value & Units \\
\hline$L$ & 0.418 & meters \\
$r_{i}$ & 0.0645 & meters \\
$m$ & 25.7539 & $\mathrm{~kg}$ \\
\hline$I_{x x}$ & 0.6189 & $\mathrm{~kg} / \mathrm{m}^{2}$ \\
$I_{y y}$ & 0.6189 & $\mathrm{~kg} / \mathrm{m}^{2}$ \\
$I_{z z}$ & 0.0515 & $\mathrm{~kg} / \mathrm{m}^{2}$ \\
\hline$L_{1}$ & 0.1746 & $\mathrm{~m}$ \\
$L_{2}$ & 0.0818 & $\mathrm{~m}$ \\
\hline$\omega$ & 377 & $\mathrm{rad} / \mathrm{s}$ \\
$s_{0}$ & 0.001 & $\mathrm{~meters}$ \\
$\mu$ & 0.001 & Pa-s \\
\hline
\end{tabular}

where $z=\left[x, y, \theta_{x}, \theta_{y}, \dot{x}, \dot{y}, \dot{\theta}_{x}, \dot{\theta}_{y}\right]^{T}$ is the new state vector of the rotor COM. Solution of the characteristic equation $\operatorname{det}(\lambda I-A)=0$ gives the eigenvalues of the physical system, which yields information about its stability characteristics. Note that the gyroscopic matrix $G$ is a function of the rotor speed. The unforced open loop eigenvalues are shown in Fig. 9. The presence of eigenvalues in the right half plane shows that

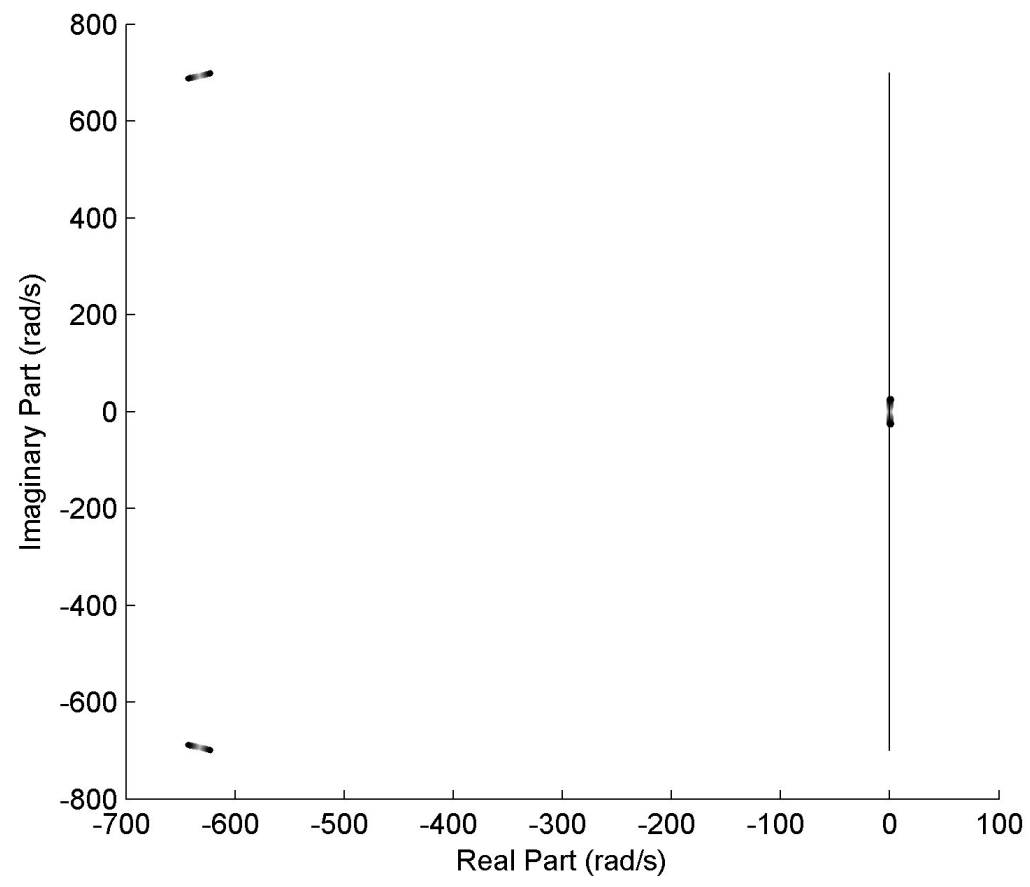

Fig. 9. Eigenvalue variation of the unforced rotor system with fluid damping as a function of rotor angular velocity.

the geometric center for the open loop system is unstable at all rotor speeds. From standard stability 
analysis for fluid bearings, ${ }^{5}$ the rotor stable position in the absence of AMB control will have a non-zero eccentricity and exhibit a limit cycle behavior. This will have an impact on the controllability of the switched reluctance motor and the pump performance but will prevent damage to the pump in the event of loss of control or other catastrophic event.

\subsection{ACTIVE MAGNETIC BEARING MODEL}

The AMBs design chosen to control the radial motion of the rotor consists of two bearings spaced axially on the shaft used to control the radial motion and two bearings at either end of the shaft to stabilize the shaft axially. The axial bearing design is functionally the same as the radial bearing design and will not be expressly stated. The switched reluctance motor will be located in between the two radial magnetic bearings. The locations of the specific components of the AMBs and switched reluctance laminations are shown in Fig. 10 and the configuration of the radial AMBs is shown in Fig. 11.

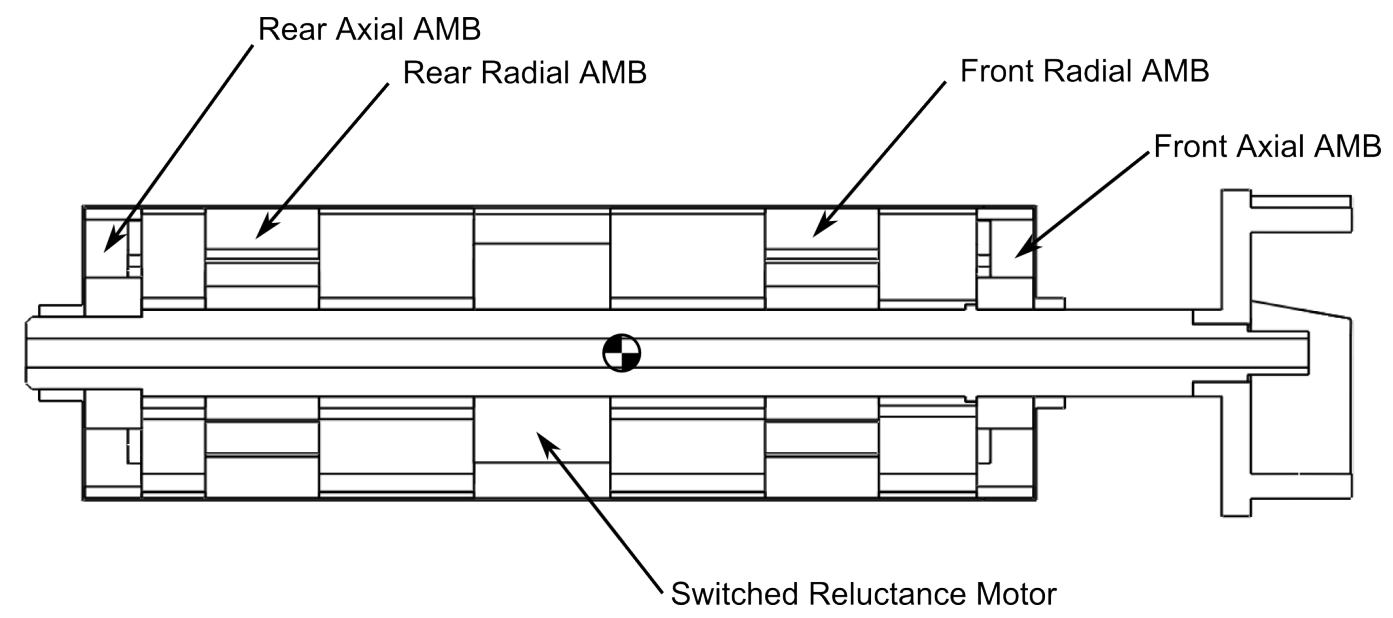

Fig. 10. Locations of the magnetic laminations for the AMBs and the switched reluctance motor.

The pump stator is separated from the rotor laminations by two thin protective metal cans and the molten fluoride salt. While the gap between the rotor and stator does not contain air, the standard terminology in literature for this is an air gap. The air gap in the conceptual design will also be referred to occasionally as a fluid gap.

The following are descriptions and notation for the variables used.

- $N$ - number of turns per double poles

- $A$ - projected surface area of each AMB pole

- $i_{x^{+}}, i_{x^{-}}, i_{y^{+}}, i_{y^{-}}-$coil currents

- $s_{x_{1}^{+}}, s_{x_{2}^{+}}, s_{x_{1}^{-}}, s_{x_{2}^{-}}, s_{y_{1}^{+}}, s_{y_{2}^{+}}, s_{y_{1}^{-}}, s_{y_{2}^{-}}$- air gaps for each individual pole

- $s_{0}$ - the nominal air gap when the rotor is centered

- $(x, y)$ - rotor COM position in the Euclidean coordinate frame

- $(r, \Theta)$ - rotor COM position in the polar coordinate frame 
- $\Theta_{x_{1}^{+}}, \Theta_{x_{2}^{+}}, \ldots, \Theta_{y_{1}^{-}}, \Theta_{y_{2}^{-}}$- the angular position of each pole

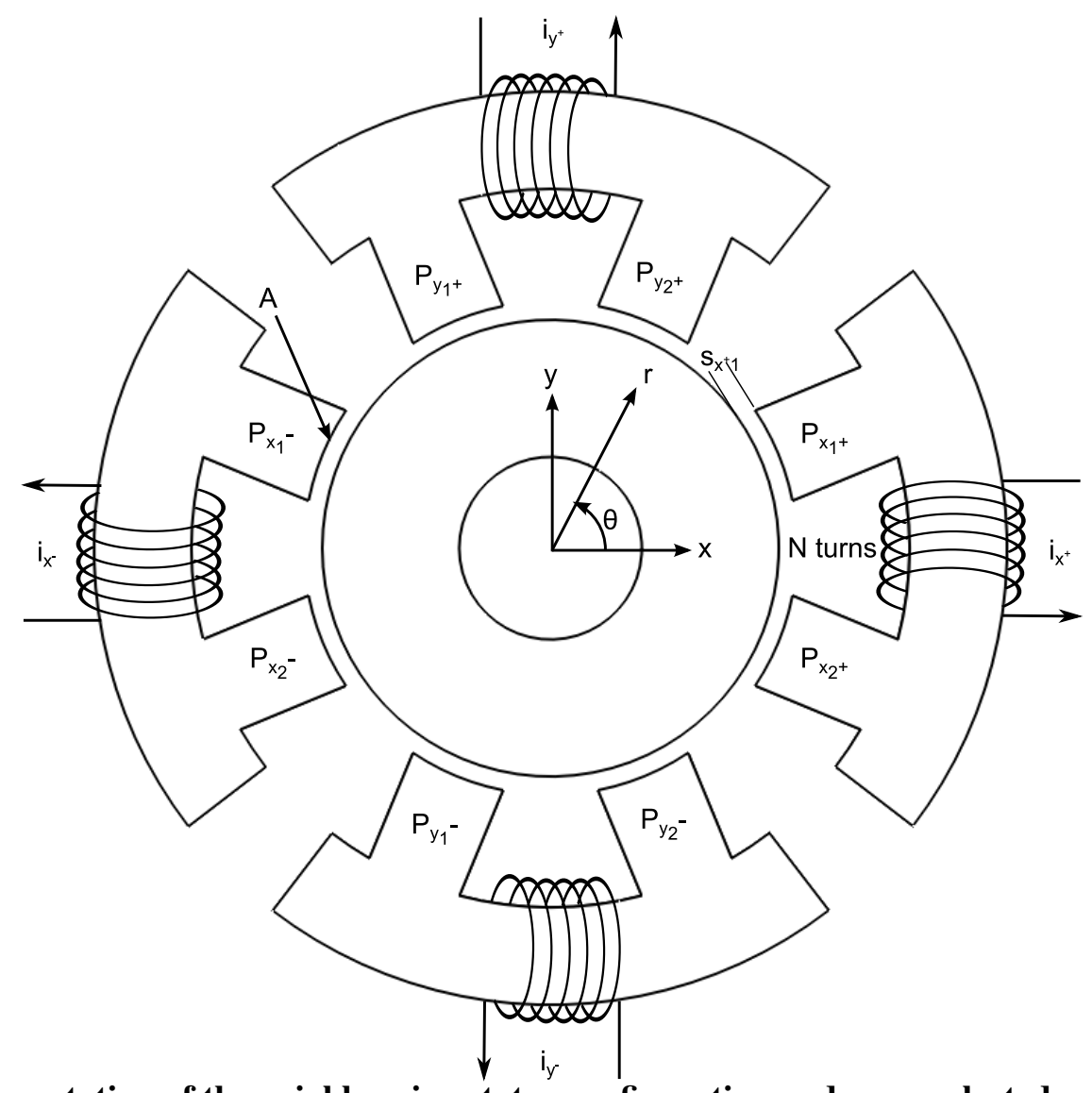

Fig. 11. Representation of the axial bearing stator configuration and some selected variables.

The two axial magnetic bearings will be denoted by superscript $A$ and $B$, respectively. The states of the system are the air gaps, or in the case of the conceptual design, fluid gaps $\left[s_{x^{+}}^{A}, s_{x^{-}}^{A}, s_{y^{+}}^{A}, s_{y^{-}}^{A}, s_{x^{+}}^{B}, s_{x^{-}}^{B}, s_{y^{+}}^{B}, s_{y^{-}}^{B}\right]^{T}$ and the shaft angles $\theta_{x}, \theta_{y}$, and $\theta_{z}$. The control variables are the individual coil currents $\left[i_{x^{+}}^{A}, \ldots, i_{y^{-}}^{A}, i_{x^{+}}^{B}, \ldots, i_{y^{-}}^{B}\right]^{T}$. This does not include any power amplifier dynamics. These will be discussed in Sect. 3.4. The location of the COM of the rotor in relation to the two bearings is shown in Fig. 10. The magnetic force of the bearings is related to the coil current and the distance between the rotor and stator.

To calculate the air gaps in the eight poles we will analyze the system in polar coordinates. The air gap of pole $i$ is given by

$$
s_{i}=s_{0}-r \cos \left(\Theta_{i}-\Theta\right) \text {. }
$$

If the poles are symmetric about the $x$ and $y$ axis, this symmetry can be used to decouple the $s_{x}$ air gaps and the $y$-axis movements. Likewise the $s_{y}$ air gaps and the $x$-axis movement can be decoupled. The decoupled 
equations for the total air gaps for all four axes in a single radial bearing become

$$
\begin{aligned}
& s_{x^{+}}=2\left(s_{0}-x \cos \Theta_{0}\right), \\
& s_{x^{-}}=2\left(s_{0}+x \cos \Theta_{0}\right), \\
& s_{y^{+}}=2\left(s_{0}-y \cos \Theta_{0}\right), \text { and } \\
& s_{y^{-}}=2\left(s_{0}+y \cos \Theta_{0}\right),
\end{aligned}
$$

where $2 \Theta_{0}$ is the angle between two poles. For the configuration shown in Fig. 11, the angle $\Theta_{0}$ is $\pi / 8 \mathrm{rad}$ (22.5 deg).

\subsubsection{Magnetic Circuit}

AMBs use coil windings to develop magnetic flux in the stator magnetic laminations, rotor laminations, and the gap between them forming a magnetic circuit. The flux density $B$ in the magnetic circuit is given by

$$
B=\mu_{0} \frac{N i}{2 s},
$$

where $\mu_{0}=4 \pi \times 10^{-7} \mathrm{~V}$-s/A-m is the magnetic permeability of vacuum, $N$ is the number of coil windings, $i$ is the coil current, and $2 s$ is the total air gap. This calculation of flux density assumes a constant cross-sectional area of the stator, no flux leakage or other losses, and that the magnetic reluctance in the gap between the rotor and stator is much higher than the reluctance of the laminations.

Assuming each of the eight electromagnets on both radial AMBs have coil currents that can be controlled independently, Eg. (35) for a single coil becomes

$$
B_{j}^{k}=\mu_{0} \frac{N i_{j}^{k}}{s_{j}^{k}}, \quad j \in\left\{x^{+}, x^{-}, y^{+}, y^{-}\right\}, \quad k \in\{A, B\},
$$

where $s_{j}^{k}$ are given by Eg. (34).

The force generated by the each magnetic bearing axis is given by

$$
F_{b}=\frac{B^{2} A}{\mu_{0}}
$$

where $B$ is the magnetic flux given by Eg. (36) and $A$ is the cross-sectional area of a magnetic bearing stator tooth. Combining Eg. (36) and Eg. (37) yields

$$
F_{j}^{k}=\frac{1}{4} \mu_{0} N^{2} A \frac{\left(i_{j}^{k}\right)^{2}}{\left(s_{j}^{k}\right)^{2}} \cos \Theta_{0}=k \frac{\left(i_{j}^{k}\right)^{2}}{\left(s_{j}^{k}\right)^{2}} \cos \Theta_{0}, \quad j \in\left\{x^{+}, x^{-}, y^{+}, y^{-}\right\}, \quad k \in\{A, B\} .
$$

\subsubsection{Linearization}

It is common practice to linearize the magnetic bearing force $\mathrm{Eg}$. (38) to aid in the analytical analysis of the bearing behavior and control system design. This is a valid assumption because of the small movement of the shaft; however, care must be taken to validate any controller developed using the linearized model on the nonlinear model to ensure that the unmodeled dynamics do not destabilize the control system. 
By linearizing with respect to both bearing state variables, the linearized force equation for each separate coil can be written as ${ }^{3}$

$$
f(x, i)=k_{i} i-k_{s} x .
$$

The two constants $k_{i}$ and $k_{s}$ are calculated by linearizing Eg. (38) around the operating points $i_{0}$ and $s_{0}$. Assuming that the bearing has rotational symmetry, the constant $k_{i}$ related to the current is given by

$$
k_{i}=\left.\frac{\partial f}{\partial i}\right|_{i=i_{0}, s=s_{0}}=\left.2 k \frac{i}{s^{2}} \cos \Theta_{0}\right|_{i=i_{0}, s=s_{0}} .
$$

Likewise, the spring constant $k_{s}$ related to the rotor movement is given by

$$
k_{s}=\left.\frac{\partial f}{\partial s}\right|_{i=i_{0}, s=s_{0}}=-\left.2 k \frac{i^{2}}{s^{3}} \cos \Theta_{0}\right|_{i=i_{0}, s=s_{0}} .
$$

Table 3 summarizes the magnetic properties of the system design.

Table 3. Magnetic parameters for the model

\begin{tabular}{ccc}
\hline Parameter & Value & Units \\
\hline$\mu_{0}$ & $4 \pi \times 10^{-7}$ & V-s/A-m \\
$N$ & 100 & turns \\
$A$ & 0.0012 & $\mathrm{~m}^{2}$ \\
$i_{0}$ & 30 & $\mathrm{~A}$ \\
$s_{0}$ & 0.001 & $\mathrm{~m}$ \\
\hline$k$ & $3.14159 \times 10^{-9}$ & \\
\hline$k_{i}$ & 0.17415 & \\
$k_{s}$ & -5224.416 & \\
\hline
\end{tabular}

\subsubsection{Operational Boundary Conditions}

During normal operation, the pump will be subjected to various disturbances. These external forces can have a detrimental impact on pump performance and, if sufficiently large, can overload the magnetic bearings and cause physical damage to the pump. Characterizing these extremes in operating condition and designing the AMBs with sufficient performance margins is an important step in analyzing the system.

During startup, the magnetic bearings are required to lift the rotor off of the touchdown bearings. In this case, the distance over which the magnetic bearings must act is twice the nominal air gap. The maximum load capacity of the bearing is related to the air gap, maximum current, and magnetic saturation. Additionally, the maximum current and magnetic saturation are related. The magnetic properties of Vacodur $50,{ }^{7}$ the material chosen to be the rotor magnetic laminations for the magnetic bearings and switched reluctance motor due to the fact that it is practically the only material that has magnetic properties at $700^{\circ} \mathrm{C}$, are shown in Fig. 12. The maximum force that the magnetic bearing can maintain can be derived from Eg. (37) with a flux area of $0.0012 \mathrm{~m}^{2}$ and assuming a magnetic flux saturation at $2.2 \mathrm{~T}$ of $4622 \mathrm{~N}$.

The coil current is physically limited by the conductor ampacity. A practical limit should be chosen based on the coil magnetic flux saturation. In this case the required field strength to achieve a magnetic flux of $2.2 \mathrm{~T}$ is $308 \mathrm{~A} / \mathrm{m}$. This will be the maximum field strength $H_{\max }$ for this design. The maximum field strength can be related to the maximum current and number of turns by

$$
H_{\text {max }} l=N i_{\text {max }}
$$




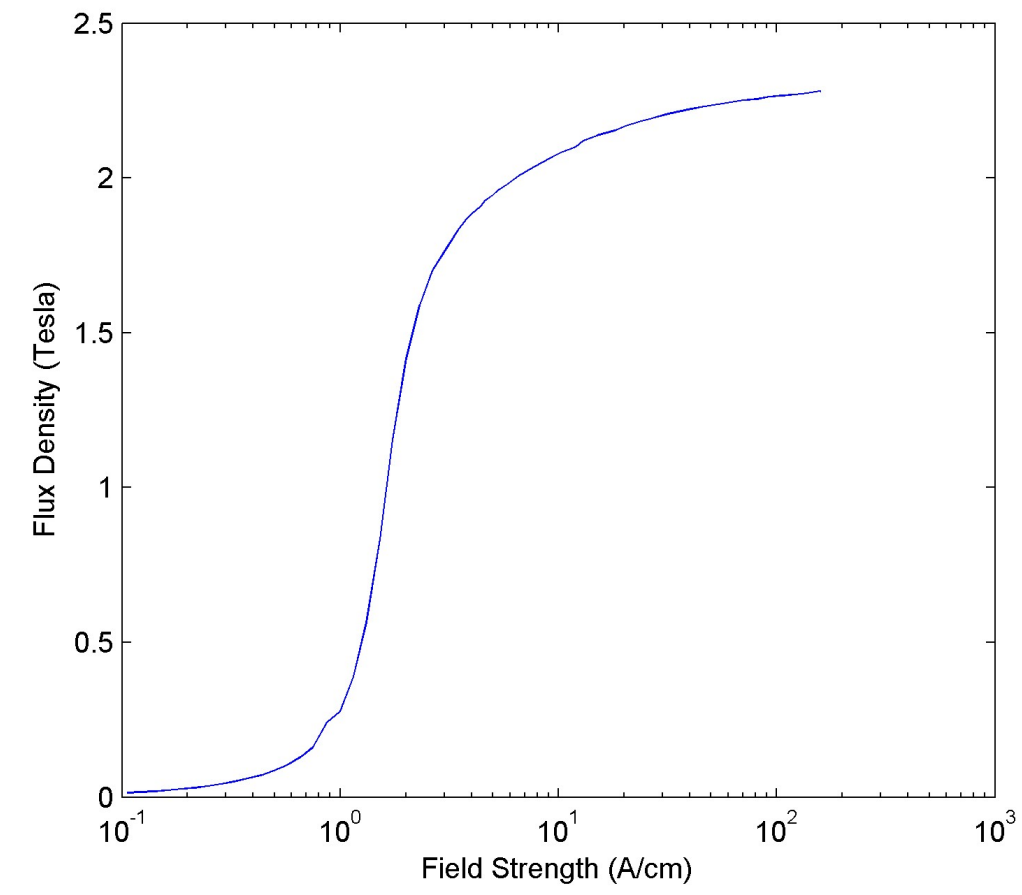

Fig. 12. Magnetic properties of Vacodur50 at $750^{\circ} \mathrm{C}$.

where $l$ is the flux path length through the rotor and stator. Given an approximate path length of the flux in Vacodur 50 of $237 \mathrm{~mm}$, the maximum useful current for $N=100$ turns is $i_{\max }=73 \mathrm{~A}$. The coil currents are also limited by the wire gauge chosen and the number of parallel windings per pole.

\subsection{EFFECTS OF THE ROTOR AND STATOR CANS}

The rotor and stator protective cans are critical to protect the materials in the rotor and stator from the corrosive effects of the liquid fluoride salt. The rotor can will experience potential delamination, growth, creep, and fatique in addition to the corrosive effects experienced by the stator can. The can thickness also has a significant impact on the control system performance. Creation and destruction of eddy currents in the rotor and stator cans due to the time-varying magnetic fields of the AMBs will limit the achievable bandwidth for varying the magnetic field. A reduced bandwidth magnetic field results in reduced bandwidth actuation, which can limit the control system bandwidth. The limitation on the control signal bandwidth will in turn limit the achievable controller gains. Figure 13 shows the maximum achievable bandwidth for a Hastelloy $\mathrm{N}$ can as a function of the can thickness. The frequency response of the can acts like a low pass filter and affects the control system by limiting the maximum controller gains, limiting the achievable loop speed of the controller hardware, and reducing high-frequency signal content in both the actuator signals and the sensor signals. 


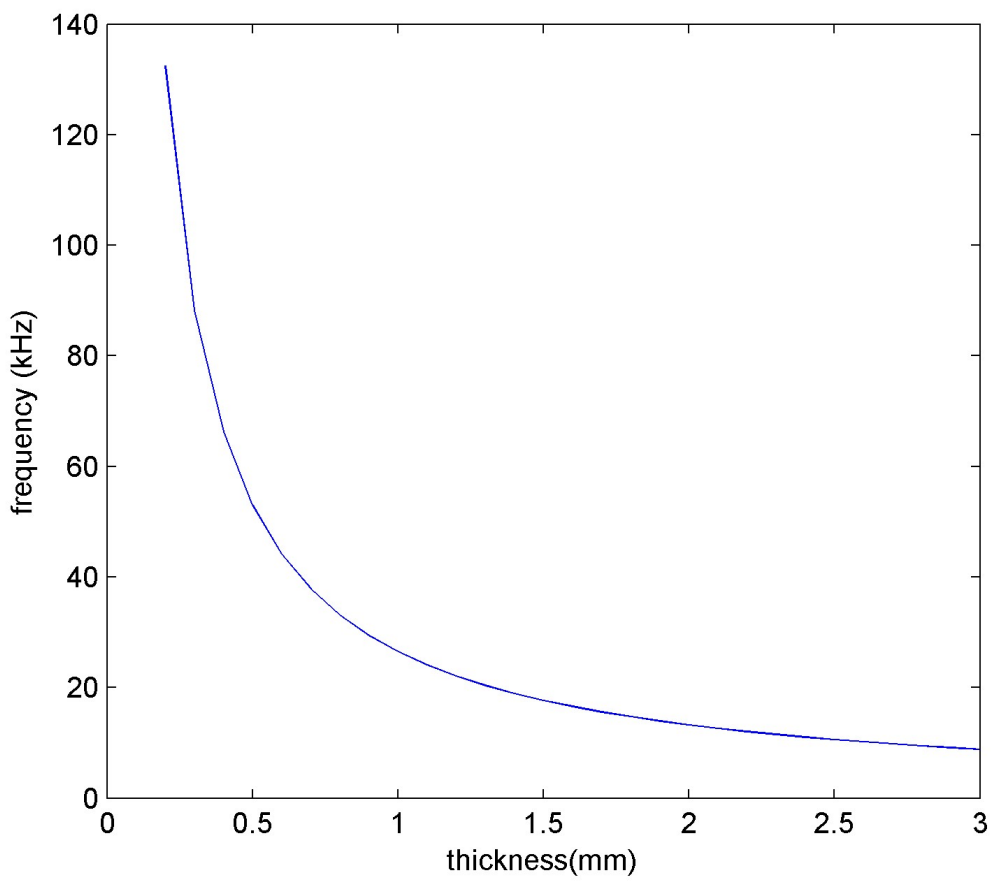

Fig. 13. Maximum achievable bandwidth of a Hastelloy $\mathbf{N}$ can as a function of the can thickness. 



\section{CONTROL DESIGN}

In Sect. 2, models of the pump rotordynamics, fluid forces, and the AMBs were developed. In this section, these models will be used to develop and characterize different control system formulations. The primary single-variable control design technique used for a large number of linear systems is the proportional integral derivative (PID) controller. The next control design technique that will be applied is the linear quadratic regulator (LQR) controller. This technique can improve the system response by taking advantage of the coupling between the orthogonal axes due to gyroscopic effects and fluid rotor interactions. While some simulation results are shown in this section, its primary purpose is to describe the mathematical development of the control system.

The basic system expressed in the COM coordinates system is given by Eg. (43). The rotor position is given by Euclidean coordinates of the $\operatorname{COM} z=\left[x, y, \theta_{x}, \theta_{y}\right]^{T}$, where $x$ and $y$ are the radial translations and $\theta_{x}$ and $\theta_{y}$ are the rotations around the $x$ and $y$ axes, respectively. The equation of motion is given by

$$
M \ddot{z}+\left(G+C_{b} B^{T}\right) \dot{z}+K_{b} B^{T} z=u(t),
$$

where $M$ is the symmetric mass/inertia matrix, $G$ is the skew-symmetric gyroscopic matrix, $C_{b}=C_{b}^{T} \geq 0$ is the damping matrix, $K$ is the symmetric stiffness matrix, $K_{b}=-K_{b}^{T}$ is the nonconservative stiffness matrix, $B^{T}$ is a transformation from the AMB coordinate system to the COM coordinate system, and $u(t)$ are the external forces.

\subsection{STATE-SPACE FORMULATION}

In designing the control system gains, it is desirable to express the AMBs as a linear state-space system. There are two basic coordinate systems that have physical meaning - the coordinates that describe

the movement of the COM of the rotor and the coordinates that describe the actuation of the bearings in the bearing coordinate system. This requires a state change from the COM coordinate system $z=\left[x, y, \theta_{x}, \theta_{y}\right]^{T}$ to the bearing coordinate system $z_{b}=\left[x_{A}, y_{A}, x_{B}, y_{B}\right]^{T}$, where $A$ denotes the axial bearing that is $L_{1} \mathrm{~m}$ away from the COM opposite the impeller and $B$ denotes the axial bearing that is $L_{2} \mathrm{~m}$ from the COM closest to the impeller, as depicted in Fig. 3. The coordinate linearized transformation is given by

$$
z_{b}=B^{T} z=\left[\begin{array}{cccc}
1 & 0 & -L_{1} & 0 \\
0 & 1 & 0 & -L_{1} \\
1 & 0 & L_{2} & 0 \\
0 & 1 & 0 & L_{2}
\end{array}\right]
$$

The input states $u(t)$ are chosen to be the bearing forces $u_{b}=\left[f_{x A}, f_{y A}, f_{x B}, f_{y B}\right]^{T}$. Translating the inputs from the bearing coordinate system $u_{b}(t)$ to the COM coordinate system $u(t)$, the input is given by

$$
u=B u_{b}=\left[\begin{array}{cccc}
1 & 0 & 1 & 0 \\
0 & 1 & 0 & 1 \\
-L_{1} & 0 & L_{2} & 0 \\
0 & -L_{1} & 0 & L_{2}
\end{array}\right]
$$

which is identical to the transpose of $B^{T}$. The linearized bearing derived in Sect. 2.2.2 acting in the AMB coordinate system can be expressed using the rotor COM states $z(t)$ and the coil currents $i(t)$ by

$$
u_{b}(t)=-K_{s} B^{T} z(t)+K_{i} i(t)
$$


where $K_{s}=\operatorname{diag}\left(k_{s}, k_{s}, k_{s}, k_{s}\right), K_{i}=\operatorname{diag}\left(k_{i}, k_{i}, k_{i}, k_{i}\right)$, and $k_{s}$ and $k_{i}$ are given in Table 3 . The bearing forces $u_{b}$ in the AMB coordinate system can in turn be translated to the COM coordinate system using

$$
u(t)=-B K_{s} B^{T} z(t)+B K_{i} i(t) .
$$

Finally, after the appropriate transformations are applied, the second-order linearized ordinary differential equation (ODE) in COM coordinates is given by

$$
M \ddot{z}(t)+\left(G+C_{b} B^{T}\right) \dot{z}(t)+\left(K_{b} B^{T}+B K_{s} B^{T}\right) z(t)=B K_{i} i(t) .
$$

Transforming Eg. (48) into a state-space formulations yields

$$
\begin{aligned}
& \dot{x}(t)=\mathbf{A} x(t)+\mathbf{B} u(t)=\left[\begin{array}{cc}
\mathbf{0}^{4 \times 4} & \mathbf{I}^{4 \times 4} \\
-M^{-1}\left(K_{b} B^{T}+B K_{s} B^{T}\right) & -M^{-1}\left(G+C_{b} B^{T}\right)
\end{array}\right] x(t)+\left[\begin{array}{c}
\mathbf{0}^{4 \times 4} \\
M^{-1} B K_{i}
\end{array}\right] u(t) \text { and } \\
& y(t)=\mathbf{C} x(t)=\left[\begin{array}{ll}
B^{T} & \mathbf{0}^{4 \times 4}
\end{array}\right] x(t),
\end{aligned}
$$

where $x(t)=\left[x, y, \theta_{x}, \theta_{y}, \dot{x}, \dot{y}, \dot{\theta}_{x}, \dot{\theta}_{y}\right]^{T}$ is the system state, $y(t)$ is the vector of position sensor measurements in the bearing coordinate system, and $\mathbf{I}^{4 \times 4}$ is an identity matrix. In this case, from Eg. (44), $y(t) \equiv z_{b}(t)$. The control variable $u(t)$ in this formulation is the bearing coil current vector $i(t)$. Section 3.4 will incorporate the power supply behavior to transform the input to voltage control.

\subsection{PD CONTROL}

This section will detail the design of a proportional derivative (PD) controller for the AMB. This is equivalent to the addition of a virtual mechanical spring and virtual damper to the system. This approach has been used in many AMB control systems because of the intuitive nature of the control design. However, it does not take advantage of the performance increases and robustness properties of more advanced control system design techniques that cannot be expressed intuitively as mechanical springs and dampers. ${ }^{3}$

To begin, the PD control law is defined as

$$
\begin{aligned}
i_{x A} & =-k_{P A} x_{A}-k_{D A} \dot{x}_{A}, \\
i_{y A} & =-k_{P A} y_{A}-k_{D A} \dot{y}_{A}, \\
i_{x B} & =-k_{P B} x_{B}-k_{D B} \dot{x}_{B}, \text { and } \\
i_{y B} & =-k_{P B} y_{B}-k_{D B} \dot{y}_{B} .
\end{aligned}
$$

Stated in matrix form,

$$
i(t)=-K_{P} y(t)-K_{D} \dot{y}(t)=-K_{P} B^{T} z(t)-K_{D} B^{T} \dot{z}(t),
$$

where $u(t)=\left[i_{x A}, i_{y A}, i_{x B}, i_{y B}\right]^{T}, K_{P}=\operatorname{diag}\left(k_{P A}, k_{P A}, k_{P B}, k_{P B}\right)$, and $K_{D}=\operatorname{diag}\left(k_{D A}, k_{D A}, k_{D B}, k_{D B}\right)$.

Substituting the PD control law into the state-space equation given by Eg. (49) yields the closed-loop equation

$$
\dot{x}=A x\left[\begin{array}{cc}
\mathbf{0}^{4 \times 4} & \mathbf{I}^{4 \times 4} \\
-M^{-1}\left(K_{b} B^{T}+B K_{s} B^{T}+B K_{i} K_{P} B^{T}\right) & -M^{-1}\left(G+C_{b} B^{T}+B K_{i} K_{D} B^{T}\right)
\end{array}\right] x, \quad x(0)=x_{0} .
$$

The stability and frequency response of the closed-loop system can be analyzed through the closed-loop eigenvalues that solve the equation $\operatorname{det}(\lambda \mathbf{I}-A)=0$, where $\lambda$ are the eigenvalues. Note that the gyroscopic matrix $G$ is a function of the rotor speed $\theta_{z}$, so the eigenvalues will also be a function of the rotor speed. 
Figure 14 show the variations in the closed-loop eigenvalues as a function of rotor speed. The rotor speed varies between 10 and $500 \mathrm{rad} / \mathrm{s}$ (pump operating speed is $377 \mathrm{rad} / \mathrm{s}$ ), the darker points denote higher rotor speeds, and the controller gains are given as

$$
\begin{gathered}
k_{P A}=k_{P B}=-2 \frac{k_{s}}{k_{i}} \text { and } \\
k_{D A}=k_{D B}=\frac{1}{k_{i}} \sqrt{\frac{-m k_{s}}{2}} .
\end{gathered}
$$

As the figure shows, at higher speeds the eigenvalues related to an oscillatory mode move closer to the imaginary axis, and consequently, the oscillatory damping characteristics of the controller degrade at higher rotational speeds.

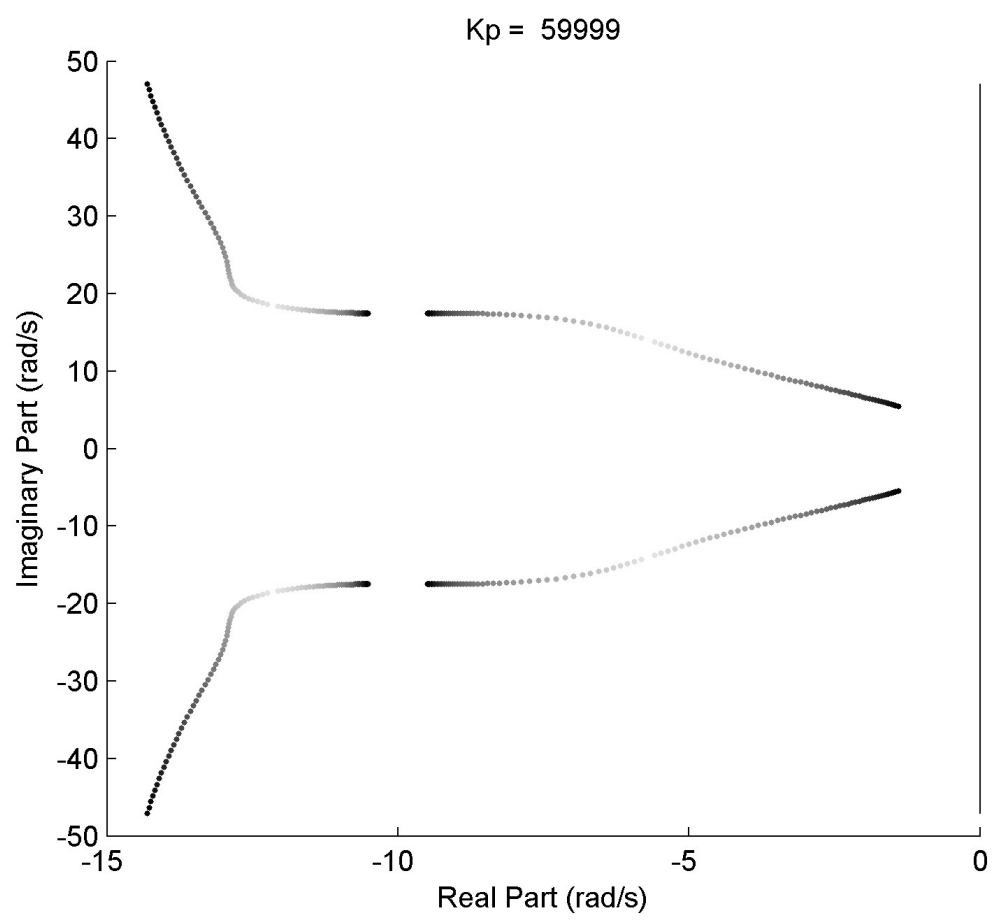

Fig. 14. Closed-loop eigenvalues with a PD controller as a function of rotor speed. Darker points denote a higher rotor speed with a maximum speed of $500 \mathrm{rad} / \mathrm{s}$.

Figures 15 and 16 show the response of the controller to the impact of a 1 in. diameter ball bearing on the impeller.

The system maximum shaft displacement is well within the $1 \mathrm{~mm}$ fluid gap between the rotor and stator. Increasing the proportional and damping parameters can increase the performance of the system at the cost of increasing the sensitivity to sensor and other system noise.

\subsubsection{Current Bias}

It should be noted that in this formulation the bearing coil currents are in $\mathbb{R}$. This is not physically feasible because both a positive and negative coil current will produce a positive force. In reality, the dual 


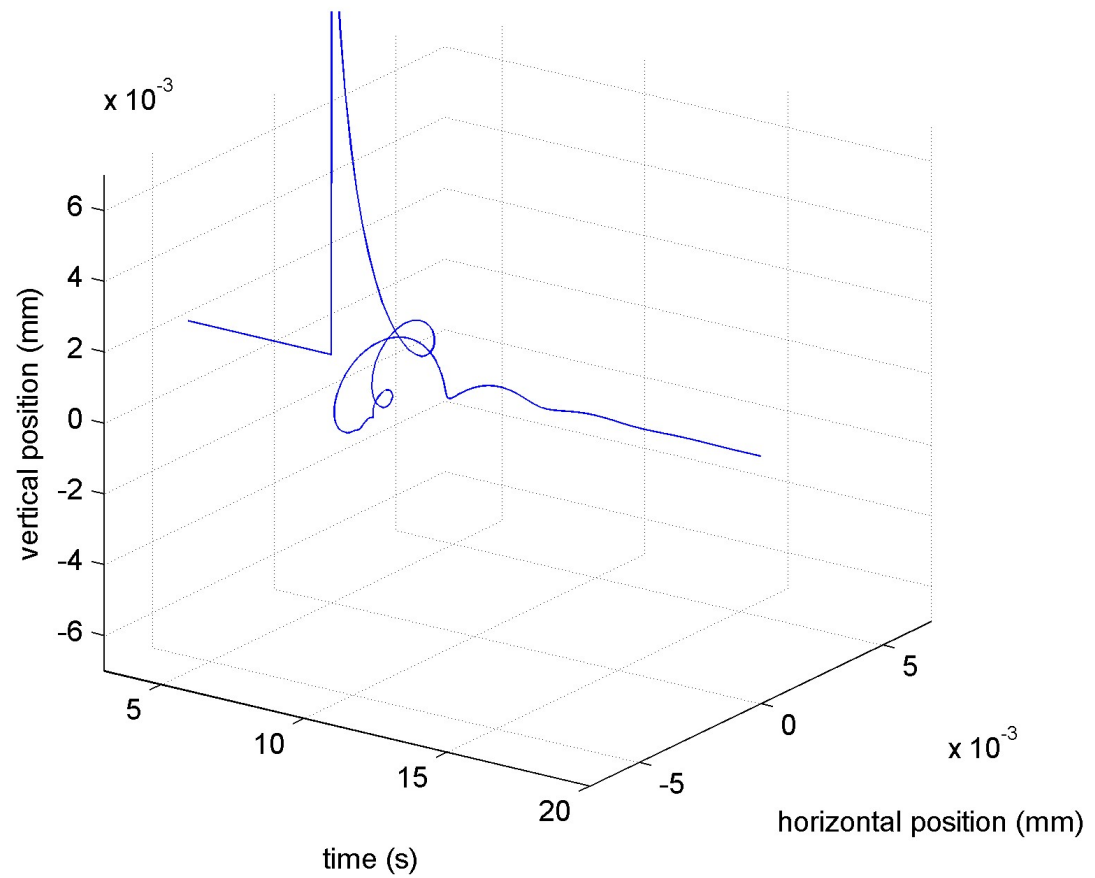

Fig. 15. Rotor COM movement of the closed-loop system to an impact on the impeller.

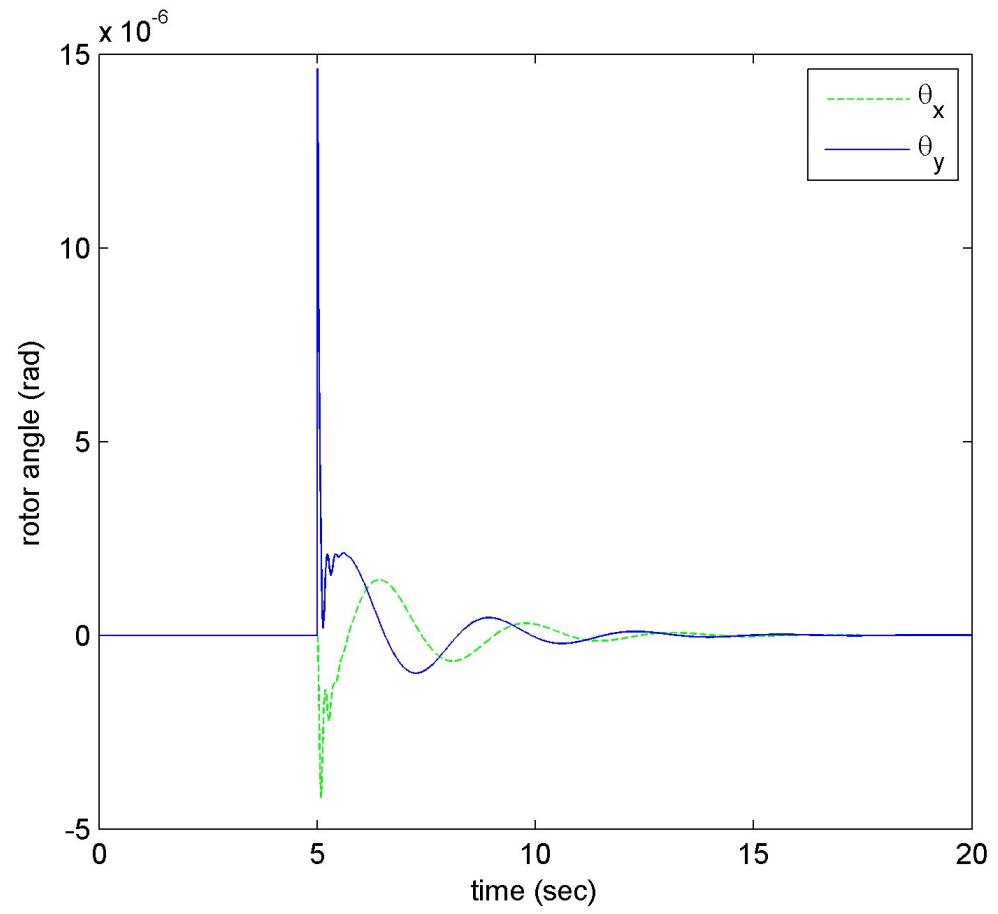

Fig. 16. Rotor COM angular disturbance to an impact on the impeller using PD control 
coils on each bearing axis can only provide a pull force, not a push, hence the need for opposite actuators for each axis. This means that the command current is made up of two individual coil currents as given by

$$
\begin{array}{ll}
i_{x A}=i_{x A}^{+}-i_{x A}^{-}, & i_{x A}^{+} \geq 0, \quad i_{x A}^{-} \geq 0, \\
i_{y A}=i_{y A}^{+}-i_{y A}^{-}, & i_{y A}^{+} \geq 0, \quad i_{y A}^{-} \geq 0, \\
i_{x B}=i_{x B}^{+}-i_{x B}^{-}, \quad i_{x B}^{+} \geq 0, & i_{x B}^{-} \geq 0, \\
i_{y B}=i_{y B}^{+}-i_{y B}^{-}, & i_{y B}^{+} \geq 0, \quad i_{y B}^{-} \geq 0 .
\end{array}
$$

This leads to nonlinearities in the individual coil currents around the zero amplitude currents. This can be overcome by the addition of a bias current to the coils, i.e., $i_{x A}=\left(i_{x A}^{+}+i_{0}\right)-\left(i_{x A}^{-}+i_{0}\right)$. If $i_{0}>\left|i_{x A}\right|$, then the individual coil currents will never reach zero during normal operation. This is analogous to pretensioning springs.

\subsection{PID CONTROL}

The proportional derivative controller can provide stability and rejection of impulse-type disturbances but cannot reject constant disturbances which makes the controller highly sensitive to errors in the physical or estimated parameters for the system. This can be solved by the addition of an integral term into the controller that will remove any steady-state errors in the system. The combined controller is known as a proportional integral derivative (PID) controller.

Applying a local PID controller to each bearing axis is equivalent to

$$
\begin{aligned}
& i_{x A}=-k_{P A} x_{A}-k_{I A} \int_{t} e_{x A}-k_{D A} \dot{x}_{A}, \\
& i_{y A}=-k_{P A} y_{A}-k_{I A} \int_{t} e_{y A}-k_{D A} \dot{y}_{A}, \\
& i_{x B}=-k_{P B} x_{B}-k_{I B} \int_{t} e_{x B}-k_{D B} \dot{x}_{B}, \text { and } \\
& i_{y B}=-k_{P B} y_{B}-k_{I B} \int_{t} e_{y B}-k_{D B} \dot{y}_{B} .
\end{aligned}
$$

where $e_{x A}, e_{y A}, e_{x B}$, and $e_{y B}$ are the errors between the reference inputs and the current system states. In state-space notation, this becomes

$$
u(t)=-\left(K_{P} y+K_{I} \int y+K_{D} \dot{y}\right),
$$

where $K_{P}=\operatorname{diag}\left(k_{P A}, k_{P A}, k_{P B}, k_{P B}\right), K_{I}=\operatorname{diag}\left(k_{I A}, k_{I A}, k_{I B}, k_{I B}\right)$, and $K_{D}=\operatorname{diag}\left(k_{D A}, k_{D A}, k_{D B}, k_{D B}\right)$. This assumes that the sensors are collocated with the AMBs. Substituting Eg. (62) into the closed-loop equation of motion is

$$
M \ddot{z}+\left(G+C_{b} B^{T}\right) \dot{z}+\left(K_{b} B^{T}+B K_{s} C\right) z=-B K_{i} K_{P} B^{T} z-B K_{i} K_{I} \int\left(r(t)-B^{T} z\right)-B K_{i} K_{D} \dot{z} .
$$

Representing Eg. (63) in state-space form requires an augmentation of the system states $x(t)$ by the state $x_{i}(t)=e(t)=r(t)-B^{T} z(t) .{ }^{8}$ The augmented closed-loop differential equation becomes

$$
\begin{aligned}
{\left[\begin{array}{c}
\dot{x} \\
\dot{x}_{i}
\end{array}\right] } & =\left[\begin{array}{cc}
\mathbf{A} & \mathbf{B} K_{I} \\
-B^{T} & \mathbf{0}^{4 \times 8}
\end{array}\right]\left[\begin{array}{c}
x \\
x_{i}
\end{array}\right]+\left[\begin{array}{c}
\mathbf{0}^{8 \times 4} \\
\mathbf{I}^{4 \times 4}
\end{array}\right] r(t) \text { and } \\
y & =\left[\begin{array}{cc}
\mathbf{C} & \mathbf{0}^{4 \times 4}
\end{array}\right]\left[\begin{array}{c}
x \\
x_{i}
\end{array}\right]
\end{aligned}
$$


where $\mathbf{A}, \mathbf{B}$, and $\mathbf{C}$ are from Eg. (49). ${ }^{9}$ The additional states add four additional eigenvalues and modify the existing eigenvalues of $\mathbf{A} .{ }^{10}$

As with the PD control, selection of the controller gains influences the close-loop eigenvalues of the system. The integral term allows the system to track the reference input $r(t)$ and removes any offset error due to disturbances or uncertainties in the linearized model parameters.

\subsection{VOLTAGE CONTROL}

One important aspect of control system design is the choice of the actuation method. In the case of AMBs it is advantageous to use the bearing coil voltage instead of the bearing coil current as the actuation methods. By controlling the voltage, the dynamic characteristics of the power electronics and resistance of the bearing coil to changes in current can be incorporated into the system model. Additionally a voltage-controlled amplifier has a simpler architecture without the need for an underlying current following feedback circuit. However, the change of the input variable from current to voltage also presents greater challenges for the design of the control system and the controller parameters can no longer be interpreted physically as springs and dampers, as is the case with current control. ${ }^{3}$

Changing the input variable from current control to voltage control is equivalent to augmenting the system states with the bearing coil currents $i_{x A}, i_{y A}, i_{x B}, i_{y B}$. The coil currents are governed by the differential equation

$$
v(t)=R i(t)+L \frac{d}{d t} i(t)+K_{u} \dot{x}(t),
$$

where $R=\operatorname{diag}\left(R_{x A}, R_{y A}, R_{x B}, R_{y B}\right)$ is the coil resistance, $L=\operatorname{diag}(L, L, L, L)$ is the coil inductance, and $K_{u}=\operatorname{diag}\left(k_{u}, k_{u}, k_{u}, k_{u}\right)$ is related to the motion-induced voltage. Theoretically it can be shown that

$$
k_{u}=k_{i}
$$

and coil inductance is related to the magnetic bearing coefficients by

$$
L=\frac{k_{i}^{2}}{\left|k_{s}\right|}
$$

due to the translation of energy between electrical and mechanical. ${ }^{11,12}$ This assumes lossless energy conversion but is sufficient for use in designing the controller.

Rearranging Eg. (65) gives

$$
\frac{d}{d t} i(t)=L^{-1} v(t)-L^{-1} R i(t)+L^{-1} K_{i} x(t),
$$

and incorporating it into Eg. (48) yields the coupled differential equations

$$
\begin{aligned}
& M \ddot{z}(t)+\left(G+C_{b} B^{T}\right) \dot{z}(t)+\left(K_{b} B^{T}+B K_{s} B^{T}\right) z(t)=B K_{i} i(t) \text { and } \\
& \frac{d}{d t} i(t)=L^{-1} v(t)-L^{-1} R i(t)-L^{-1} K_{i} \dot{x}(t) .
\end{aligned}
$$

Converting this equation into the state space formulation yields

$$
\begin{aligned}
& \dot{x}(t)=\mathbf{A} x(t)+\mathbf{B} u(t)=\left[\begin{array}{ccc}
\mathbf{0}^{4 \times 4} & \mathbf{I}^{4 \times 4} & \mathbf{0}^{4 \times 4} \\
-M^{-1}\left(K_{b} B^{T}+B K_{s} B^{T}\right) & -M^{-1}\left(G+C_{b} B^{T}\right) & M^{-1} B K_{i} \\
\mathbf{0}^{4 \times 4} & -L^{-1} K_{i} & -L^{-1} R
\end{array}\right]\left[\begin{array}{c}
x(t) \\
\dot{x}(t) \\
i(t)
\end{array}\right]+\left[\begin{array}{c}
\mathbf{0}^{4 \times 4} \\
\mathbf{0}^{4 \times 4} \\
L^{-1}
\end{array}\right] u(t) \\
& y(t)=\mathbf{C} x(t)=\left[\begin{array}{lll}
B^{T} & \mathbf{0}^{4 \times 4} & \mathbf{0}^{4 \times 4}
\end{array}\right] x(t) .
\end{aligned}
$$


This formulation can be used in conjunction with pulse width modulation (PWM) technologies that allow for the implementation of complex control topologies that would otherwise be out of reach.

\subsection{LINEAR QUADRATIC REGULATOR}

PD and PID control treat each bearing axis as independent states. This is directly evident because of the diagonal form of the controller gain matrices. However, it can be seen from the structure of the gyroscopic matrix $G$ and the fluid bearing matrices $K_{b}$ and $C_{b}$ that the motion of each axis is coupled. The two primary motion modes are conical and parallel. ${ }^{3}$ By utilizing the off-diagonal terms of the controller gain matrices, the coupling effect of the gyroscopic and fluid bearing forces can be better controlled.

A linear quadratic regulator (LQR) is an optimal controller that can be used in this instance to obtain the optimal closed-loop performance of the system. Given a linear time-invariant state space equation of the form

$$
\dot{x}(t)=A x(t)+B u(t), \quad x\left(t_{0}\right)=x_{0},
$$

the designer seeks to minimize the quadratic cost function

$$
J=\frac{1}{2} \int_{t 0}^{t^{f}} x^{T} Q x+u^{T} R u \mathrm{~d} t
$$

where $Q=Q^{T}$ and $R=R^{T}$ are weighting matrices specified by the designer. This cost function is analogous to minimizing the overall energy of the system. The state weighting matrix $Q$ is used to minimize deviations due to disturbances, and the input weighting matrix $R$ is used to reduce the control effort. There are many commercially available tools for solving this minimization problem given proper definition of the system dynamics.

\subsubsection{Observer}

One key aspect of the LQR controller is that it requires full-state feedback. Due to the high-temperature and extreme environment, it is unlikely that direct measurements of the position and velocity of the rotor will be available. In this instance, a state estimator can be employed to estimate the unmeasured states based on knowledge of the system dynamics and the available sensor measurements. The estimator (also known as an Luenberger observer) equations are

$$
\begin{aligned}
& \dot{\hat{x}}(t)=A \hat{x}(t)+B u(t)+L[y(t)-\hat{y}(t)] \text { and } \\
& \hat{y}(t)=C \hat{x}(t),
\end{aligned}
$$

where $\hat{x}(t)$ is the estimated state, $\hat{y}(t)$ is the expected output from the estimated state, $L$ is the observer gain, and $e(t)=y(t)-\hat{y}(t)$ is the error between the sensor signals and the estimated sensor signals. The objective is to define the observer gain $L$ such that the observer error $e(t) \rightarrow 0$ as $t \rightarrow \infty$. This can be easily done by pole placement techniques so that the eigenvalues of the observer are to the left of the eigenvalues of the closed-loop system, or in other words, the observer bandwidth must exceed the closed-loop system bandwidth. This ensures that the observer which is implemented virtually can respond quickly enough to the system. 



\section{SIMULATION RESULTS}

In this section, simulation results for various controller architectures and design theories are presented to compare controller performance. The system is subjected to an input disturbance that is equivalent to a $106 \mathrm{~N}(23.83 \mathrm{lbf})$ static load. This load is applied instantaneously at $t=1 \mathrm{~s}$, and the response of the closed-loop system to the disturbance is calculated. In the simple case of the PD controller, the disturbance force is simulated as both the static disturbance and an impulse response lasting 0.01 seconds. This is to contrast the PD and PID steady state error. The simulations were built in MATLAB $^{\complement}$ and Simulink ${ }^{\complement}$ using the equations presented in Sect. 2 and Sect. 3. All numbers used in the simulations were taken directly from the conceptual rotor design and are listed in Tables 1, rotor.parameters, and mag.param.

\subsection{PD CONTROL}

For the PD controller, the proportional gain was chosen to be $k_{P}=2.5 \times 10^{7}$ and the derivative gain was chosen as $k_{D}=6 \times 10^{4}$. This proportional gain corresponds to a frequency of $985 \mathrm{rad} / \mathrm{s}$, which is well above the operating angular speed of the rotor. Figure 17 shows the variation in the system's closed-loop eigenvalues as the rotor speed is varied between $10 \mathrm{rad} / \mathrm{s}$ and $500 \mathrm{rad} / \mathrm{s}$. As Fig. 17 shows, the controller has decreased the sensitivity of the system response to changes in rotational speed.

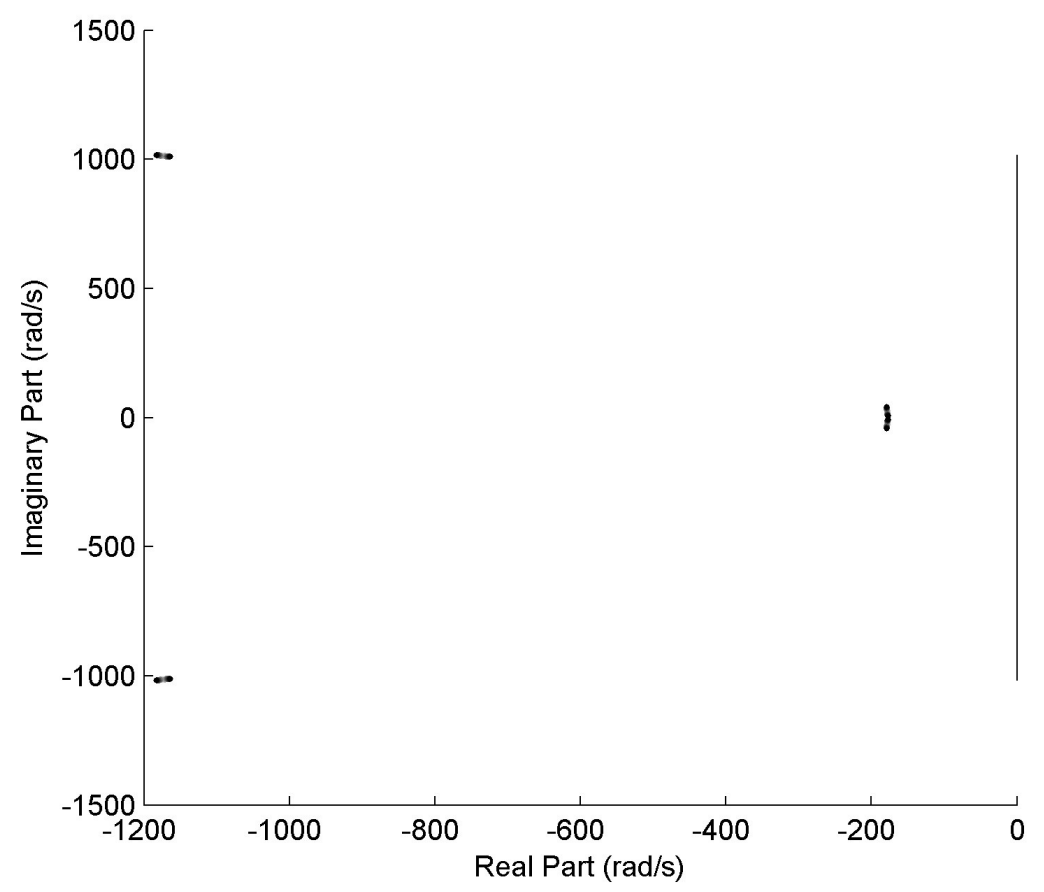

Fig. 17. Variations in the closed-loop eigenvalues for the PD controller as a function of rotor speed. The lighter points denote lower speeds, and conversely, the darker points denote higher rotor speeds. The rotor speed range is $10 \mathrm{rad} / \mathrm{s}$ to $500 \mathrm{rad} / \mathrm{s}$.

Figure 18 show the movement of the rotor COM to an impulse disturbance on the impeller that lasts for $0.01 \mathrm{~s}$ and has a force amplitude of $106 \mathrm{~N}$. The rotor remains well withing the physical constraints imposed 
by the fluid gap and returns to nominal operation within a fraction of a second without excessive overshoot or oscillations.

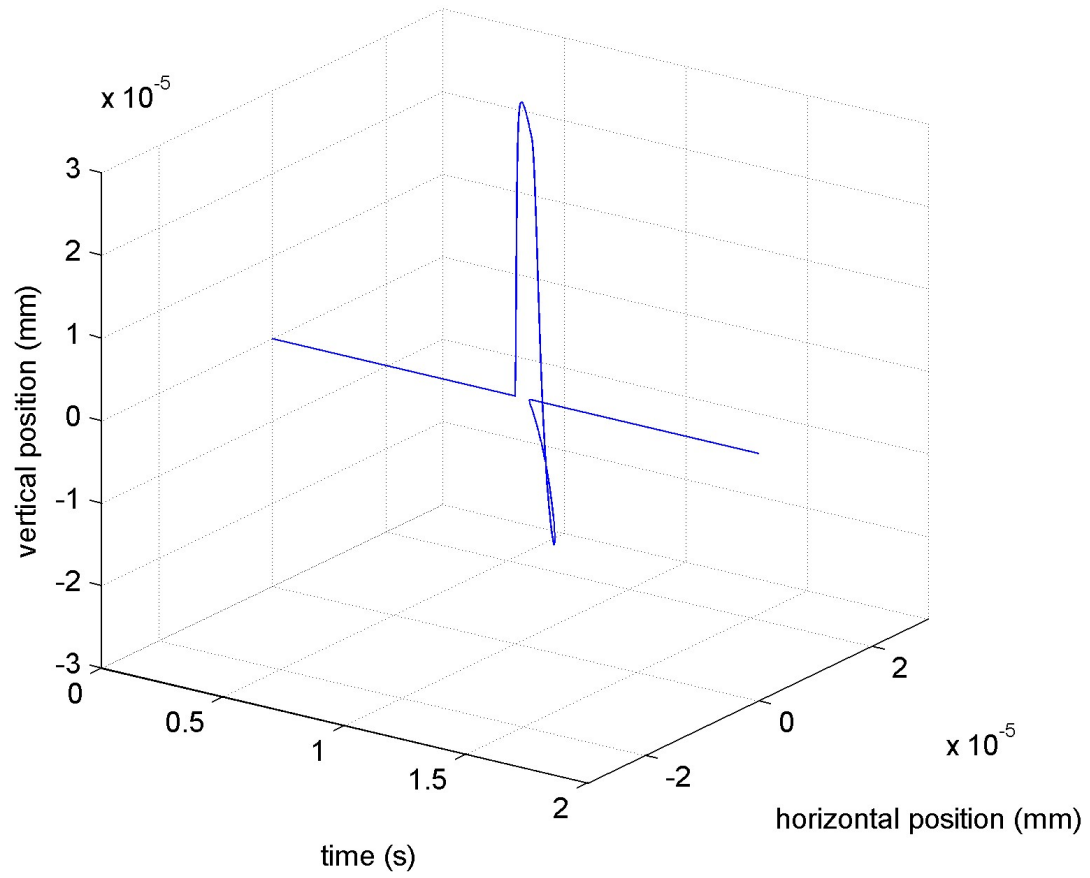

Fig. 18. Rotor COM translational disturbance to an impulse impact on the rotor with a PD controller.

The lack of an integral term in the controller means that the system will alway exhibit a steady-state error in response to unmodeled dynamics or external forces. Figure 19 show the movement of the rotor $\mathrm{COM}$ in response to a constant disturbance with a magnitude of $106 \mathrm{~N}$. After the transients settle, the rotor is offset and the shaft maintains a small angle with respect to the geometric center, as shown in Fig. 20.

Figures 21 and 22 depict the coil current for the radial magnetic bearings. As they show, the coil currents do not rise above 2 A. 


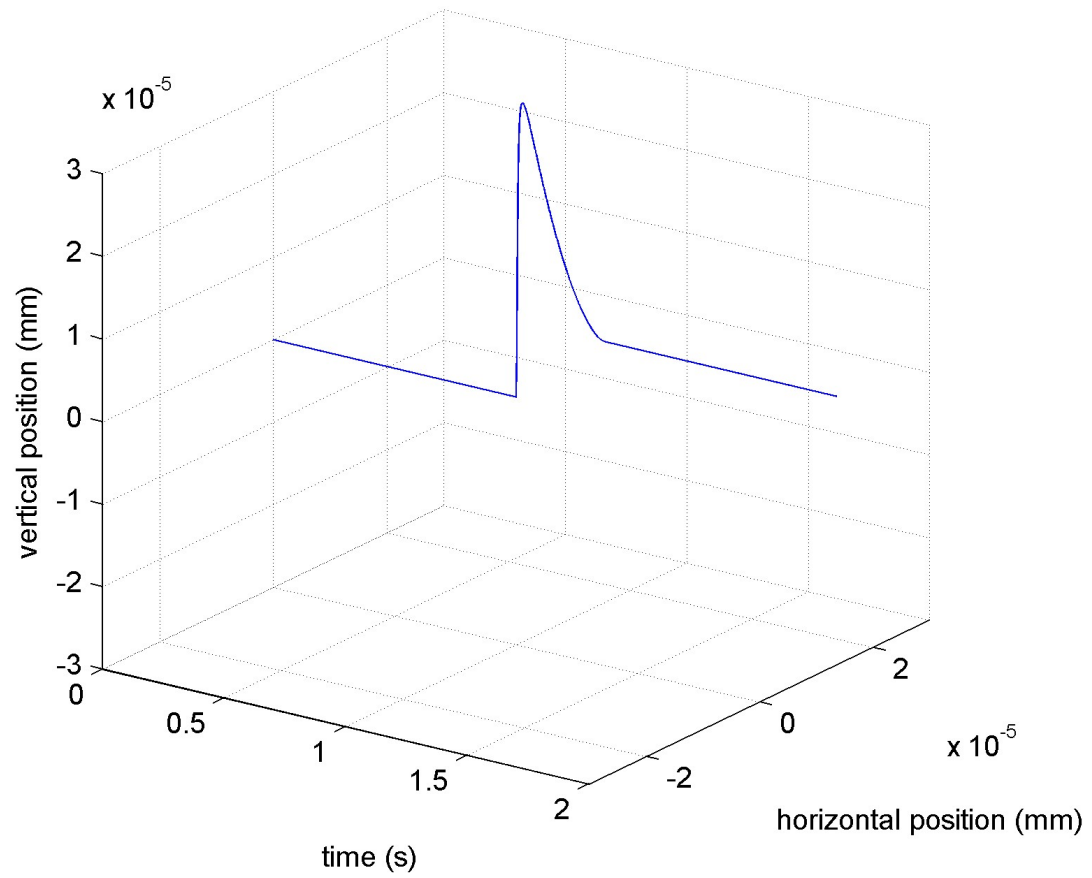

Fig. 19. Rotor COM translational movement to a constant disturbance on the rotor with a PD controller.

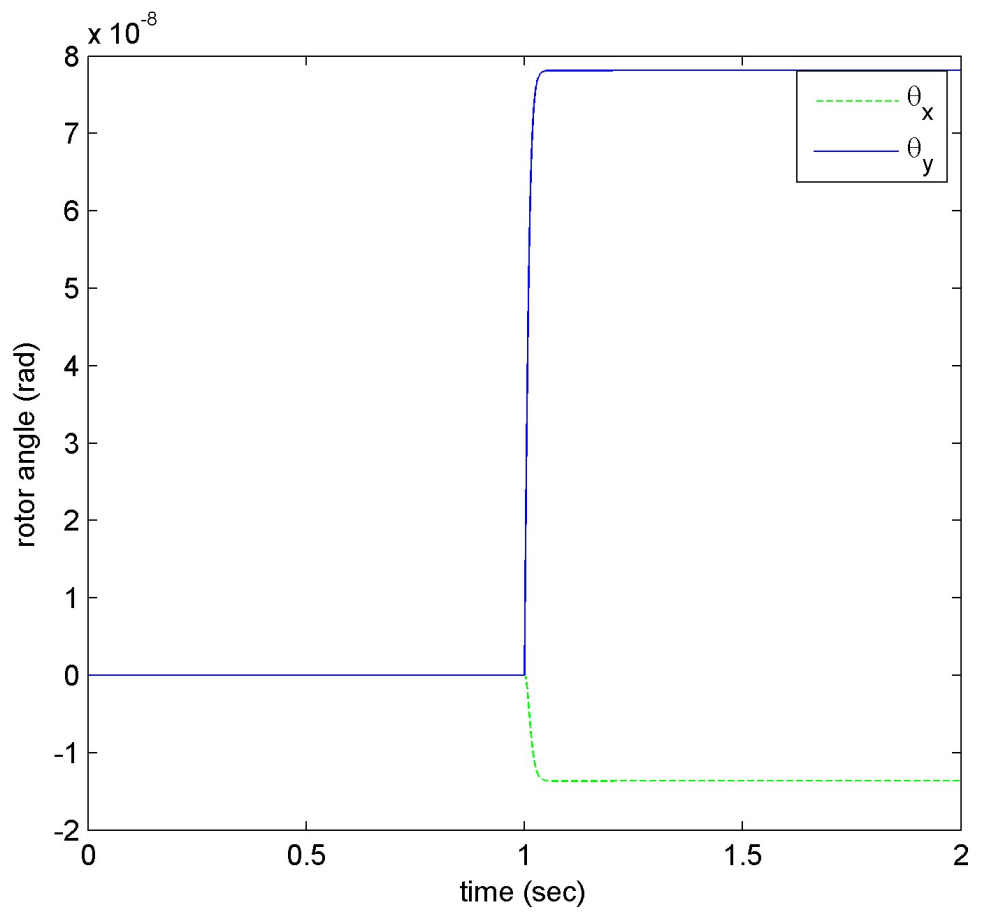

Fig. 20. Rotor rotational movement about the COM in response to a constant disturbance on the rotor with a PD controller. 


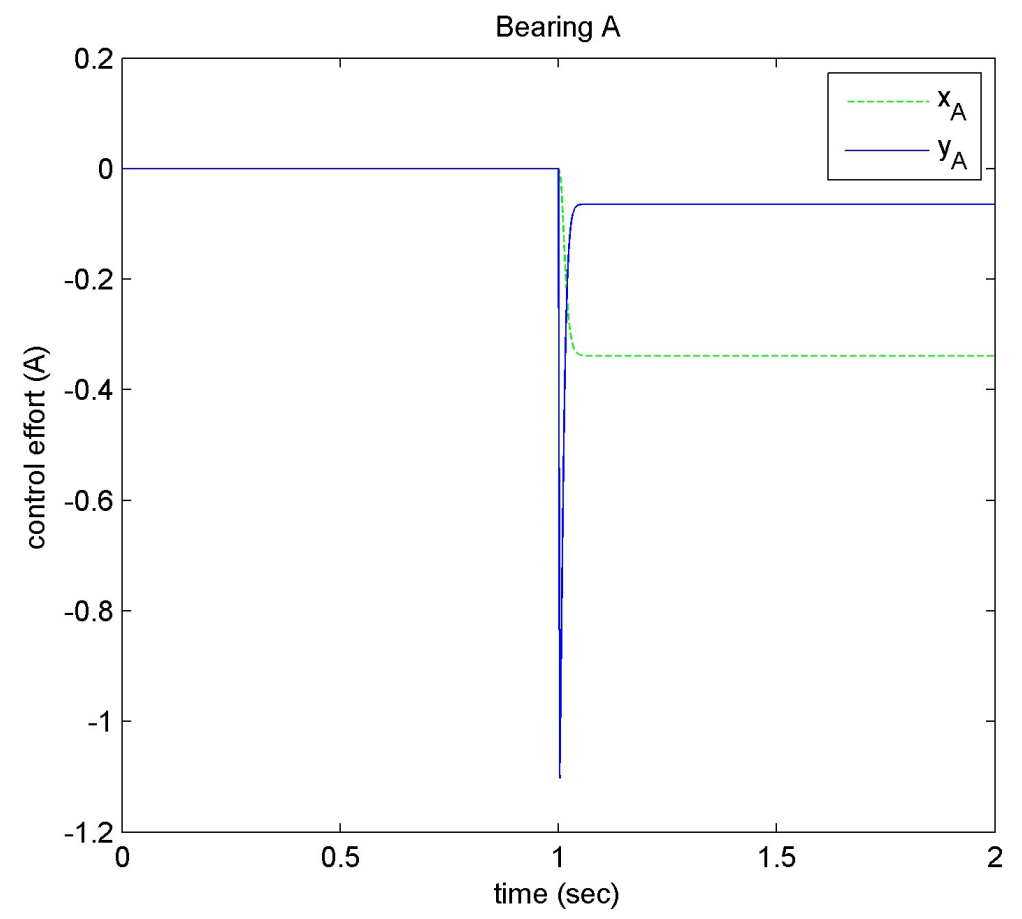

Fig. 21. Coil input currents in amps for the AMB denoted by $A$ using a PD controller. Physically, the $A$ bearing is on the opposite end of the shaft from the impeller.

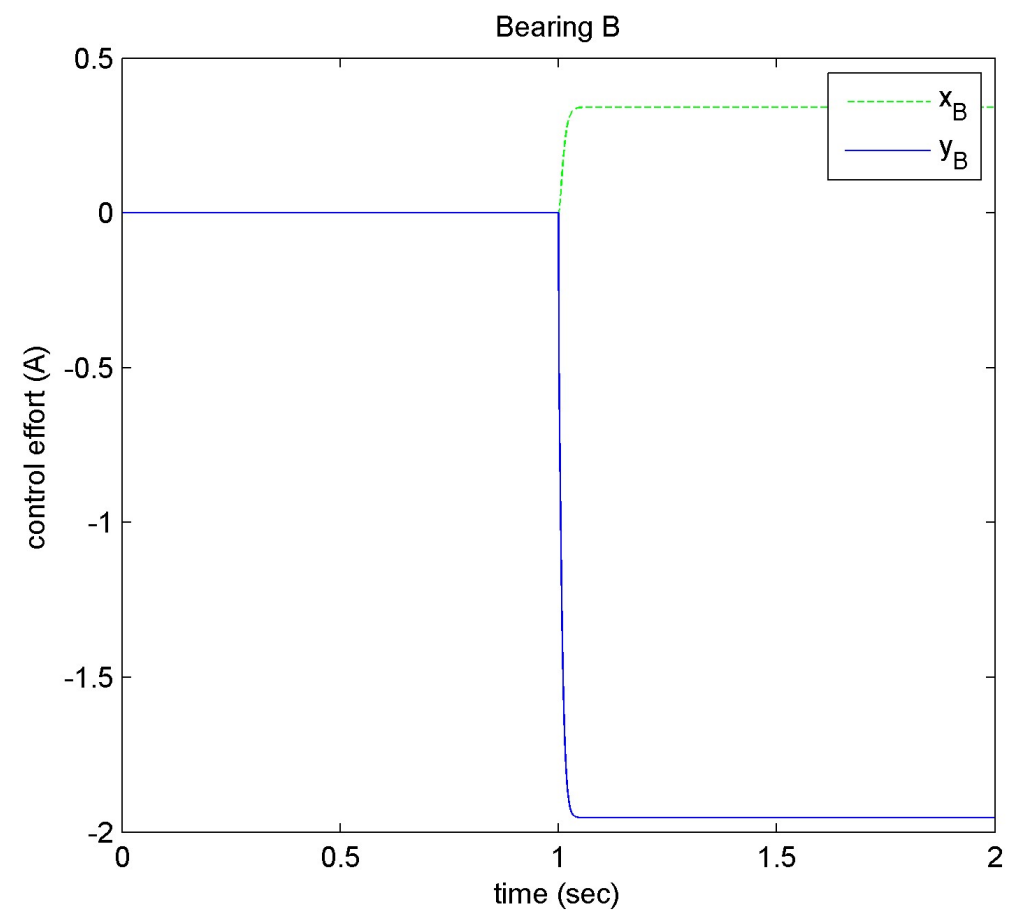

Fig. 22. Coil input currents in amps for the AMB denoted by $B$ using a PD controller. Physically, the $B$ bearing is on the end of the shaft nearest to the impeller. 


\subsection{PID CONTROL}

The addition of an integral component to the controller removes any steady state error due to disturbances or other system unknown. A comparison of Fig. 17 and Fig. 23 shows that the integral controller does not change the eigenvalues of the original system, but adds eigenvalues close to the origin that correspond to the integrator dynamics. The proportional gain and derivative gain are identical to those chosen for the PD controller, and the integral gain was chosen as $k_{I}=1 \times 10^{7}$.

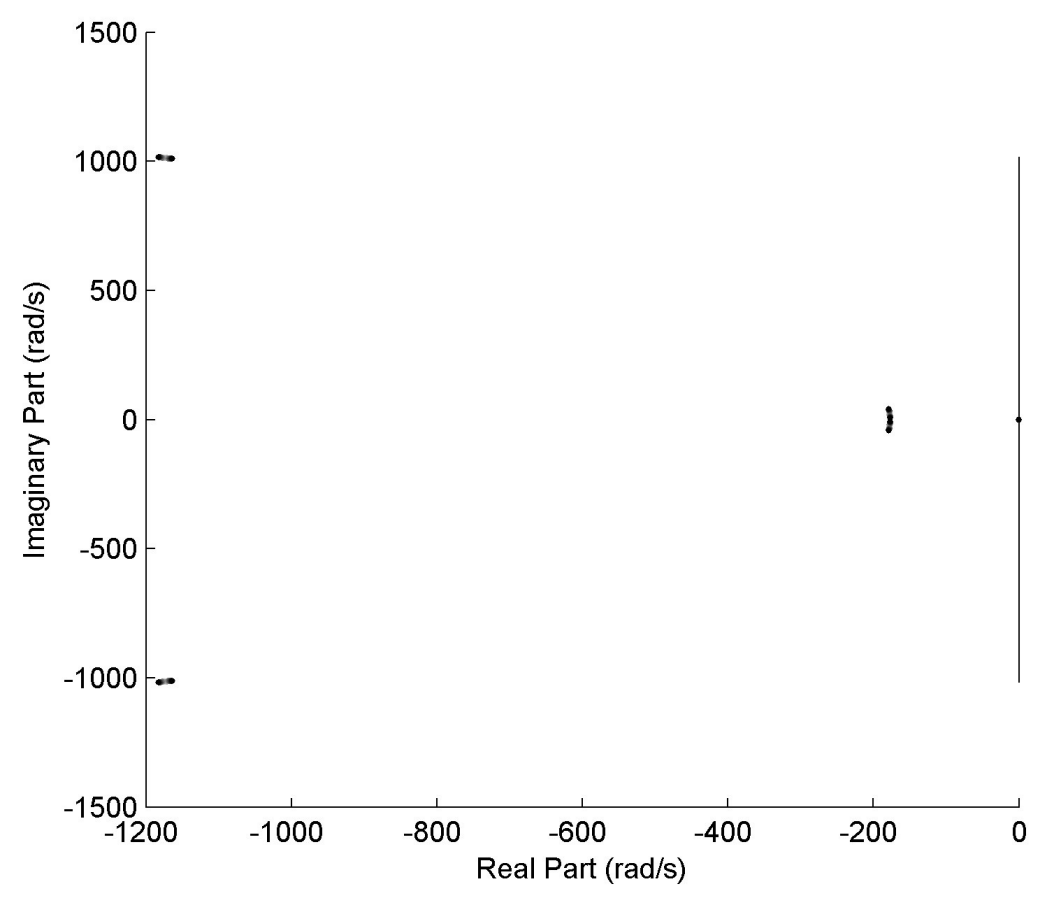

Fig. 23. Variations in the closed-loop eigenvalues for the PID controller as a function of rotor speed. The lighter points denote lower speeds, and conversely, the darker points denote higher rotor speeds. The rotor speed range is $10 \mathrm{rad} / \mathrm{s}$ to $500 \mathrm{rad} / \mathrm{s}$.

Figure 24 shows the movement of the rotor COM to a constant $106 \mathrm{~N}$ disturbance that is applied instantaneously at 1 second. Comparing Fig. 19 and Fig. 24 illustrates the steady-state disturbance rejection properties of the integral portion of the controller. Figure 25 depicts the angular movement of the rotor about the COM.

Figures 26 and 27 show that while there is an increase in the maximum coil current due to the integral term, the difference is small. As expected, there is a non-zero steady-state coil current to offset the disturbance force; however, unlike the PD controller, the off-axis currents approach zero as the system transients die out. 


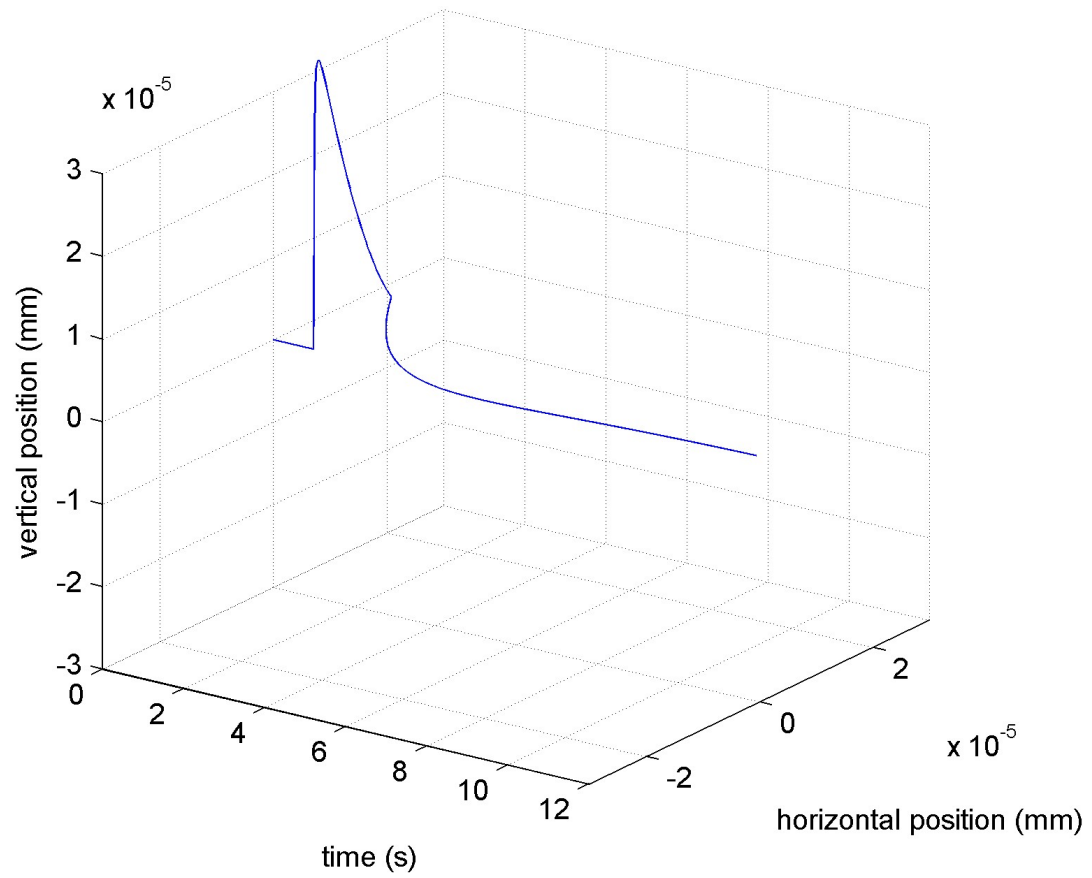

Fig. 24. Rotor COM translational movement to a constant disturbance on the rotor with a PID controller.

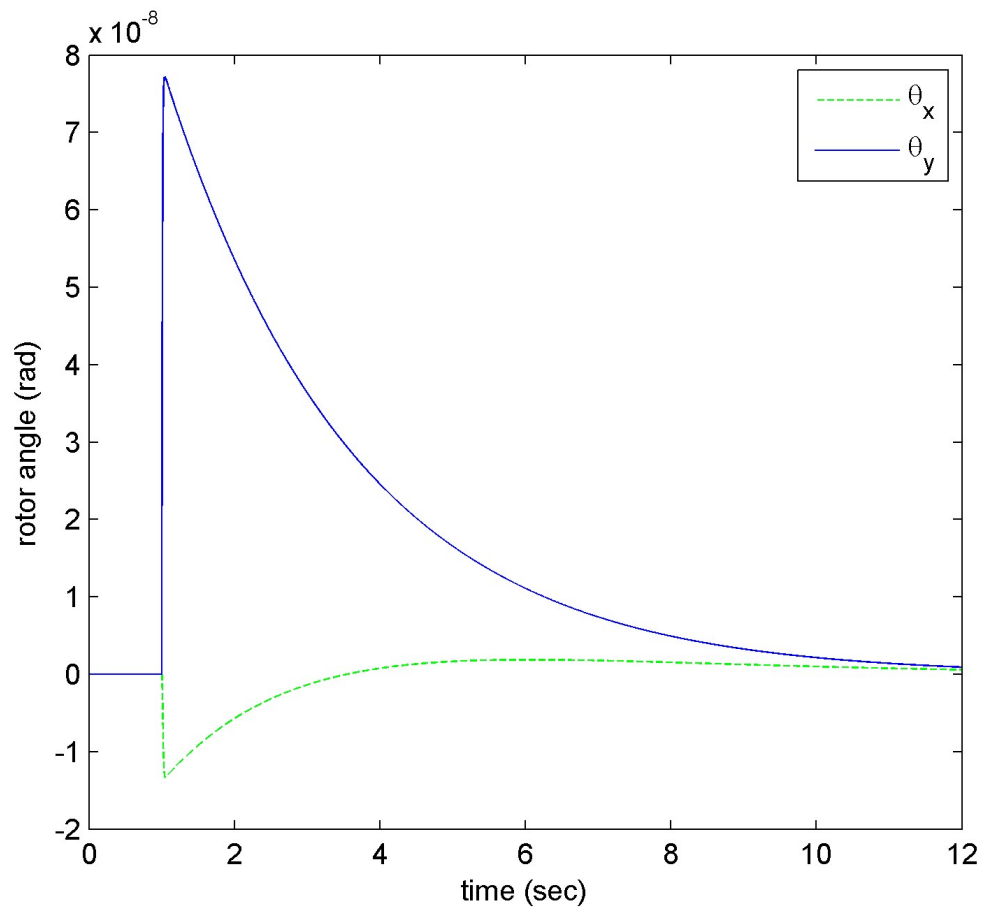

Fig. 25. Rotor rotational movement about the COM in response to a constant disturbance on the rotor with a PID controller. 


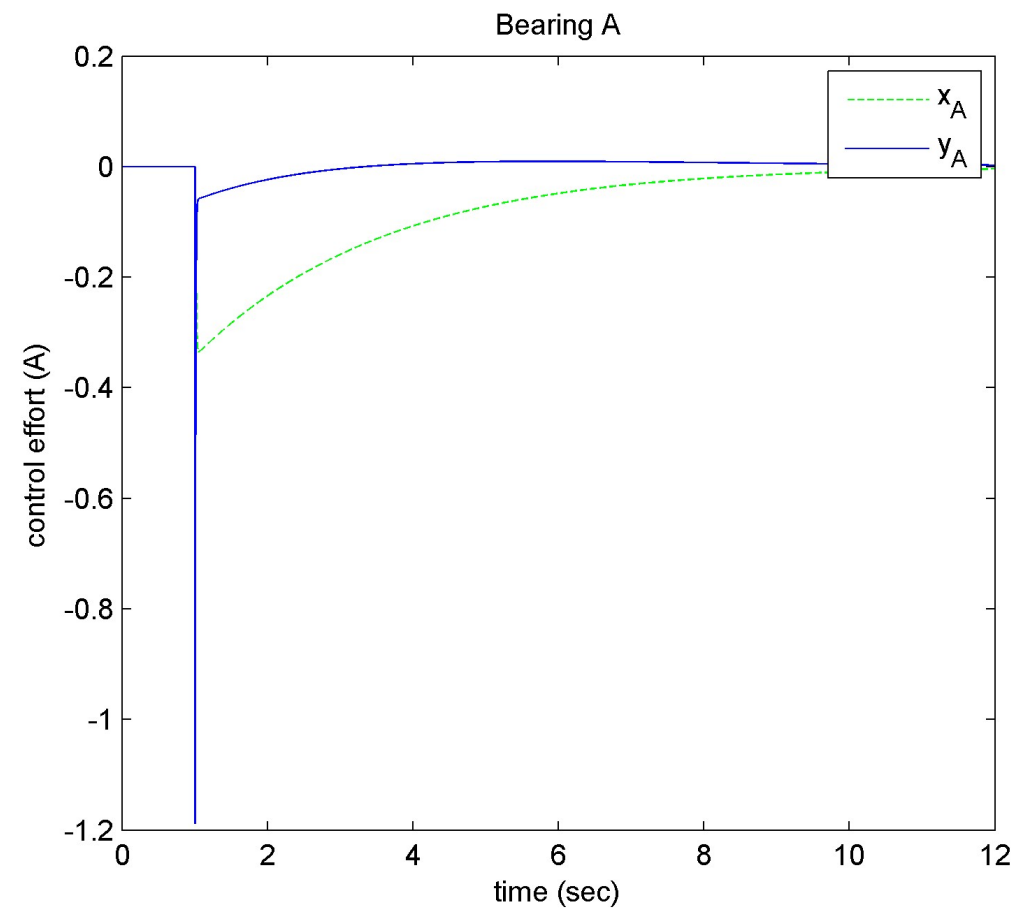

Fig. 26. Coil input currents in amps for the AMB denoted by $A$ using a PID controller. Physically, the $A$ bearing is on the opposite end of the shaft from the impeller.

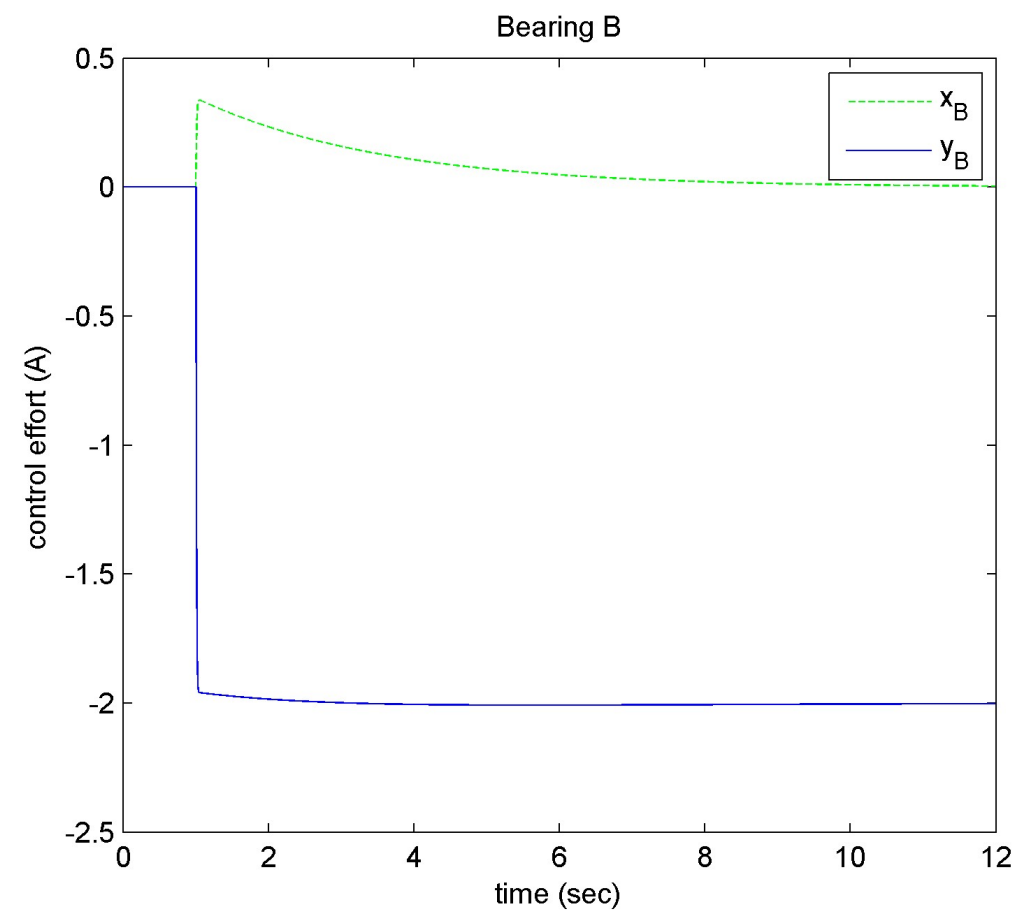

Fig. 27. Coil input currents in amps for the AMB denoted by $B$ using a PID controller. Physically, the $B$ bearing is on the end of the shaft nearest to the impeller. 


\subsection{LQR CONTROL}

To develop the LQR control, the weighting matrices $R$ and $Q$ given by Eg. (72) need to be specified. The coefficients of these matrices determine the balance between the control effort and the transient error of the optimized controller. The matrix to minimize the energy of the system states is given by

$$
Q=\left[\begin{array}{cccccccc}
1 \times 10^{18} & 0 & 0 & 0 & 0 & 0 & 0 & 0 \\
0 & 1 \times 10^{18} & 0 & 0 & 0 & 0 & 0 & 0 \\
0 & 0 & 1 \times 10^{14} & 0 & 0 & 0 & 0 & 0 \\
0 & 0 & 0 & 1 \times 10^{14} & 0 & 0 & 0 & 0 \\
0 & 0 & 0 & 0 & 1 \times 10^{6} & 0 & 0 & 0 \\
0 & 0 & 0 & 0 & 0 & 1 \times 10^{6} & 0 & 0 \\
0 & 0 & 0 & 0 & 0 & 0 & 1 \times 10^{6} & 0 \\
0 & 0 & 0 & 0 & 0 & 0 & 0 & 1 \times 10^{6}
\end{array}\right],
$$

and the matrix to minimize the energy of the control inputs is given by

$$
R=\mathbf{I}^{4 \times 4} \text {. }
$$

Figure 28 shows the closed-loop eigenvalues of the optimal LQR controller.

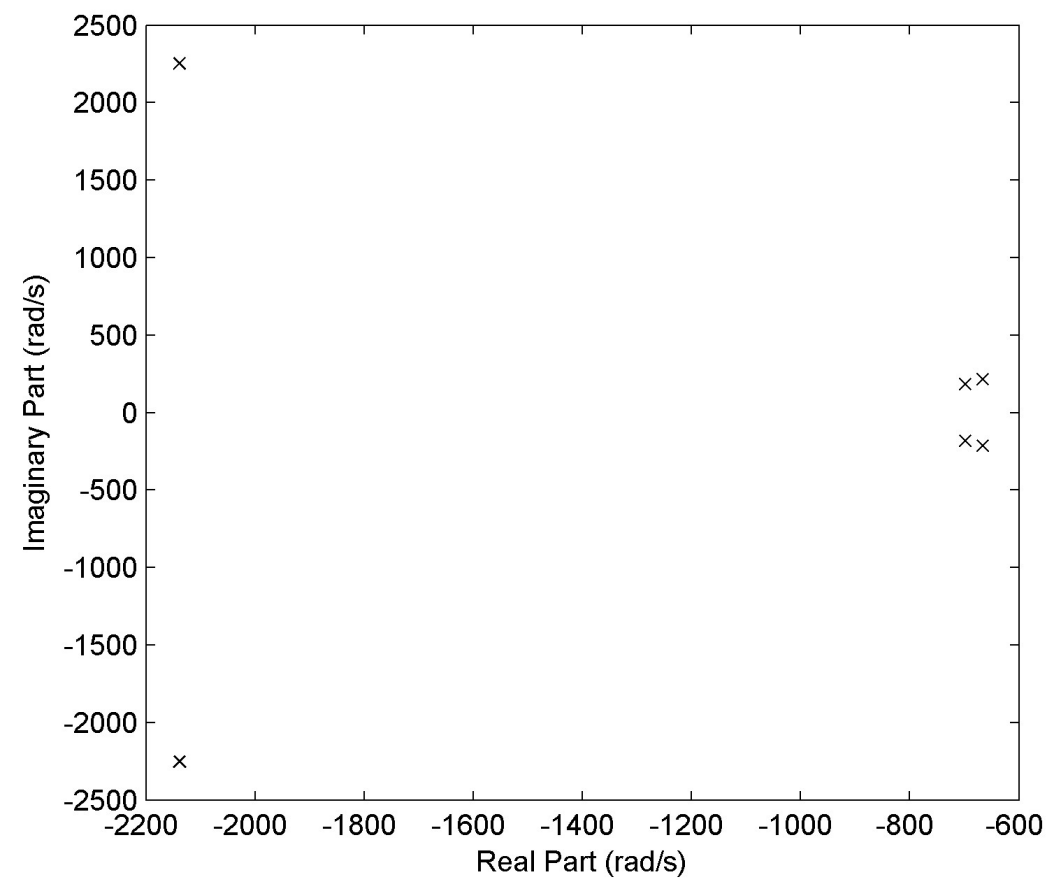

Fig. 28. The closed-loop eigenvalues for the LQR controller.

As Fig. 29 shows, the performance of the LQR controller is superior to that of the PD controller because it controls the coupled behavior of the system, unlike the PD controller, which assumes that each state is independent. Figure 30 shows the rotation of the shaft around the COM.

Figures 31 and 32 show that the LQR control effort maximum magnitude is smaller than the PD or PID controller, while the maximum deviation of the rotor is an order of magnitude smaller for the LQR controller, as shown in Fig. 29. 


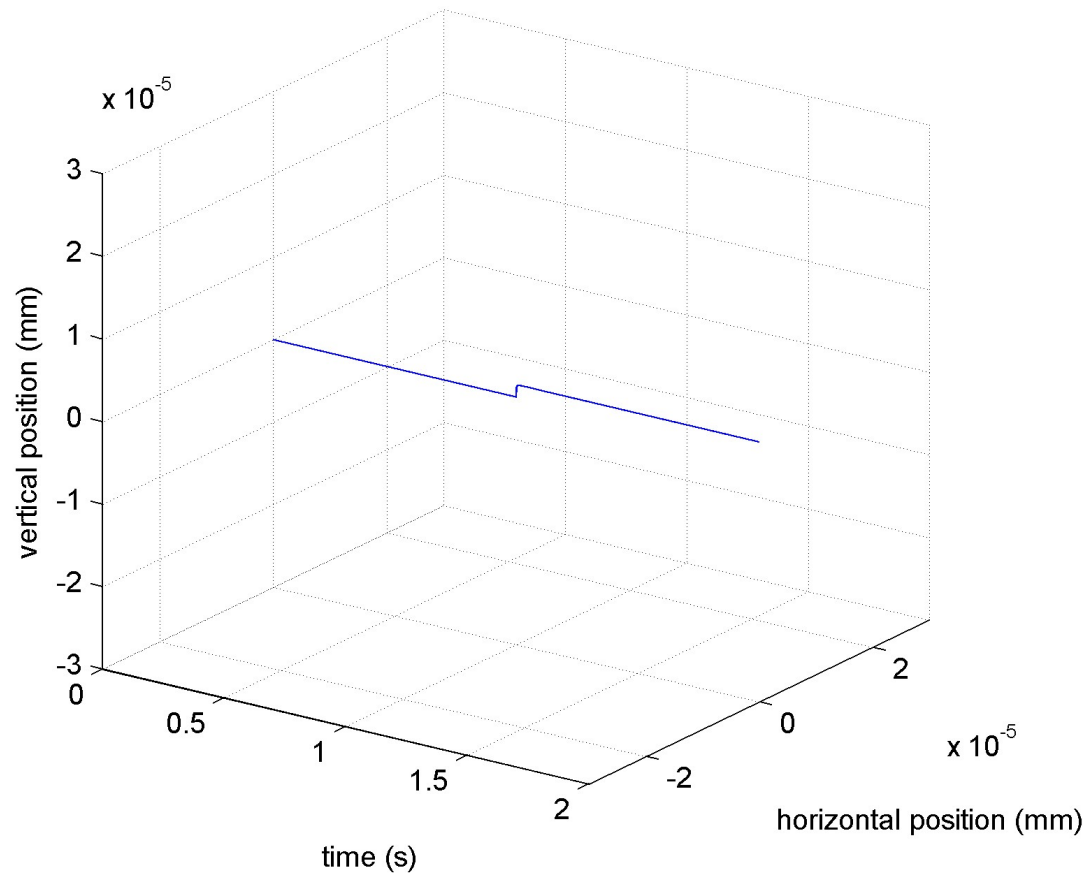

Fig. 29. Rotor COM translational movement to a constant disturbance on the rotor with an LQR controller.

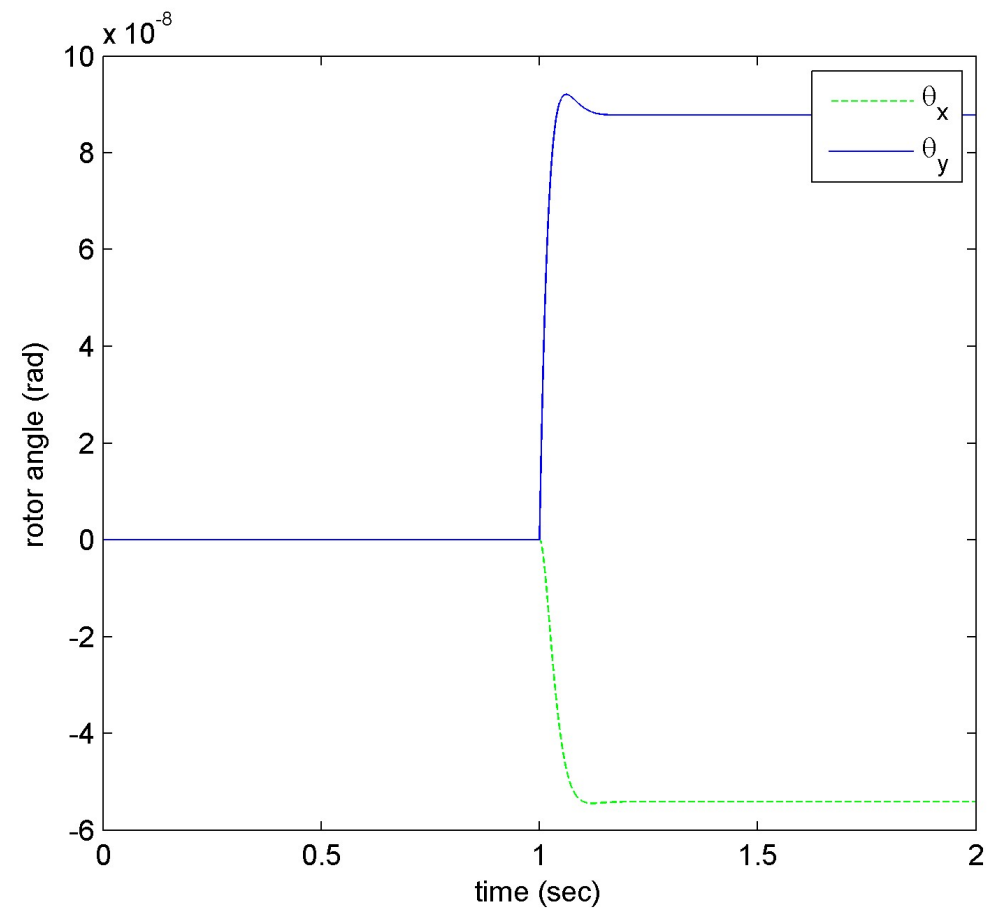

Fig. 30. Rotor rotational movement about the COM in response to a constant disturbance on the rotor with an LQR controller. 


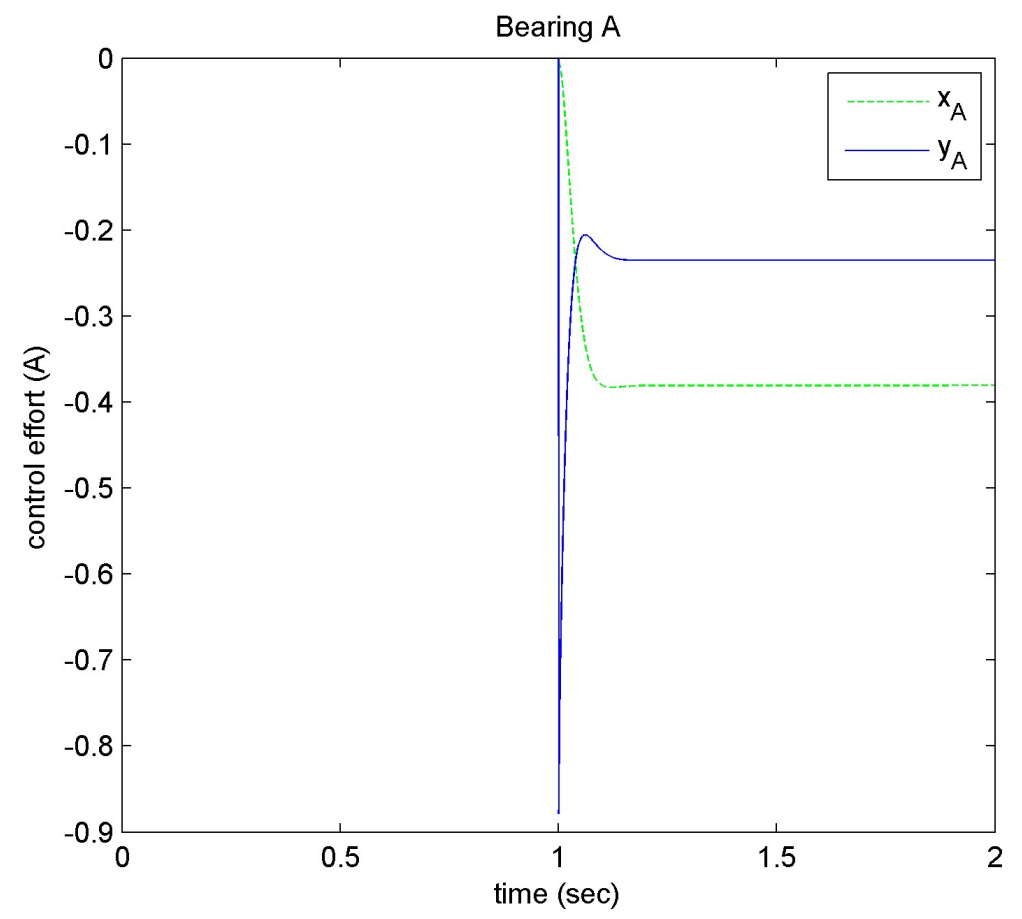

Fig. 31. Coil input currents in amps for the AMB denoted by $A$ using an LQR controller. Physically, the $A$ bearing is on the opposite end of the shaft from the impeller.

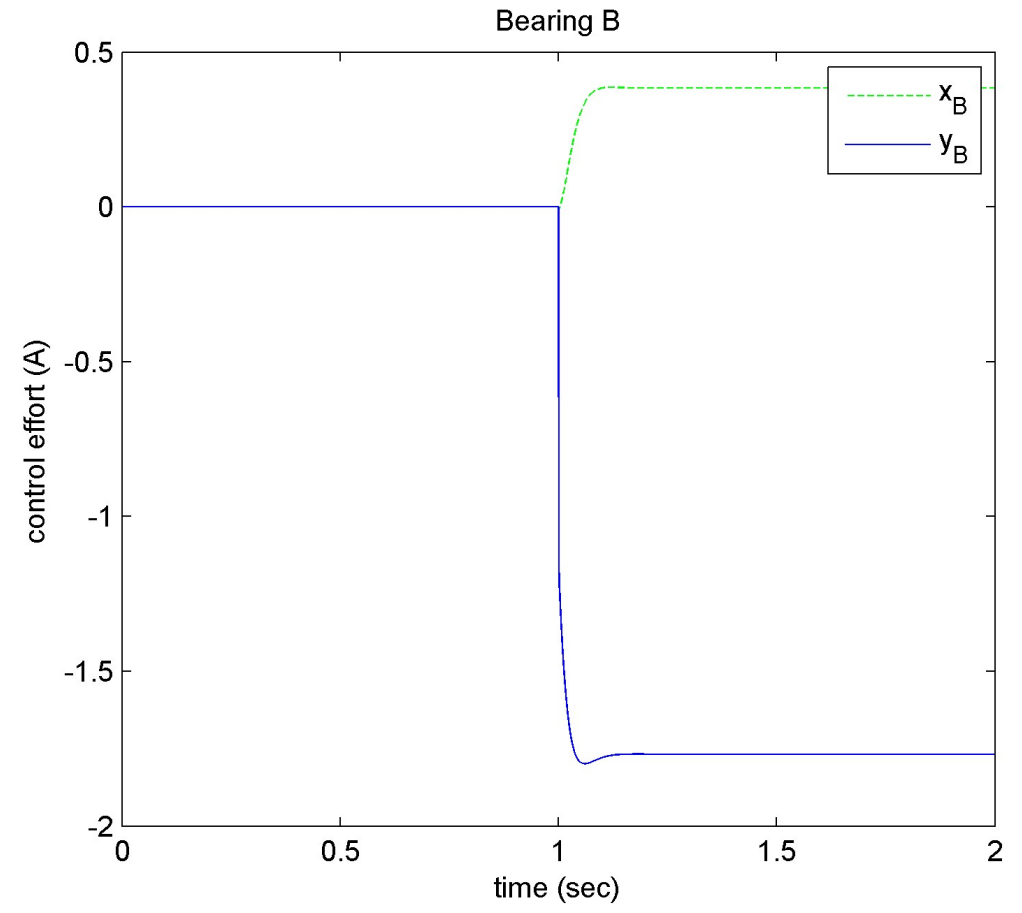

Fig. 32. Coil input currents in amps for the AMB denoted by $B$ using an LQR controller. Physically, the $B$ bearing is on the end of the shaft nearest to the impeller. 


\subsection{LQR CONTROL WITH INTEGRATOR}

Similarly to the PD controller, the LQR controller does not contain an integrator, which means that the LQR controller will exhibit steady-state errors in response to disturbances, parameter uncertainties, and unmodeled dynamics. The addition of an integrator into the LQR controller with appropriate gains will remove the steady-state error and allow the controller to track a reference signal. Figure 33 shows the additional eigenvalues related to the integrator and the slight movement of the eigenvalues related to the system dynamics due to the cost balancing mechanism of the optimization.

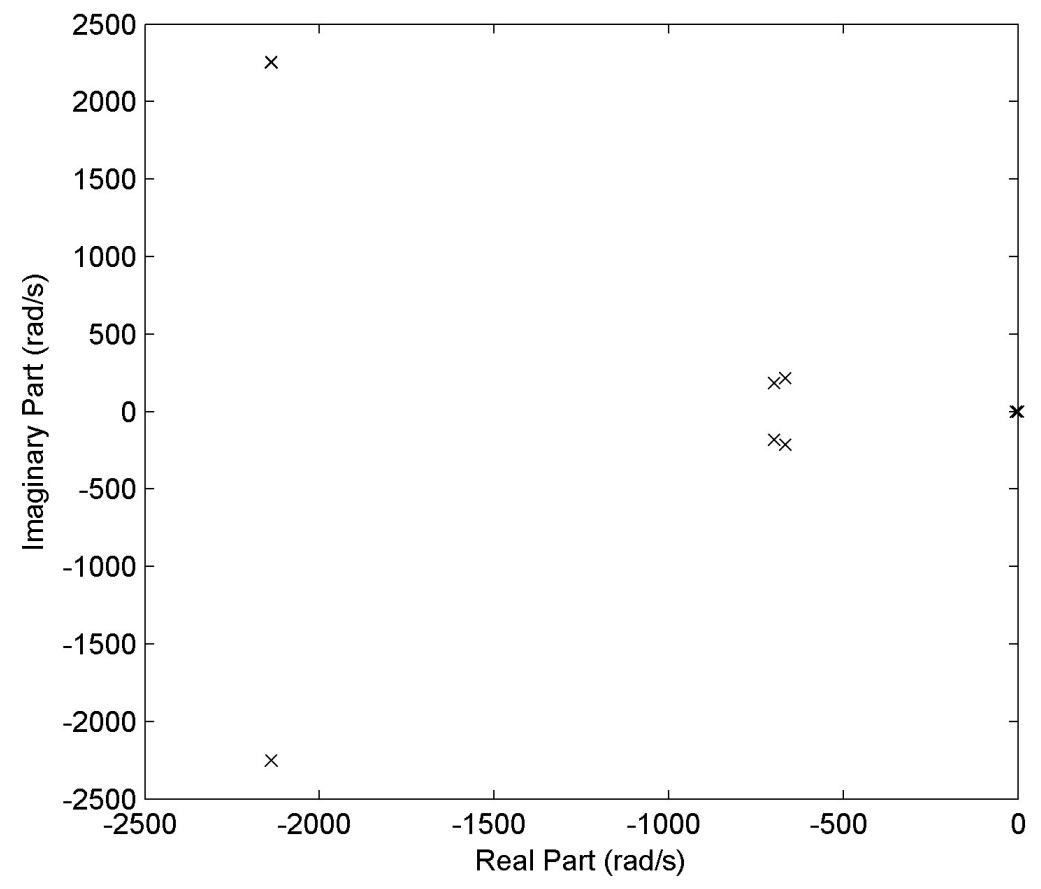

Fig. 33. The closed-loop eigenvalues for the LQR controller with an integrator.

As Fig. 34 shows, the LQR controller with the integrator exhibits a significant improvement over the PID controller in both maximum deviation of the rotor and settling time in response to a constant disturbance.

Figure 35 shows that the maximum angular deviation of the shaft in response to the disturbance is similar for both the PID and LQR with integrator controllers. However, the settling time of the LQR controller with the integrator is an order of magnitude better.

Figures 36 and 37 show that the addition of the integrator into the controller increases the control effort slightly but that the maximum amplitude of the control effort is almost identical to that required by the PID controller. 


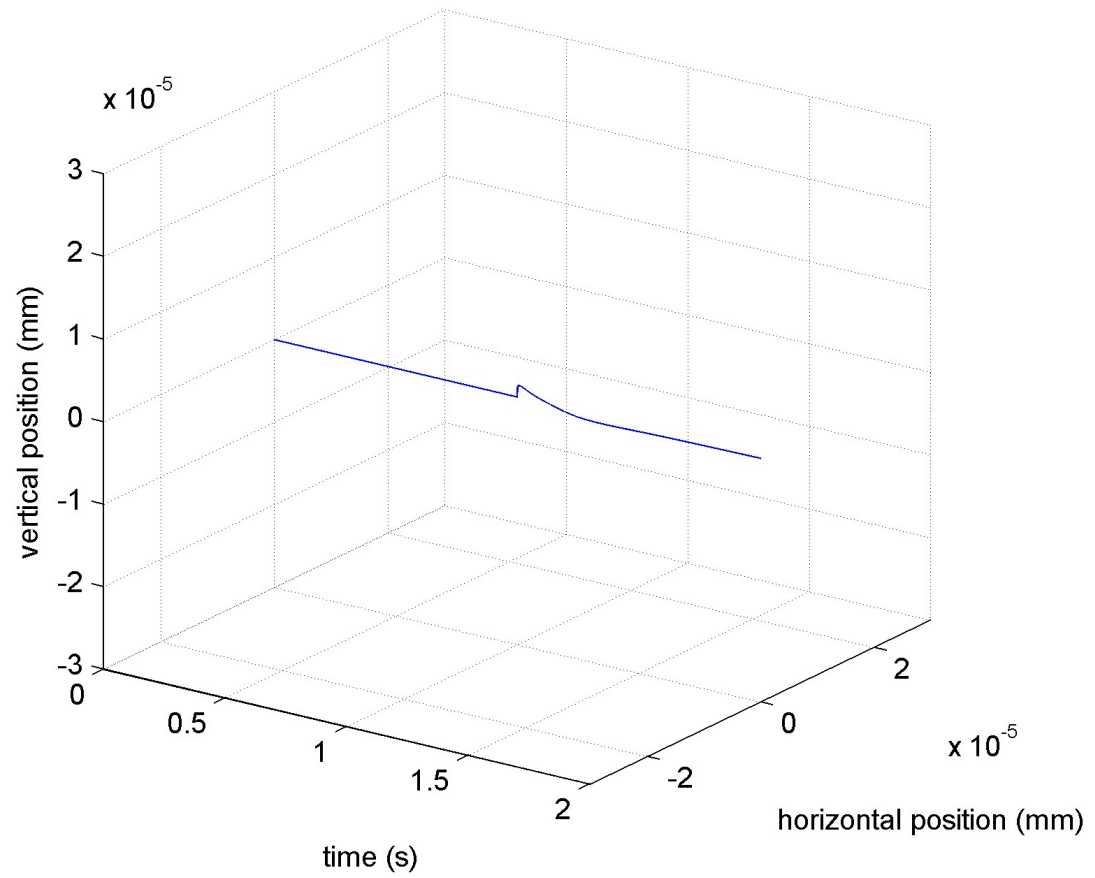

Fig. 34. Rotor COM translational movement to a constant disturbance on the rotor with an LQR controller with an integrator.

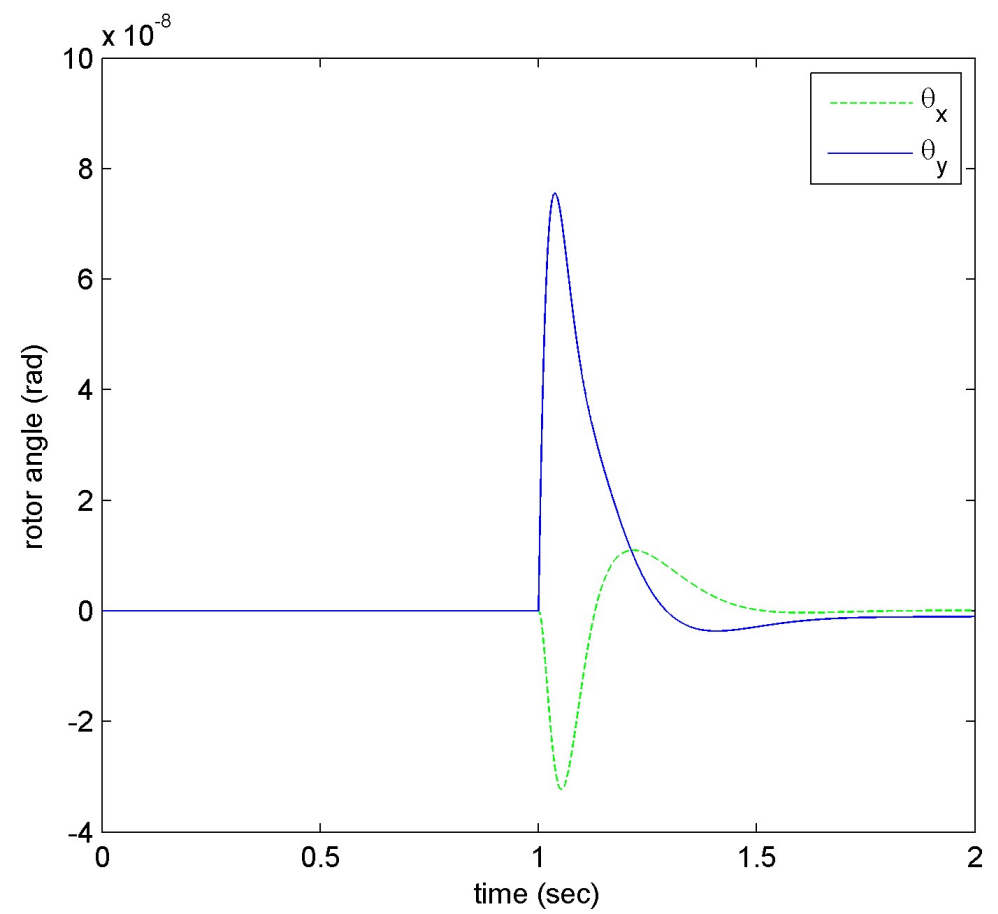

Fig. 35. Rotor rotational movement about the COM in response to a constant disturbance on the rotor with an LQR controller with an integrator. 


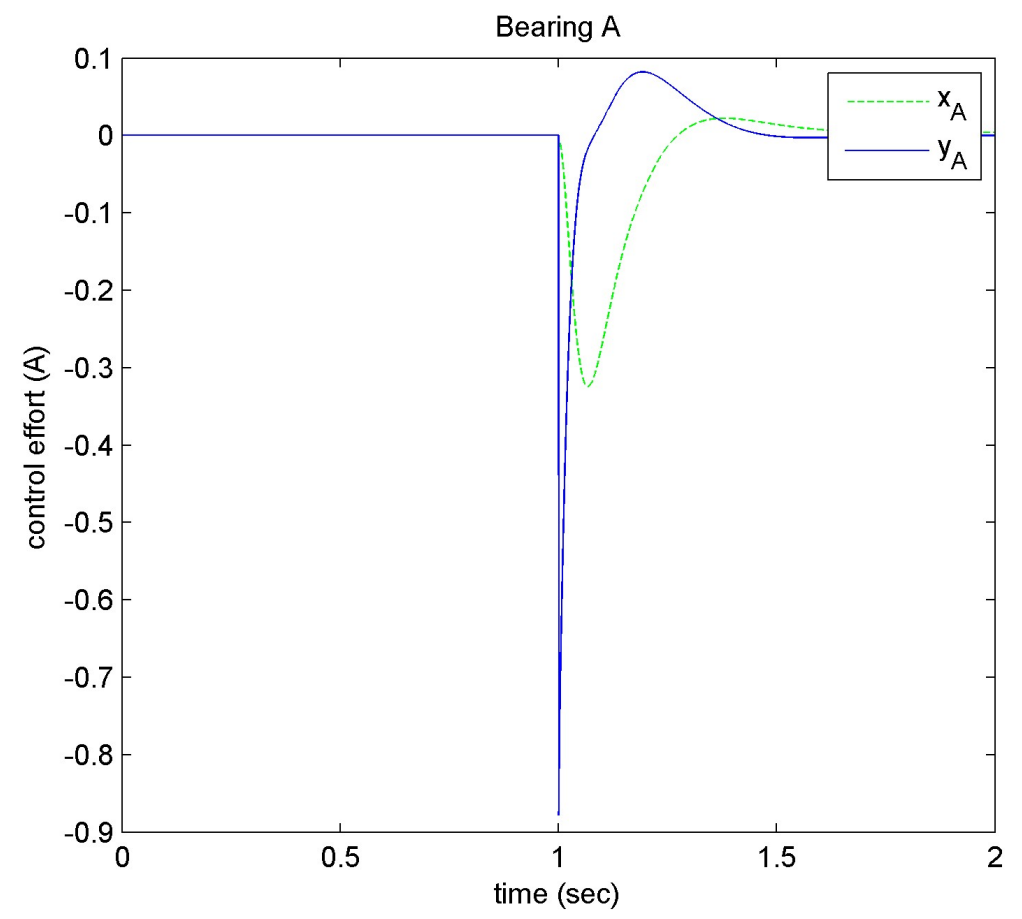

Fig. 36. Coil input currents in amps for the AMB denoted by $A$ using an LQR controller with an integrator. Physically, the $A$ bearing is on the opposite end of the shaft from the impeller.

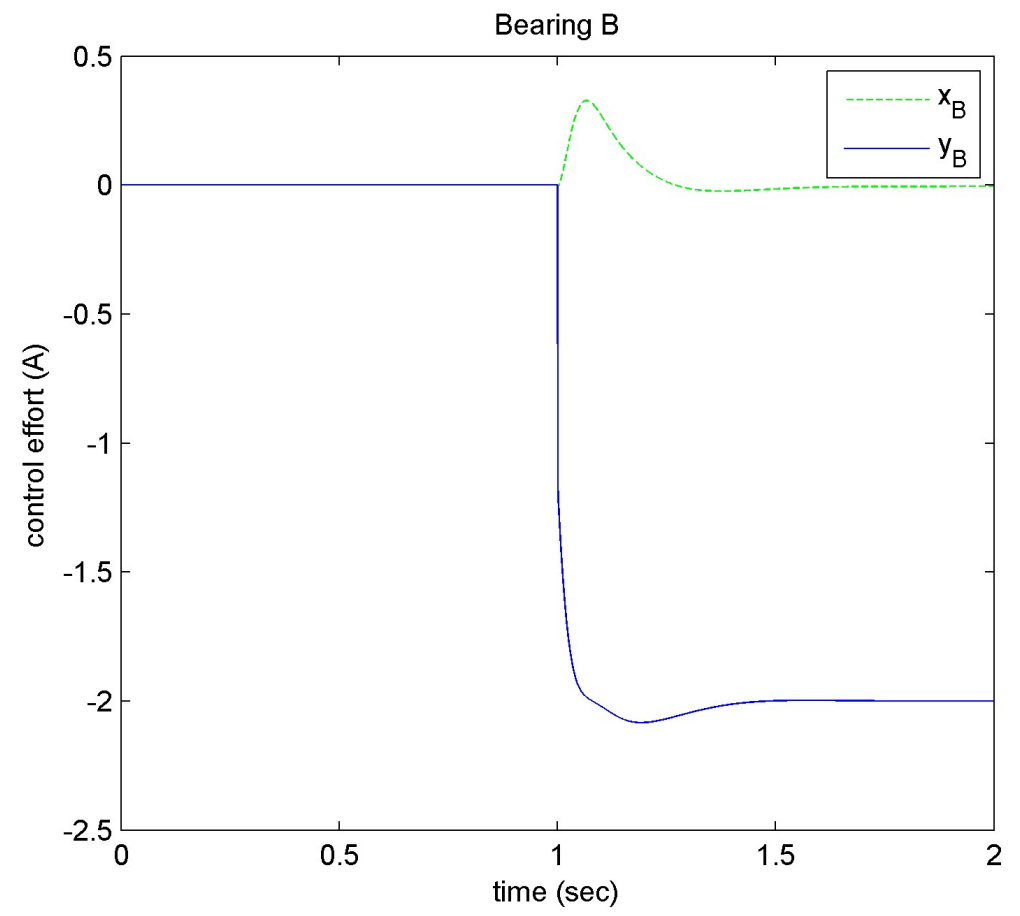

Fig. 37. Coil input currents in amps for the AMB denoted by $B$ using an LQR controller with an integrator. Physically, the $B$ bearing is on the end of the shaft nearest to the impeller. 



\section{OPTIMIZATION AND EMBEDDED DESIGN}

While this section is beyond the scope of the this project, it is closely related to the embedded sensors and controls concept. It illustrates an additional advantage of designing the control system as an embedded system.

An important element in the modeling, simulation, and control system design is understanding the behavior of the system under a variety of circumstances. This becomes more critical when the system operates in extreme environments. These environments make designing functional devices that rely on material properties or other types of classical segmented design techniques difficult or impossible, depending on the environmental factors and the device functionality.

The embedded system design approach where sensors and controls are deeply integrated into the device operation can take advantage of virtualizing the functionality of a device so that it no longer relies solely on the physical properties of the materials or the device geometry to drive operation. As an example, four-stroke engines typically rely on a cam shaft to govern the timing with which the engine valves open and close. Some auto manufacturers are moving to electronically or hydraulically actuated valves so that the valve timing can be controlled by computer. Embedding sensors and control improves the system performance by adding a level of flexibility and responsiveness to the system that is not achievable with a constant geometry cam shaft. Specifically, the system can now be optimized for power or fuel economy over the full range of engine operating conditions and variations in driving conditions in real time.

This ability to optimize performance during operation that is gained from embedding sensor and controls can also have advantages during the design phase. To take advantage of the embedded systems approach, the dynamic behavior of the system must be understood. This is particularly true with the canned rotor pump, which is the test-bed for demonstrating the performance gains possible with embedded system design. It is a highly interconnected system, so designing each subsystem in isolation and then combining them would not yield a workable system. The design of the mechanical and electrical system affects and limits the performance of the control system, and in return, the control system performance bounds the mechanical and electrical design space.

The AMBs, switched reluctance motor, rotordynamics, fluid bearing effects, power electronics, sensors, impeller characteristics, temperature dependent properties, and electrical characteristics all interlink and affect the behavior of the other systems. For example, equations Egs. (66) and (67) both demonstrate the interchange of energy between the magnetic system, the electrical system, and the mechanical system. By looking at the system's dynamic behavior and analyzing the system as a whole, the designer can choose critical parameters of interest and formulate the design process as a series of optimizations.

As an example, the design of the AMB requires defining a few key parameters, specifically $s_{0}$ the nominal air gap, the bearing parameters $k_{i}$ and $k_{s}, N$ the number of coils in a bearing winding, $i_{0}$ the coil current bias, $F_{\text {max }}$ the maximum bearing force desired, and $A$ the stator tooth area.

Initially, some of the bounds on the design variables can be set algebraically due to material constraints. From Eg. (37), with $B_{\max }$ denoting the maximum magnetic field at operating temperature that is a function of the stator lamination material properties, the minimum tooth area $A_{\min }$ can be calculated as

$$
A_{\min }=\frac{F_{\max } \mu_{0}}{B_{\max }^{2}} .
$$


The standard constrained nonlinear optimization problem can be expressed as follows:

$$
\begin{array}{r}
\min _{x} f(x) \quad \text { subject to } \\
c(x) \leq 0, \\
A x \leq b, \\
x_{l b} \leq x \leq x_{u b},
\end{array}
$$

where $f(x)$ is the cost function, $x$ is a vector of design variables, $c(x) \leq 0$ and $A x \leq b$ are the constraints, and $x_{l b}, x_{u b}$ are the lower and upper bounds on the design variables, respectively.

Applied to the design of the magnetic bearing, multiple cost functions can be defined depending upon the performance requirements for the system. In this case, the cost function will be defined to minimize the deflection of the rotor under dynamic loads, i.e.,

$$
\begin{array}{r}
f\left(k_{P}, N, i_{0}, s_{0}\right)=\frac{2 k N^{2} i_{0}}{s_{0}^{2}} k_{P}+\frac{2 k N^{2} i_{0}^{2}}{s_{0}^{3}} \quad \text { s.t. } \\
0 \leq i_{0} \leq i_{\max }, \\
0 \leq s_{0} \leq s_{\max }, \\
0 \leq N \leq N_{\max }, \\
k_{P} \leq 0, \\
\frac{F_{d}}{2 k N^{2}\left(i_{0} s_{0} k_{P}+i_{0}^{2}\right)}-\frac{1}{4 s_{0}^{2}} \leq 0,
\end{array}
$$

where $i_{\max }$ is the maximum desired bias current, $s_{\max }$ is the upper bound on the rotor/stator gap, $N_{\max }$ is the maximum number of turns on the stator windings, which is related to the wire gauge and stator lamination geometry, $k=1 / 2\left(\mu_{0} A\right)$ is a constant, and $F_{d}$ is the maximum expected disturbance force on the system. This function is derived from Eg. (55) and is related to the effective spring rate of the closed-loop system. The last condition guarantees that the maximum rotor movement under the maximum disturbance force is less than one-fourth of the total air gap. Figure 38 shows the cost function when $N=100$. The boundary conditions are not shown in the figure. 


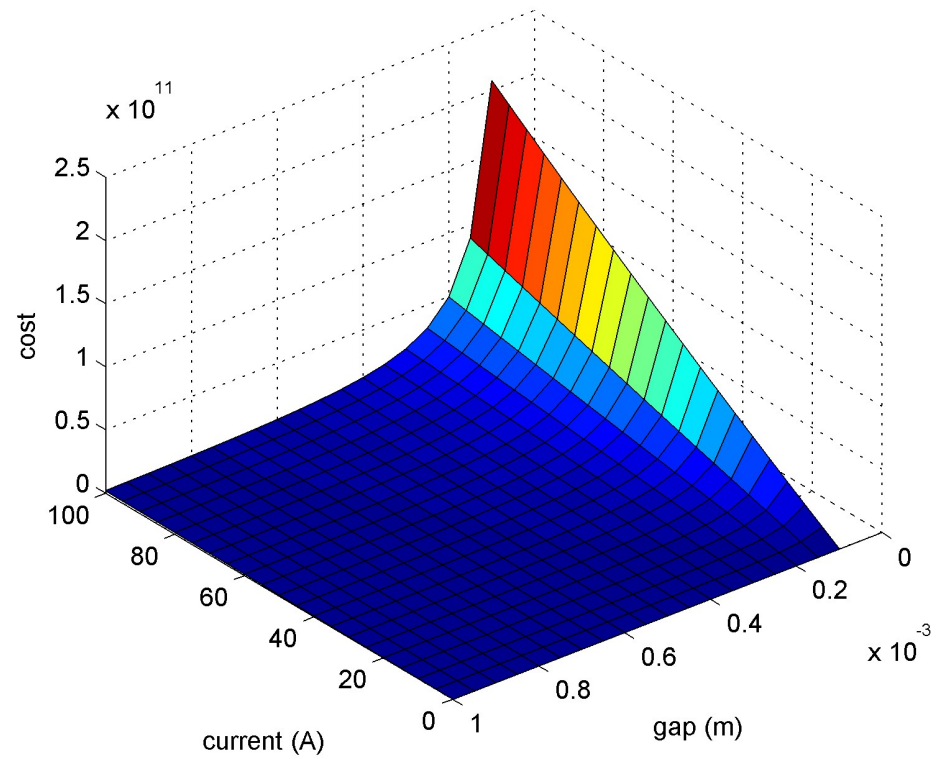

Fig. 38. Cost function to optimize the magnetic bearing design. None of the boundary conditions are included in this figure. 



\section{CONCLUSION AND NEXT STEPS}

In this report, nonlinear models were developed to describe the dynamic behavior of the rotordynamics, fluid forces, rotor can electromagnetic behavior, and AMB dynamics. These models were then used to analytically examine the unforced behavior of the system. The models were then used to design controllers using the industry standard PD controller technique, a PID controller design, a voltage control scheme, and an LQR controller. These models and controllers were validated using simulations in MATLAB $^{\complement}$ and Simulink ${ }^{\complement}$. Design optimization techniques were also formulated for the conceptual pump design that integrate mechanical, electrical, magnetic, and control system design considerations. Optimization techniques such as these allow the embedding of instrumentation and controls to be approached in a manner that balances all the competing design criteria.

The majority of AMBs are operated without fluid in the gap between rotor and stator, and the addition of this fluid bearing effect adds cross-coupling between the rotor modes beyond the gyroscopic coupling. The control system validation shows that the control design techniques employed for the AMBs are sufficient to stabilize the system and that advanced techniques such as LQR control are better able to stabilize the system because they do not assume that each state is decoupled from the other states. Further work on analyzing controller response to rotor imbalance needs to be done. Additionally, analysis of the effects on the system dynamics of the axial bearing forces and non-constant rotor speed should be undertaken.

Now that the theoretical feasibility of the control system design and conceptual pump design has been established using the linear models, design and control system optimization activities can begin and the prototype test-bed design finalized. This activity includes performing a sensitivity analysis of the controller to variations in model parameters such as the protective can thickness. This sensitivity analysis will provide limits on magnetic and controller bandwidth along with a better characterization of controller performance. These limits in turn will be used to establish the feasibility of the measurement techniques to provide sufficient bandwidth and acceptable error. More work is needed to determine requirements for implementation of the control algorithms. These requirements include the computation throughput, calculation precision, measurement sampling, output actuation, etc., needed to perform the measurements, process the algorithm, and provide the desired actuation.

After the prototype test-bed has been built, system model identification and validation activities and comparison of the analytical dynamical models with the experimentally identified dynamical models can begin. Once the models have been validated, the test-bed can be used to develop and compare the performance of advanced embedded instrumentation and controller designs. 



\section{REFERENCES}

1. R. A. Kisner, A. M. Melin, T. A. Burress, D. L. Fugate, D. E. Holcomb, J. B. Wilgen, J. M. Miller, D. F. Wilson, P. C. Silva, L. J. Whitlow, and F. J. Peretz, Embedded sensors and controls to improve component performance and reliability: Conceptual design report, Oak Ridge National Laboratory ORNL/TM-2012/433, (2012).

2. R. A. Kisner, D. L. Fugate, A. M. Melin, D. E. Holcomb, D. F. Wilson, P. C. Silva, and C. C. Molina, Evaluation of manufacturability of embedded sensors and controls with canned rotor pump system, Oak Ridge National Laboratory ORNL/TM-2013/269, (2013).

3. A. Traxler and E. Maslen, Magnetic Bearings: Theory, Design, and Application to Rotating Machinery, Springer, 2010.

4. B. R. Munson, D. F. Young, and T. H. Okiishi, Fundamentals of Fluid Mechanics, John Wiley \& Sons, 1998.

5. M. D. Hersey, Theory and Research in Lubrication, John Wiley \& Sons, 1966.

6. U. Yücel, "Calculation of the dynamic coefficients for fluid film journal bearings," Journal of Engineering Sciences, 11(3), 2005.

7. Vacuumschmelze, "Soft magnetic cobalt-iron-alloys," http://www.vacuumschmelze.com/fileadmin/ docroot/medialib/documents/broschueren/htbrosch/Pht-004_e.pdf, 2001.

8. N. S. Nise, Control Systems Engineering, John Wiley \& Sons, 2000.

9. R. T. Stefani, B. Shahian, C. J. S. Jr., and G. H. Hostetterq, Design of Feedback Control Systems, Oxford University Press, 2002.

10. D. S. Bernstein, Matrix Mathematics, 2nd ed. Princeton University Press, 2009.

11. J. Thoma, Simulation by Bondgraphs, Springer-Verlag, 2009.

12. D. Vischer and H. Bleuler, "A new approach to sensorless and voltage controlled ambs based on network theory concepts," in Proceedings of the Second International Symposium on Magnetic Bearings, Tokyo, Japan, 1990. 